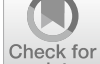

Check for

updates

Cite as

Nano-Micro Lett.

(2021) 13:143

Received: 31 March 2021

Accepted: 11 May 2021

Published online: 14 June 2021

(C) The Author(s) 2021

\section{Applications of 2D-Layered Palladium Diselenide and Its van der Waals Heterostructures in Electronics and Optoelectronics}

\author{
Yanhao Wang ${ }^{1}$, Jinbo Pang ${ }^{7}$, Qilin Cheng ${ }^{7}$, Lin $\operatorname{Han}^{1}{ }^{凶}$, Yufen $\mathrm{Li}^{7}$, Xue Meng ${ }^{1}$, \\ Bergoi Ibarlucea ${ }^{12,13,14,15}$, Hongbin Zhao ${ }^{10}$, Feng Yang ${ }^{9}$, Haiyun Liu ${ }^{7}$, \\ Hong Liu $^{7,8}$, Weijia Zhou ${ }^{7}$, Xiao Wang ${ }^{11}$, Mark H. Rummeli ${ }^{2,3,4,5,6}$, Yu Zhang ${ }^{1} \bowtie$, \\ Gianaurelio Cuniberti ${ }^{12,13,14,15}$
}

\title{
HIGHLIGHTS
}

- The structure-property relationship of $\mathrm{PdSe}_{2}$ is discussed, i.e., layer number vs. tunable bandgap, pentagonal structure vs. anisotropybased polarized light detection.

- The synthesis approaches of $\mathrm{PdSe}_{2}$ are thoroughly compared, including bottom-up methods such as chemical vapor transport for bulk crystals, chemical vapor deposition for thin films and single-crystal domains, selenization of Pd films. Besides, top-down strategies are discussed, covering the mechanical exfoliation of bulk crystals, plasma thinning, and vacuum annealing as well as phase transition.

- The emerging devices of $\mathrm{PdSe}_{2}$ and its van der Waals heterostructures have been delivered such as metal/semiconductor contact, Schottky junction transistors, field-effect transistors, photodetectors, $p-n$ junction-based rectifiers, polarized light detector, and infrared image sensors.

- Future opportunities of $\mathrm{PdSe}_{2}$-based van der Waals heterostructures are given including logic gate-based digital circuits, RF-integrated circuits, Internet of Things, and theoretical calculation as well as big data for materials science.

Yanhao Wang and Jinbo Pang contributed equally to this work.

\inbo Pang, ifc_pangjb@ujn.edu.cn; jinbo.pang@ @otmail.com; Lin Han, hanlin@sdu.edu.cn; Hong Liu, hongliu@sdu.edu.cn; Yu Zhang, yuzhang@sdu.edu.cn

1 Institute of Marine Science and Technology, Shandong University, Qingdao 266237, People's Republic of China

2 College of Energy Soochow Institute for Energy and Materials Innovations, Soochow University, Suzhou 215006, People's Republic of China

3 Key Laboratory of Advanced Carbon Materials and Wearable Energy Technologies of Jiangsu Province, Soochow University, Suzhou 215006, People's Republic of China

${ }^{4}$ Centre of Polymer and Carbon Materials, Polish Academy of Sciences, M. Curie Sklodowskiej 34, 41-819 Zabrze, Poland

5 Institute for Complex Materials, IFW Dresden 20 Helmholtz Strasse, 01069 Dresden, Germany

6 Institute of Environmental Technology VŠB-Technical University of Ostrava, 17. listopadu 15, Ostrava 708 33, Czech Republic

7 Collaborative Innovation Center of Technology and Equipment for Biological Diagnosis and Therapy in Universities of Shandong, Institute for Advanced Interdisciplinary Research (iAIR), University of Jinan, Shandong, Jinan 250022, People's Republic of China

8 State Key Laboratory of Crystal Materials, Center of Bio and Micro/Nano Functional Materials, Shandong University, 27 Shandanan Road, Jinan 250100, People's Republic of China

9 Department of Chemistry, Guangdong Provincial Key Laboratory of Catalytic Chemistry, Southern University of Science and Technology, Shenzhen, Guangdong 518055, People's Republic of China

${ }^{10}$ State Key Laboratory of Advanced Materials for Smart Sensing, GRINM Group Co. Ltd., Xinwai Street 2, Beijing 100088, People's Republic of China

${ }^{11}$ Shenzhen Institutes of Shenzhen Institutes of Advanced Technology, Chinese Academy of Sciences, 1068 Xueyuan Avenue, Shenzhen University Town, Shenzhen 518055, People's Republic of China

${ }^{12}$ Institute for Materials Science and Max Bergmann Center of Biomaterials, Technische Universität Dresden, 01069 Dresden, Germany

${ }^{13}$ Center for Advancing Electronics Dresden, Technische Universität Dresden, 01069 Dresden, Germany

${ }^{14}$ Dresden Center for Computational Materials Science, Technische Universität Dresden, 01062 Dresden, Germany

${ }^{15}$ Dresden Center for Intelligent Materials (GCL DCIM), Technische Universität Dresden, 01062 Dresden, Germany 
ABSTRACT The rapid development of twodimensional (2D) transition-metal dichalcogenides has been possible owing to their special structures and remarkable properties. In particular, palladium diselenide $\left(\mathrm{PdSe}_{2}\right)$ with a novel pentagonal structure and unique physical characteristics have recently attracted extensive research interest. Consequently, tremendous research progress has been achieved regarding the physics, chemistry, and electronics of $\mathrm{PdSe}_{2}$. Accordingly, in this review, we recapitulate and summarize the most recent research on $\mathrm{PdSe}_{2}$, including its structure, properties, synthesis, and applications. First, a mechanical exfoliation method to obtain $\mathrm{PdSe}_{2}$ nanosheets is introduced, and large-area synthesis strategies are explained with respect to chemical vapor deposition and metal selenization. Next, the electronic and optoelectronic properties of $\mathrm{PdSe}_{2}$ and related heterostructures, such as field-effect transistors, photodetectors, sensors, and thermoelec-

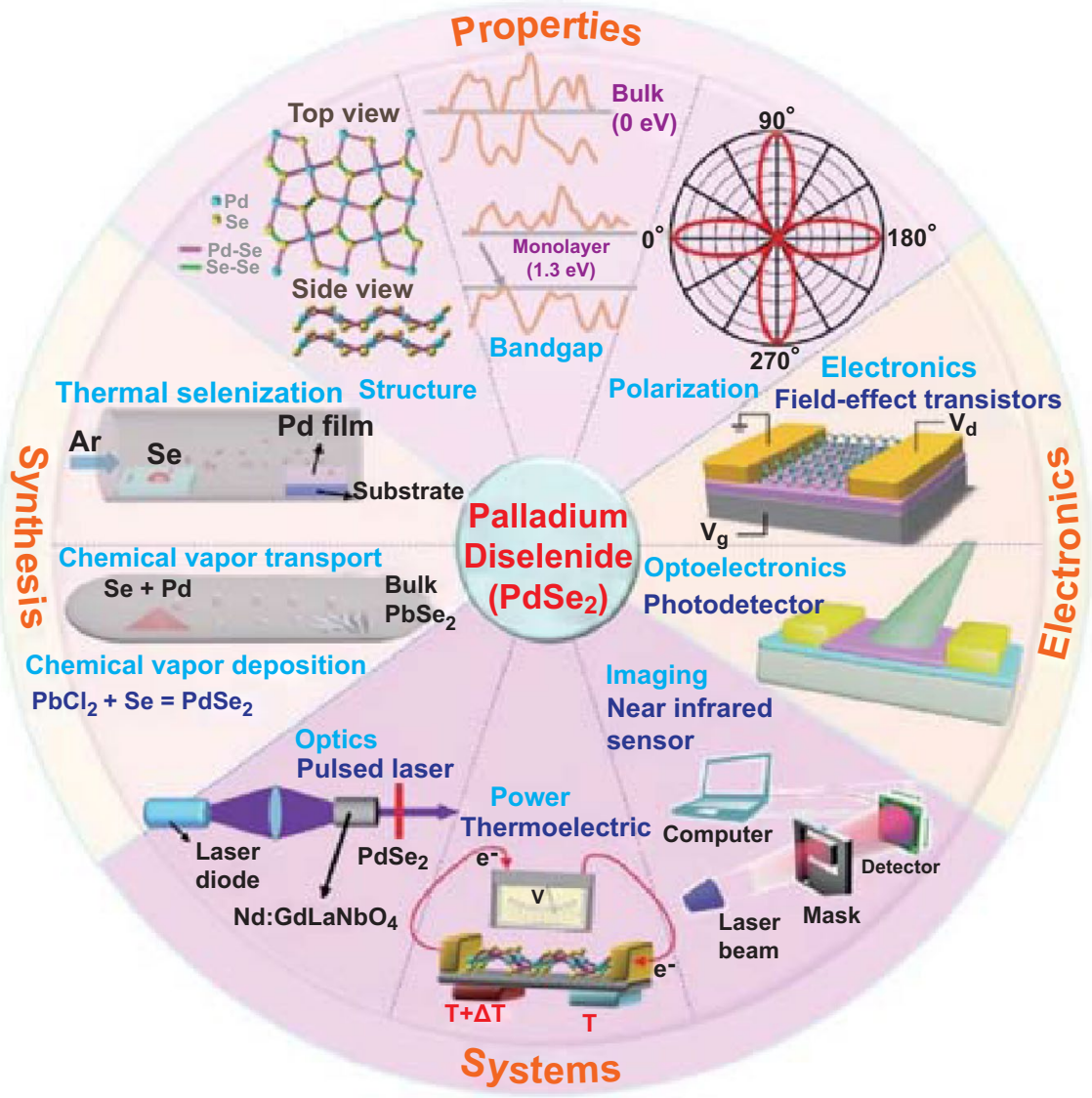
tric devices, are discussed. Subsequently, the integration of systems into infrared image sensors on the basis of $\mathrm{PdSe}_{2}$ van der Waals heterostructures is explored. Finally, future opportunities are highlighted to serve as a general guide for physicists, chemists, materials scientists, and engineers. Therefore, this comprehensive review may shed light on the research conducted by the $2 \mathrm{D}$ material community.

KEYWORDS Palladium diselenide; nTMDC; Synthesis; Field-effect transistors; Photodetectors; Sensors

\section{Introduction}

Significant research has been conducted on two-dimensional (2D) materials, including conductors (graphene) [1], semiconductors $\left(\mathrm{MoS}_{2}\right)$, superconductors $\left(\mathrm{NbSe}_{2}\right)$, and insulators (h-BN). The family of 2D-layered materials, possessing unique structures and extraordinary physical and chemical properties, has been continuously expanded with the addition of members such as transition-metal dichalcogenides (TMDCs) [2], phosphorene, borophene, and MXenes. These 2D materials have been widely employed in biomedical engineering [3], electronics and optoelectronics, photonics, optics, and related devices. Besides, 2D materials have boosted the field of smart sensing such as gas sensors [4].
They exhibit significant potential in devices such as photodetectors and photovoltaic cells; this is attributed to their distinct resonance absorption in the visible to near-infrared spectrum.

The family of TMDCs is an important component of $2 \mathrm{D}$ materials with a general formula of $\mathrm{MX}_{2}$, where $\mathrm{M}$ is a transition element and $\mathrm{X}$ is a chalcogen element. According to the International Union of Pure and Applied Chemistry (IUPAC) [5], transition elements generally comprise those from group 3 to group 12. TMDCs exhibit remarkable properties such as tunable bandgap, stability in air, and good charge transport, which is of great significance to the development of modern technology. Currently, more commonly discussed TMDCs are group-6 TMDCs [6], which 
primarily include $\mathrm{MoS}_{2}, \mathrm{MoSe}_{2}, \mathrm{MoTe}_{2}, \mathrm{WS}_{2}, \mathrm{WSe}_{2}$, and $\mathrm{WTe}_{2}$. Recently, 2D TMDCs and their heterojunction have attracted more and more research interest in the field of broadband photodetectors due to their excellent electronic and optoelectronic properties and show broadband photodetection from UV to IR [7]. In fact, TMDCs have retained significant research value for fundamental physics and device applications.

\subsection{Emerging Noble Transition-Metal Dichalcogenides}

Dichalcogenides of group-10 transition metals $\mathrm{MX}_{2}$ $(\mathrm{M}=\mathrm{Pd}, \mathrm{Pt}, \mathrm{X}=\mathrm{S}, \mathrm{Se}, \mathrm{Te}$ ) have recently received increased research attention owing to their novel properties. They are often referred to as noble transition-metal dichalcogenides (nTMDCs) because all the metal elements in group 10 are noble metals [8]. Here, nTMDCs [9] primarily refer to $\mathrm{PtS}_{2}$, $\mathrm{PdS}_{2}, \mathrm{PtSe}_{2}$, and $\mathrm{PdSe}_{2}$, and they show a significant intrinsic nature resulting from rich $d$-electron content. Besides,
$\mathrm{PtTe}_{2}$-based photodetectors demonstrate an air stable and high performance in MIR photodetection up to $10.6 \mu \mathrm{m}$ [10].

The fundamental properties of the selected nTMDCs are listed in Table 1 . The nTMDCs are, however, yet to be fully understood; therefore, there is much scope for research in this area.

Before introducing the $\mathrm{PdSe}_{2}$, we first look at the properties of other nTMDCs. $\mathrm{PtS}_{2}$ exhibits very strong interlayer interactions and layer-dependent indirect bandgaps ranging from 1.6 (monolayer) to 0.25 (bulk) $\mathrm{eV}$. In recent years, few-layer $\mathrm{PtS}_{2}$ has become a promising material for field-effect transistors (FETs) with high mobility and on/ off ratios. Furthermore, $\mathrm{PtS}_{2}$-based devices have demonstrated excellent performance with respect to photodetection and sensing. Similarly, 2D PtSe ${ }_{2}$ shows prominent layer-dependent properties, and the bandgap of monolayer $\mathrm{PtSe}_{2}$ is $1.2 \mathrm{eV}$, while that of bulk $\mathrm{PtSe}_{2}$ is zero. The carrier mobility of few-layer $\mathrm{PtSe}_{2}$ can theoretically exceed $10^{3} \mathrm{~cm}^{2} \mathrm{~V}^{-1} \mathrm{~s}^{-1}$, and very high stability in air is demonstrated [11]. Few-layered $\mathrm{PtSe}_{2}$ has been utilized in a

Table 1 The basic properties of the noble transition-metal dichalcogenides group

\begin{tabular}{|c|c|c|c|c|c|c|c|}
\hline Material types & Phase & Bandgap & Lattice parameters & Lattice structure & Crystal system & Space group & $\begin{array}{l}\text { Refer- } \\
\text { ences }\end{array}$ \\
\hline $\mathrm{PdSe}_{2}$ & Marcasite & $\begin{array}{l}0 \mathrm{eV} \text { (bulk) } \\
1.33 \mathrm{eV}(1 \mathrm{~L})\end{array}$ & $a=5.74 \AA ; b=5.92 \AA ; c=7.69 \AA$ & Pentagonal & Orthorhombic & Pbca [61] & {$[35]$} \\
\hline $\mathrm{PdSe}_{2}$ & Marcasite & & $a=5.79 \AA ; b=5.95 \AA ; c=8.59 \AA$ & Pentagonal & Orthorhombic & Pbca [61] & [132] \\
\hline $\mathrm{PdSe}_{2}$ & $1 \mathrm{~T}$ & $\begin{array}{l}0 \mathrm{eV}(\geq 2 \mathrm{~L}) \\
0.778 \mathrm{eV}(1 \mathrm{~L})\end{array}$ & $a=3.73 \AA ; c=4.79 \AA$ & n.a & Hexagonal & & [133] \\
\hline $\mathrm{PdSe}_{2}$ & $2 \mathrm{H}$ & n.a & $a=3.58 \AA ; c=10.90 \AA$ & n.a & Hexagonal & & [133] \\
\hline $\mathrm{PdSe}_{2}$ & Pyrite & n.a & $\mathrm{a}=5.74 \AA ; b=5.86 \AA ; c=7.53 \AA$ & n.a & Orthorhombic & & {$[133]$} \\
\hline $\mathrm{PdSe}_{2}$ & Marcasite & n.a & $a=5.06 \AA ; b=6.12 \AA ; c=3.89 \AA$ & n.a & Orthorhombic & & [133] \\
\hline $\mathrm{PdS}_{2}$ & Marcasite & & $a=5.50 \AA ; b=5.59 \AA ; c=8.61 \AA$ & pentagonal & Orthorhombic & Pbca [61] & [134] \\
\hline $\mathrm{PdS}_{2}$ & $1 \mathrm{~T}$ & $\begin{array}{l}0 \mathrm{eV}(\geq 2 \mathrm{~L}) \\
1.1 \mathrm{eV}(1 \mathrm{~L})\end{array}$ & $a=3.068 \AA$ & n.a & Hexagonal & Pbca [61] & [14] \\
\hline $\mathrm{PdS}_{2}$ & $2 \mathrm{H}$ & n.a & $a=3.82 \AA ; c=9.33 \AA$ & n.a & Hexagonal & n.a & [133] \\
\hline $\mathrm{PdS}_{2}$ & Pyrite & $\begin{array}{l}0 \mathrm{eV} \text { (Bulk) } \\
1.399 \mathrm{eV}(1 \mathrm{~L})\end{array}$ & $a=5.45 \AA ; b=5.53 \AA ; c=7.20 \AA$ & n.a & Orthorhombic & n.a & {$[133]$} \\
\hline $\mathrm{PdS}_{2}$ & Marcasite & n.a & $a=4.78 \AA ; b=5.67 \AA ; c=3.79 \AA$ & n.a & Orthorhombic & n.a & [133] \\
\hline $\mathrm{PdTe}_{2}$ & Merenskyite & n.a & n.a & n.a & Trigonal & P-3m1 [164] & [135] \\
\hline $\mathrm{PdTe}_{2}$ & $1 \mathrm{~T}$ & n.a & $a=b=4.0365 \AA ̊ \AA c=5.1262 \AA$ & n.a & Hexagonal & & [136] \\
\hline $\mathrm{PdTe}_{2}$ & $2 \mathrm{H}$ & n.a & $a=3.83 \AA \circ ; c=11.60 \AA$ & n.a & Hexagonal & & [133] \\
\hline $\mathrm{PdTe}_{2}$ & Pyrite & $0 \mathrm{eV}(\geq 1 \mathrm{~L})$ & $a=b=c=6.54 \AA$ & n.a & Orthorhombic & & [133] \\
\hline $\mathrm{PdTe}_{2}$ & Marcasite & $\mathrm{n} . \mathrm{a}$ & $a=5.40 \AA ; b=6.65 \AA ; c=4.10 \AA$ & n.a & Orthorhombic & & [133] \\
\hline $\mathrm{PtSe}_{2}$ & $1 \mathrm{~T}$ & $\begin{array}{l}0 \mathrm{eV} \text { (bulk) } \\
1.17 \mathrm{eV}(1 \mathrm{~L})\end{array}$ & $a=b=3.73 \AA ; c=5.08 \AA$ & Octahedral crystal & Hexagonal & & [11] \\
\hline $\mathrm{PtS}_{2}$ & $1 \mathrm{~T}$ & $\begin{array}{l}0.25 \mathrm{eV} \text { (bulk) } \\
1.6 \mathrm{eV}(1 \mathrm{~L})\end{array}$ & $a=b=3.54 \AA ; c=5.04 \AA$ & $\begin{array}{l}\text { Octahedral } \\
\text { coordination } \\
\text { structure }\end{array}$ & Hexagonal & & [30] \\
\hline
\end{tabular}


variety of applications, such as FETs and photodetectors. $\mathrm{PtSe}_{2}$ shows good potential in piezoelectric devices, saturable absorbers, and electrochemical energy conversion. The structure of $\mathrm{PdS}_{2}$ comprises a pentagonal network, which includes two Pd atoms and three $\mathrm{S}$ atoms distributed on the atomic plane [12]. Monolayer $\mathrm{PdS}_{2}$ has two stable structures: one is a standard $1 \mathrm{~T}$ structure and the other involves a bulk-like geometry [13]. Through predictions and calculations, monolayer $\mathrm{PdS}_{2}$ has been determined to possess a semiconducting feature with a bandgap of approximately $1.1 \mathrm{eV}$, while bilayer $\mathrm{PdS}_{2}$ possesses a semimetallic feature [14]. Through first-principle calculations, a few-layer $\mathrm{PdS}_{2}$ has been predicted theoretically with good electronic and optoelectronic properties. However, few experimental synthesis studies have been reported in this regard. Thus far, there remain good opportunities for the material optimization and device applications of
$\mathrm{PdS}_{2}$. But $\mathrm{PdS}_{2}$ pentagonal structure is not thermodynamically stable, which limits its applications. Hence, $\mathrm{PdSe}_{2}$ becomes of importance for exploiting the polarization properties and related optoelectronic applications.

\subsection{Importance of $\mathrm{PdSe}_{2}$}

$\mathrm{PdSe}_{2}$ exhibits unique physical properties such as high carrier mobility, tunable bandgaps, and magnetic transport. $\mathrm{PdSe}_{2}$ has become a popular 2D material owing to its good stability [15], layer-dependent bandgap, and in-plane optical anisotropy [16]. $\mathrm{PdSe}_{2}$ (Scheme 1) has been integrated into electronic [17], thermoelectric, optical [18], and optoelectronic devices [19]. The diverse polymorphisms of $\mathrm{PdSe}_{2}$ provide the platform for investigating the topological states and the applications of quantum information devices [20].

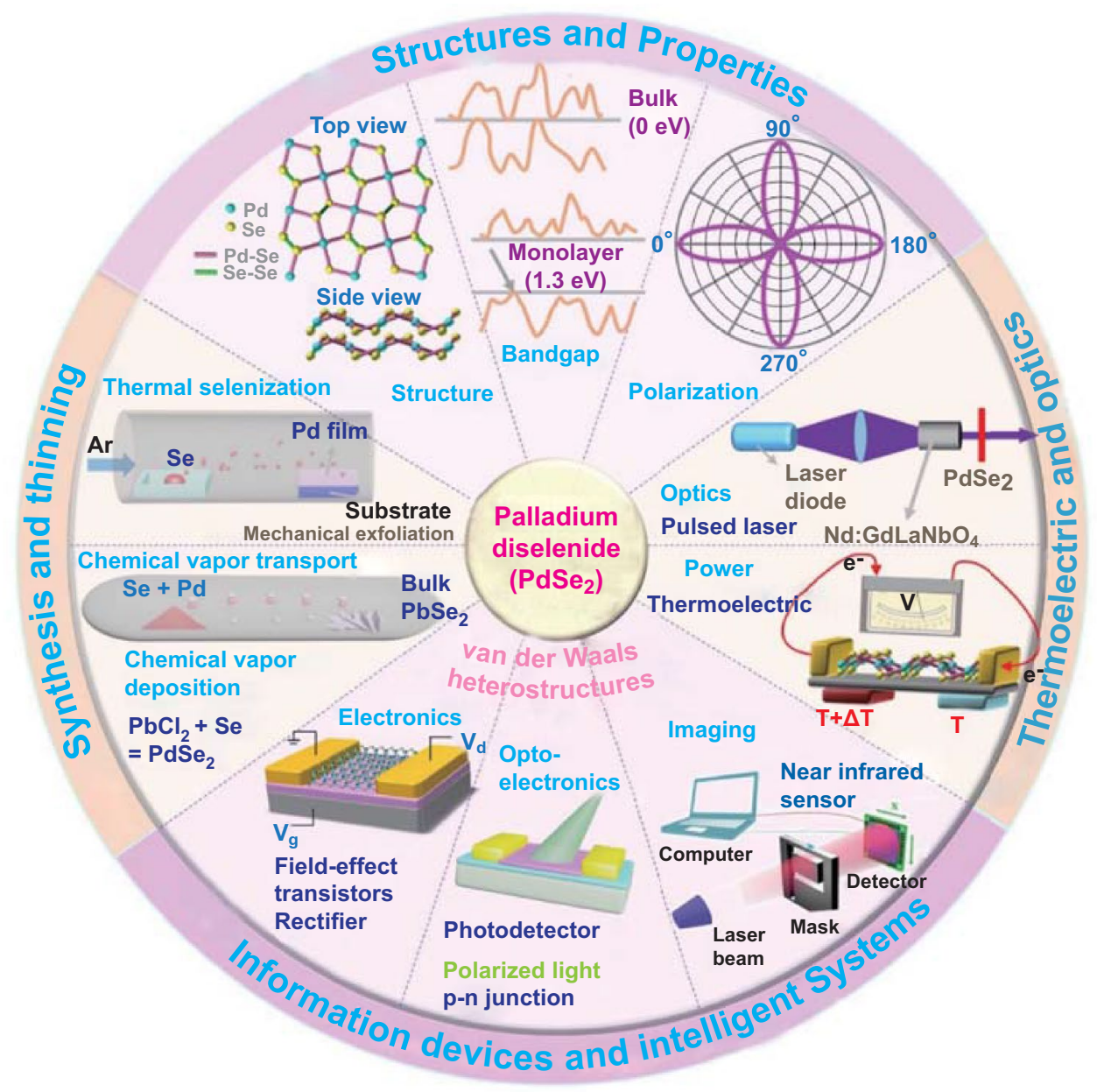

Scheme $1 \mathrm{PdSe}_{2}$ and its heterostructures for electronic, optic, and optoelectronic devices and systems 
$\mathrm{PdSe}_{2}$-based van der Waals heterostructures (vdWHs) have been widely incorporated in current rectifier, polarized light photodetector, and infrared image sensor applications. First, the direct synthesis of $\mathrm{PdSe}_{2}$-based vdWHs has been investigated via deposition of $\mathrm{PdSe}_{2}$ over other 2D materials such as graphene [21], $\mathrm{MoS}_{2}$ [22], $\mathrm{MoSe}_{2}$ [23], GeSe [24], and $\mathrm{SnSe}_{2}$. The stacking with arrayed nanomaterials gives rise to heterostructure devices such as $\mathrm{ZnO}$ nanorods and Si nanowires [25]. A perovskite [26] heterostructure can be formed with $\mathrm{PdSe}_{2}$ using a self-powered image sensor.

In this review, we discuss the most recent developments with regard to $\mathrm{PdSe}_{2}$ and its vdWHs, including approaches for its synthesis and its application in electronics, optoelectronics, and optics. We believe that this comprehensive contribution may attract the attention of research communities as well as industrial engineers interested in $\mathrm{PdSe}_{2}$ material development and device integration.

\section{Structure and Properties of $\mathrm{PdSe}_{2}$}

This section introduces in detail the crystalline structure, electronic structure, energy band, vibrational phonon modes, and phase transition of $\mathrm{PdSe}_{2}$, which are the bases of its application in various fields.

\subsection{Crystal Structure}

As a 2D-puckered pentagonal material, $\mathrm{PdSe}_{2}$ possesses orthorhombic lattices and a low symmetry, and it was identified as the first TMDC with a pentagonal structure [27]. The crystalline structure of $\mathrm{PdSe}_{2}$ has been studied from as early as 1952 [28], owing to which a good foundation for current research has been laid. Most recently, 2D materials with pentagonal structures have attracted much research attention. Examples include penta-graphene, penta- $\mathrm{PdS}_{2}$ [12], penta$\mathrm{SnS}_{2}$, penta-silicene, and penta-germanene. The structures of these pentagonal materials differ from most hexagonal structures in 2D materials with high symmetry. They can still possess a relatively low symmetry in regular corrugated modes. Therefore, unique physical properties emerge with pentagonal structures, leading to novel electronic applications.

Figure 1a shows the top and side views of the monolayer $\mathrm{PdSe}_{2}$ structure; it can be clearly seen that the one-unit cell contains four Pd atoms and eight Se atoms (top plane). In one $\mathrm{PdSe}_{2}$ layer, the two Se atoms cross the Pd layer in the form of a Se-Se dumbbell (bottom plane).

The uncommon layered structure is composed entirely of pentagonal rings, in which each Pd atom binds to four Se atoms, and two adjacent Se atoms form a covalent bond in one layer [23]. Hence, there exists no dangling bond in one $\mathrm{PdSe}_{2}$ layer, and these layers interact via van der Waals forces, resulting in excellent stability in air. The lattice parameters $a, b$, and $c$ are, respectively, 5.75, 5.87, and $7.69 \AA$ for $\mathrm{PdSe}_{2}$. Each layer of $\mathrm{PdSe}_{2}$ crystal has a vertical puckering height of $1.6 \AA$, where Pd atoms exhibit an unusual planar tetra-coordination [15].

Figure $1 \mathrm{~b}$ shows the corresponding three-dimensional (3D) schematic of a monolayer $\mathrm{PdSe}_{2}$ structure from a projected top view and side view [15], which is similar to that of black phosphorus (BP).

Figure $1 \mathrm{c}, \mathrm{d}$ exhibits the annular dark-field (ADF) image of the $\mathrm{PdSe}_{2}$ crystals, as generated via scanning transmission electron microscopy (STEM), as well as the corresponding image simulations (Fig. 1e, f) [15]. This approach can well prevent the formation of the disordered region of $\mathrm{PdSe}_{2}$ flakes due to the transfer process onto the TEM grid. As can be seen, owing to the difference in symmetry, the even and odd layers of $\mathrm{PdSe}_{2}$ flakes can give rise to a variation in the ADF images. Nonetheless, these patterns are in good agreement with the corresponding image simulations [15]. Moreover, the STEM images verify the puckered structure with waved Pd-Se layers of $\mathrm{PdSe}_{2}$.

The morphology and structure of $\mathrm{PdSe}_{2}$ have shown satisfactory property-structure correlation. Indeed, the anisotropic orientation of the $\mathrm{PdSe}_{2}$ domains results in polarized light detection [29]. The strain engineering influences the phonon response, which demonstrates its potential in the field of flexible electronics. Defect engineering such as vacancies could affect the air stability of the $\mathrm{PdSe}_{2}$ transistor as well as the Ohmic contact. The phase transition mechanism should be investigated for a better understanding, and more new phases of $\mathrm{PdSe}_{2}$ can be exploited for further applications. The high-pressure induced phase of $\mathrm{PdSe}_{2}$ renders a photovoltaic material. The hexagonal $\mathrm{T}$ phase of $\mathrm{PdSe}_{2}$ resulted in a high-efficiency solar cell. The pyrite phase $\mathrm{PdSe}_{2}$ exhibits superconductivity induced by high pressure.

Bulk $\mathrm{PdSe}_{2}$ crystals display $\mathrm{D}_{2 \mathrm{~h}}$ point group symmetry and Pbca space group symmetry [28]. The pentagonal $\mathrm{PdSe}_{2}$ belongs to the phase of marcasite in the crystal system of 
(a)
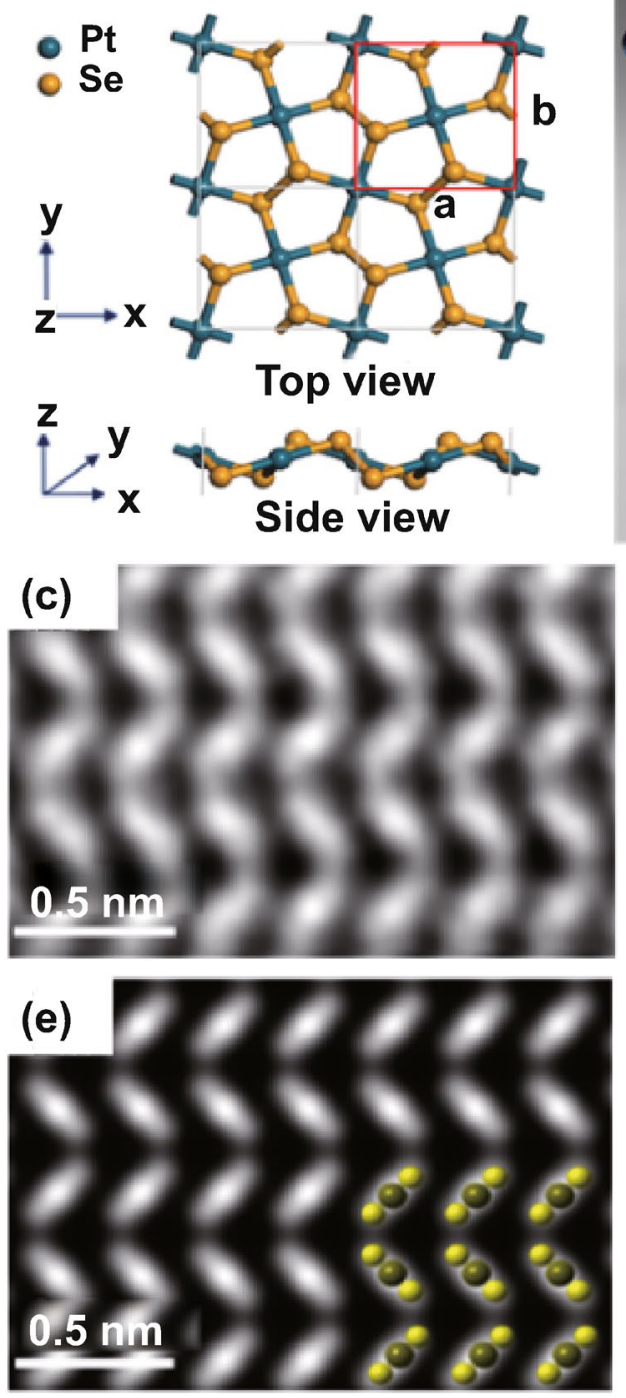

(b)
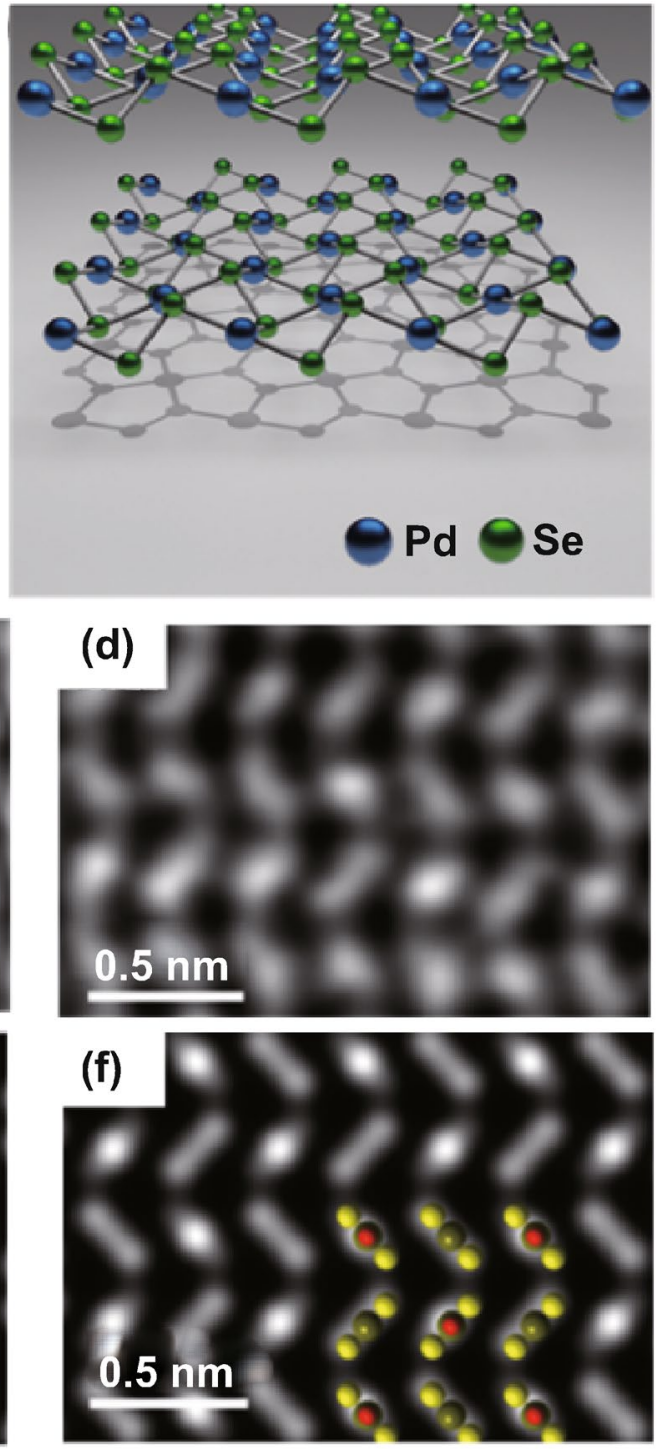

Fig. 1 Atomic structure of $\mathrm{PdSe}_{2}$. a Top view and side view of penta-PdSe $e_{2}$ monolayers, where a unit cell is marked using a red line. The blue and yellow spheres represent the Pd and Se atoms, respectively. Reprinted with permission from Ref. [199]. Copyright 2015, Royal Society of Chemistry. b 3D crystallographic structure of puckered pentagonal PdSe ${ }_{2}$. c, d Z-contrast STEM images of PdSe $\mathrm{P}_{2}$ crystal structure with even and odd numbers of layers. $\mathbf{e}, \mathbf{f}$ Corresponding simulated images of $\mathrm{PdSe}_{2}$ crystals with even and odd numbers of layers. Insets in $\mathbf{e}, \mathbf{f}$ display atomic models of the corresponding STEM images. Reprinted with permission from Ref .[15]. Copyright 2017, American Chemistry Society

orthorhombic [27]. By comparison, thin $\mathrm{PdSe}_{2}$ flakes with an odd number of layers are allocated to space group $P 2{ }_{1} / c$ (No. 14) and point group $C_{2 h}(2 / m)$, which possess inversion symmetry, while thin $\mathrm{PdSe}_{2}$ flakes with an even number of layers are allocated to space group $P c a 2_{1}$ (No. 29) and point group $\mathrm{C}_{2 v}(\mathrm{~mm} 2)$, which do not possess inversion symmetry [15].

\subsection{Electron Orbital Properties}

The conventional hexagonal structures are featured with isotropy, e.g., $\mathrm{MoS}_{2}$. The symmetrical hexagons lead to weak interlayer interaction due to the $\mathrm{d}^{4} s p$ hybridization in TMDCs [20]. Here, the Mo and W elements are in lack of $d$ orbital electrons. Besides, the $d$ orbital of Pt atom and 
$\mathrm{p}_{\mathrm{z}}$ orbital of $\mathrm{S}$ atom are hybridized into $\mathrm{d}^{2} s p^{3}$ type, which accounts for the strong interlayer interaction in $\mathrm{PtS}_{2}$ [30].

But the hybridization between Pd and Se orbitals is complicated in $\mathrm{PdSe}_{2}$. First, one need to understand the electron configuration of these two elements. The Pd metal has a fully occupied $d$ orbital with electron configuration of $[\mathrm{Kr}] 4 \mathrm{~d}^{10}$. And the Se is a $p$-block element, with an electron configuration of $[\mathrm{Ar}] 3 \mathrm{~d}^{10} 4 \mathrm{~s}^{2} 4 \mathrm{p}^{4}$. In a single-layer $\mathrm{PdSe}_{2}$, one $\mathrm{Pd}$ atom is coordinated to four $\mathrm{Se}$ atoms, forming a square-planar structure [31]. Quite often, the $\mathrm{Pd}^{2+}$ results in the $\mathrm{d}^{8}$ configuration such as $\mathrm{PdCl}_{2}$. Therefore, the $\mathrm{PdSe}_{2}$ possesses a phase of marcasite analogous to the $\mathrm{FeS}_{2}$ [27]. The weak hybridization occurs between the $4 \mathrm{~d}_{\mathrm{z}}^{2}$ orbitals of Pd atom and $4 \mathrm{p}_{\mathrm{z}} / 3 \mathrm{~d}_{\mathrm{z}}{ }^{2}$ orbitals of Se atom, which led to the low symmetry [31].

The hybridization of $\mathrm{Pd} 4 \mathrm{~d}$ orbit and $\mathrm{Se} 4 \mathrm{p}$ orbit has resulted in the covalent bond in $\mathrm{PdSe}_{2}$ [32]. The bands near Fermi level are contributed by the $p$ orbitals of Se element. The conductance band minimum and valence band maximum of monolayer $\mathrm{PdSe}_{2}$ have stemmed from the $p$ states of Se and $d$ states of Pd. The spin-orbital coupling does not influence the electronic structure of monolayer $\mathrm{PdSe}_{2}$ [33]. But, with increasing the layer number, the interlayer coupling becomes strong and decreases the bandgap of bilayer and trilayer $\mathrm{PdSe}_{2}$ compared with monolayer $\mathrm{PdSe}_{2}$ [32]. Besides, the stacking types determine the bandgap of $\mathrm{PdSe}_{2}$, e.g., the $\mathrm{AA}$ and $\mathrm{AB}$ stacking for bilayer $\mathrm{PdSe}_{2}$ and the $\mathrm{AAA}, \mathrm{ABA}$, and $\mathrm{ABB}$ stacking for trilayer $\mathrm{PdSe}_{2}$ [32].

Indeed, the pentagonal $\mathrm{PdSe}_{2}$ is analogous to other puckered 2D materials, i.e., phosphorene and silicene, which feature with anisotropy [15]. The buckling of puckered 2D materials lead to a strong spin-orbital coupling between adjacent two layers, which is accounted for the topological quantum phase transition.

With the doping of transition-metal atoms such as $\mathrm{Cr}$ and $\mathrm{Mn}$, new energy levels were introduced into the band structure of $\mathrm{PdSe}_{2}$ [34], which decrease its bandgap and introduce new spin nondegenerate states. These spin states around the Fermi level could cause the spin polarization.

After knowing the electron orbital theory, we now come to discuss the band structure of $\mathrm{PdSe}_{2}$.

\subsection{Electronic Band Structure}

This section discusses the electronic energy band structures and density of states (DOSs) of $\mathrm{PdSe}_{2}$. Similar to that of most layered TMDCs, the indirect bandgap of $\mathrm{PdSe}_{2}$ largely depends on the number of layers.

The bandgap of $\mathrm{PdSe}_{2}$ has been calculated [33] via the approaches of generalized gradient approximation (GGA), density functional theory (DFT) of Perdew, Burke, and Ernzerhof (PBE). Here, the bandgap of $\mathrm{PdSe}_{2}$ is defined as the energy difference between the valence band (VB) and the conduction band (CB). The indirect bandgap of monolayer $\mathrm{PdSe}_{2}$ with semiconducting characteristics is $1.33 \mathrm{eV}$ (Fig. 2a), and this decreases with the increase in the number of $\mathrm{PdSe}_{2}$ layers until the bulk $\mathrm{PdSe}_{2}$ has no bandgap $(0 \mathrm{eV})$ with semimetallic characteristics (Fig. 2d).

In the cases of TMDCs and phosphorene, the valence band maximum (VBM) and conduction band minimum (CBM) are located along the high-symmetry lines. However, in the electronic structure of $\mathrm{PdSe}_{2}$ [35], VBM is located between the high-symmetry $\Gamma$ and $X$, while the CBM is located between $M$ and $\Gamma$ (Fig. 2a).

Meanwhile, the effects of strain, particularly biaxial strains, have been investigated on the electronic and optical properties of $\mathrm{PdSe}_{2}$ [36]. Figure 2b, c shows the evolution of the monolayer $\mathrm{PdSe}_{2}$ energy bands under compressive and tensile strains, respectively. The black line represents the energy band of $\mathrm{PdSe}_{2}$ in the unstrained state, while the other colors represent the energy bands of $\mathrm{PdSe}_{2}$ in the various strained states. The compressive and tensile strains decrease the CBM and increase the VBM of monolayer $\mathrm{PdSe}_{2}$, and the VBM and CBM rise to a maximum value for compressive or tensile strains of $-10 \%$, leading to the minimum bandgap of monolayer $\mathrm{PdSe}_{2}$ [35]. Moreover, under compressive strain along the $x$-direction, the monolayer $\mathrm{PdSe}_{2}$ shows a negative Poisson's ratio, possibly resulting from the Se-Se bond [37].

Figure $2 \mathrm{~d}$ shows the energy band of bulk $\mathrm{PdSe}_{2}$, where the electronic structure shows a negative indirect bandgap with semimetallic characteristics at the DFT level. However, VB and CB are not entangled around the Fermi level [33]. A semimetallic feature of bulk $\mathrm{PdSe}_{2}$ can be observed through 

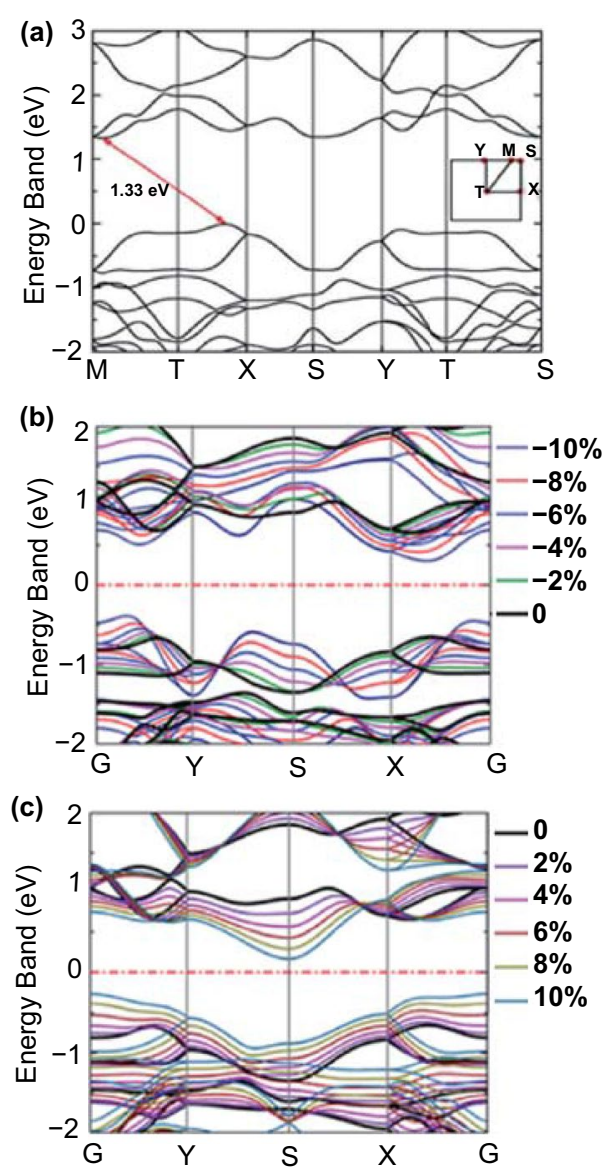
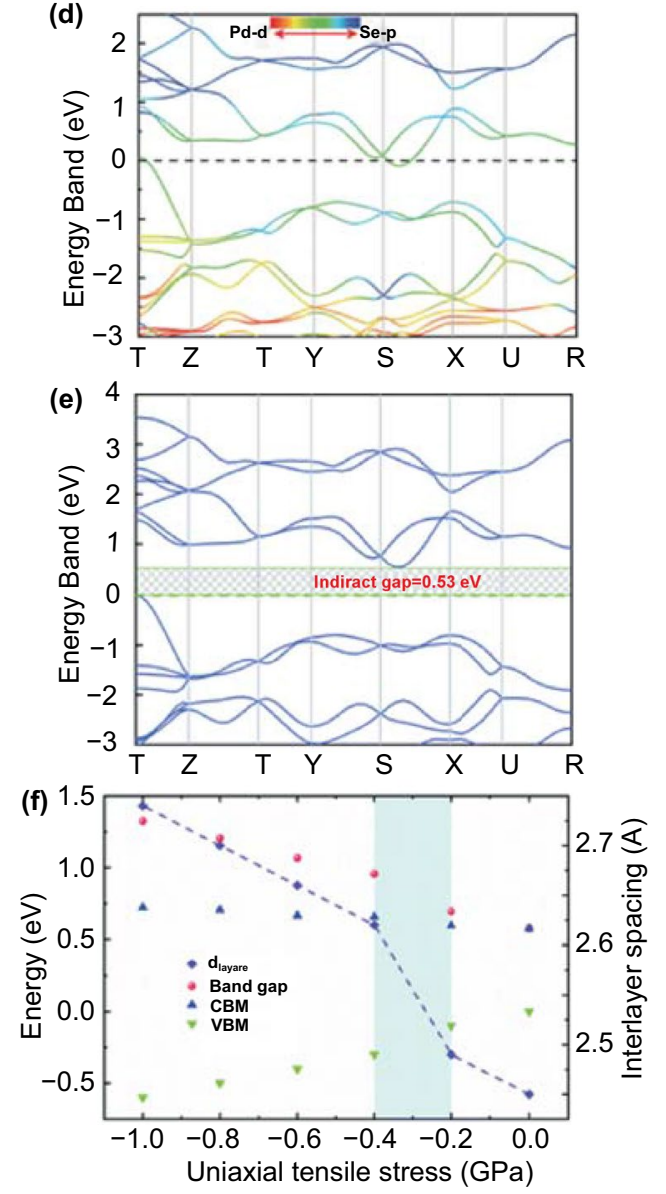

Fig. 2 a Electronic band structure of monolayer $\mathrm{PdSe}_{2}$ with no strain. Reprinted with permission from Ref. [35]. Copyright 2018, Royal Society of Chemistry. Electronic band structure of monolayer $\mathrm{PdSe}_{2}$ with symmetrical biaxial $\mathbf{b}$ compressive, and $\mathbf{c}$ tensile strains. Reprinted with permission from Ref. [36]. Copyright 2018, American Chemistry Society. d Electronic band structure of bulk PdSe ${ }_{2}$, where the Fermi level is set to zero. The red and blue regions represent the contributions from $\mathrm{Pd} 4 \mathrm{~d}$ and $\mathrm{Se} 4 \mathrm{p}$ states, respectively. e Electronic band structure of bulk $\mathrm{PdSe}_{2}$ under a tensile stress of $1.0 \mathrm{GPa}$. f Bandgap, CBM, VBM, and interlayer spacing $\left(\mathrm{d}_{\text {layers }}\right.$ ) of bulk PdSe $\mathrm{P}_{2}$ as a function of the uniaxial tensile stress, where the blue region presents the rapid increase of $d_{\text {layers }}$. Reprinted with permission from Ref. [33]. Copyright 2019, Royal Society of Chemistry

ultraviolet photoemission spectroscopy [26] and optical absorption [25]. However, bulk $\mathrm{PdSe}_{2}$ exhibits semiconducting characteristics from resistivity experiments [38]. Hence, further research is necessary to understand the bandgap of bulk $\mathrm{PdSe}_{2}$ better owing to this contradiction.

Figure 2e reveals the electronic band structure of bulk $\mathrm{PdSe}_{2}$ calculated via DFT under the tensile stress of -1.0 $\mathrm{GPa}$, whereby a bandgap of $0.48 \mathrm{eV}$ is observed. When uniaxial tensile stress is applied to bulk $\mathrm{PdSe}_{2}$ along the outof-plane direction, the lattice parameter $c$ and interlayer distance increase [33]. In orthorhombic $\mathrm{PdSe}_{2}$, the bandgap is positively correlated with the interlayer distance, indicating that the interlayer interaction has a significant influence on the electronic structure. Figure $2 \mathrm{f}$ shows the interlayer spacing $\left(\mathrm{d}_{\text {layers }}\right)$ and bandgap of bulk $\mathrm{PdSe}_{2}$ as a function of the uniaxial tensile stress. As the interlayer spacing increases, VBM decreases dramatically, while CBM increases slightly, resulting in an increase in the bandgap of bulk $\mathrm{PdSe}_{2}$.

Figure 3 depicts the electronic DOSs for both bulk and monolayer $\mathrm{PdSe}_{2}$ calculated in denser $k$ meshes with values of $23 \times 23 \times 17$ and $40 \times 40 \times 1$, respectively [27]. In the inset of Fig. 3a, the bandgap of bulk $\mathrm{PdSe}_{2}$ is $0.03 \mathrm{eV}$, while that of monolayer $\mathrm{PdSe}_{2}$ is approximately $1.43 \mathrm{eV}$ (Fig. 3b). These values are slightly higher than the bandgap values obtained through the traditional GGA-PBE functional, indicating an underestimation of the bandgap 

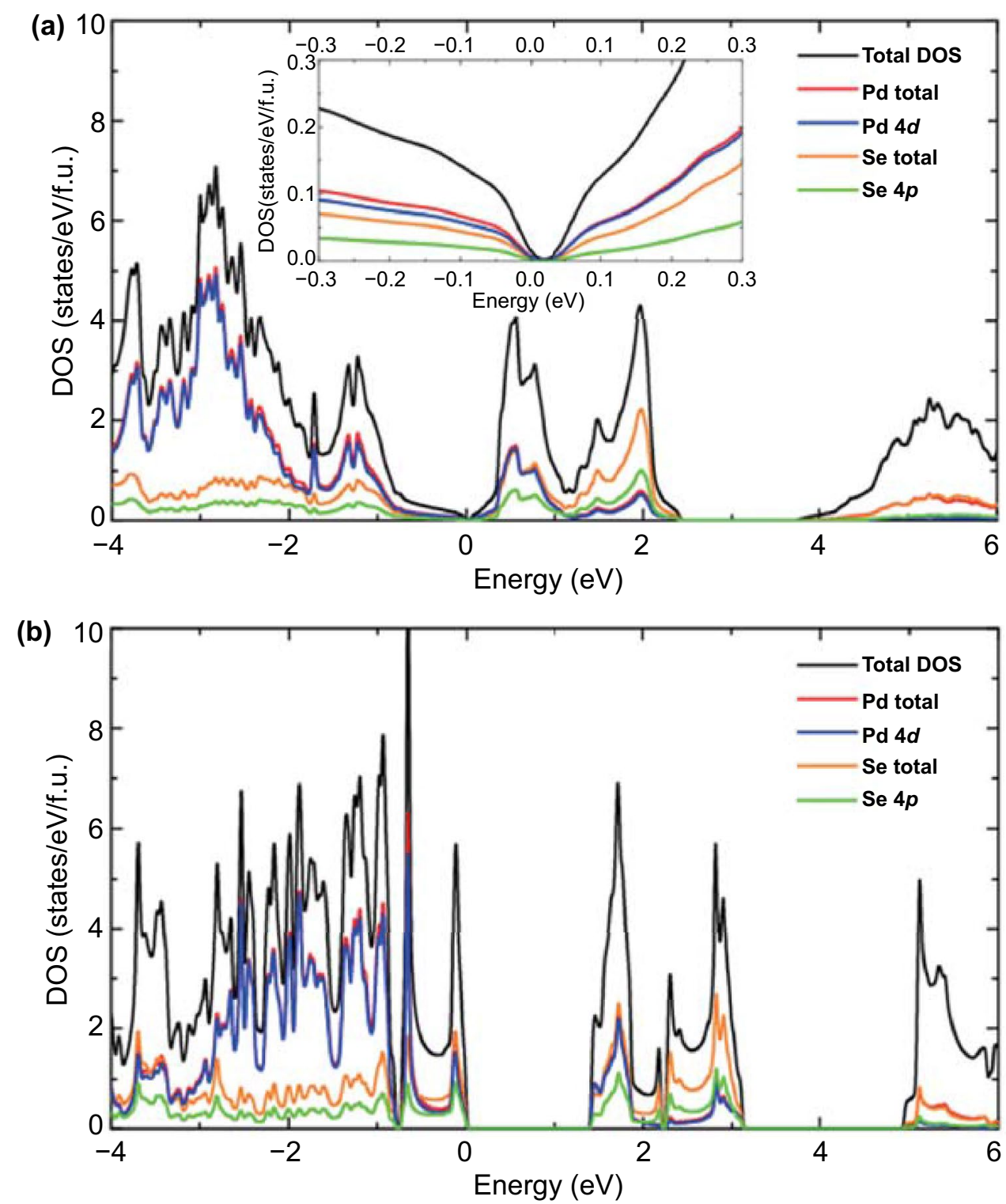

Fig. 3 Calculated density of states of a bulk $\mathrm{PdSe}_{2}$ and $\mathbf{b}$ monolayer $\mathrm{PdSe}_{2}$. "DOS" denotes the density of states. Reproduced with permission from Ref. [27]. Copyright 2015, AIP Publishing LLC

value. This uncertainty of the bandgap may be because $\mathrm{PdSe}_{2}$ has a high number of defects and in-plane anisotropic absorption properties.

In each layer, covalent bonding results in a distinct hybridization between the $\mathrm{Pd} 4 \mathrm{~d}$ and $\mathrm{Se} 4 \mathrm{p}$ states. The projected DOSs show that the $\mathrm{Pd} 4 \mathrm{~d}$ and $\mathrm{Se} 4 \mathrm{p}$ states contribute the most to the VBM and CBM, and the more substantial contribution of Pd $4 \mathrm{~d}$ orbitals to the total DOSs increases at an energy below $-1 \mathrm{eV}$ [27].

\subsection{Vibrational Phonon Modes}

Raman spectroscopy, which is a critical technique for $2 \mathrm{D}$ material characterization, was utilized to investigate the $\mathrm{PdSe}_{2}$ structure. In the Raman spectra of $\mathrm{PdSe}_{2}$, the peak position and intensity are shown to change anomalously with different numbers of $\mathrm{PdSe}_{2}$ layers, resulting from the electronic hybridization and strong interlayer coupling in the $\mathrm{PdSe}_{2}$ crystal [15]. 
To provide a better understanding, Fig. 4a shows the Raman spectra of $\mathrm{PdSe}_{2}$ samples from monolayer to bulk, which demonstrates the evolution of the $\mathrm{PdSe}_{2}$ vibrational modes. There are four obvious peaks in the high-frequency (HF) Raman spectra region (100-300 $\mathrm{cm}^{-1}$ ), including six atomic vibrational modes [15]. The six peaks are at 144.3, $146.9,206.7,222.7,257.8$, and $268.6 \mathrm{~cm}^{-1}$, and the corresponding $\mathrm{A}_{\mathrm{g}}{ }^{1}, \mathrm{~B}_{1 \mathrm{~g}}{ }^{1}, \mathrm{~A}_{\mathrm{g}}{ }^{2}, \mathrm{~B}_{1 \mathrm{~g}}{ }^{2}, \mathrm{~A}_{\mathrm{g}}{ }^{3}$, and $\mathrm{B}_{1 \mathrm{~g}}{ }^{3}$ phonon modes of $\mathrm{PdSe}_{2}$ are marked with dotted lines in Fig. 4a. As the number of $\mathrm{PdSe} \mathrm{e}_{2}$ layers increases, the major peaks show a red shift, with the $\mathrm{B}_{1 \mathrm{~g}}{ }^{1}$ peak changing the most and the $\mathrm{A}_{\mathrm{g}}{ }^{3}$ peak changing the least. The main reasons for this are the in-plane lattice constant variations and the strong interlayer coupling of $\mathrm{PdSe}_{2}$, which causes abnormal shifts and a broad bandgap [15].

Figure $4 \mathrm{~b}$ shows six atomic vibrational models, where the purple arrows represent the relative movements between the Pd and Se atoms. Among all the vibrational modes of $\mathrm{PdSe}_{2}$, the vibrations of $\mathrm{Se}-\mathrm{Se}$ atoms are predominant. Indeed, the $\mathrm{Se}-\mathrm{Se}$ bond presents a much stronger vibration intensity than that of the $\mathrm{Pd}-\mathrm{Se}$ bond [39]. Moreover, there are three peaks in the low-wavenumber region (approximately at 101, 121, and $130 \mathrm{~cm}^{-1}$ ) owing to variations in the symmetry. As the number of $\mathrm{PdSe}_{2}$ layers decreases, the space group transforms from Pbca to $P c a 2_{1}$, leading to the emergence of the $\mathrm{B}_{1 \mathrm{~g}}{ }^{3}$ mode and new peaks $\left(268.6 \mathrm{~cm}^{-1}\right)$ in few-layer $\mathrm{PdSe}_{2}$.

Low-frequency (LF) Raman spectroscopy $\left(<100 \mathrm{~cm}^{-1}\right)$ was used to study the layer characteristics of $\mathrm{PdSe}_{2}$ further. As the two primary LF features, the breathing and shear modes pertain to the interlayer vibrational modes, and they depend on the relative motion perpendicular and parallel to the atomic layers, respectively. The breathing modes $\left(\mathrm{BM}_{1}, \mathrm{BM}_{2}\right.$, and $\mathrm{BM}_{3}$ ) and shear modes (SM) are marked in Fig. 4a. For PdSe ${ }_{2}$, the intralayer covalent bonds along with the vibrational directions of adjacent atomic layers determine the intensities of the LF vibrational modes. Moreover, the interlayer vibrational modes display high intensities in few-layered $\mathrm{PdSe}_{2}$ flakes, even overtop the intralayer modes (HF features), which reflects the strong interlayer coupling of $\mathrm{PdSe}_{2}$. With the increase in the layer number of $\mathrm{PdSe}_{2}$, the LF Raman spectra exhibited a distinct red shift for the branches of the breathing modes. Such a shift was more pronounced than that of Raman peaks in the HF region. The full-width half-maximum (FWHM) of $\mathrm{BM}_{1}$ narrowed from $12 \mathrm{~cm}^{-1}$ (2 L) to $2.5 \mathrm{~cm}^{-1}$ $(7 \mathrm{~L})$ owing to the reduced phonon scattering rate in thicker

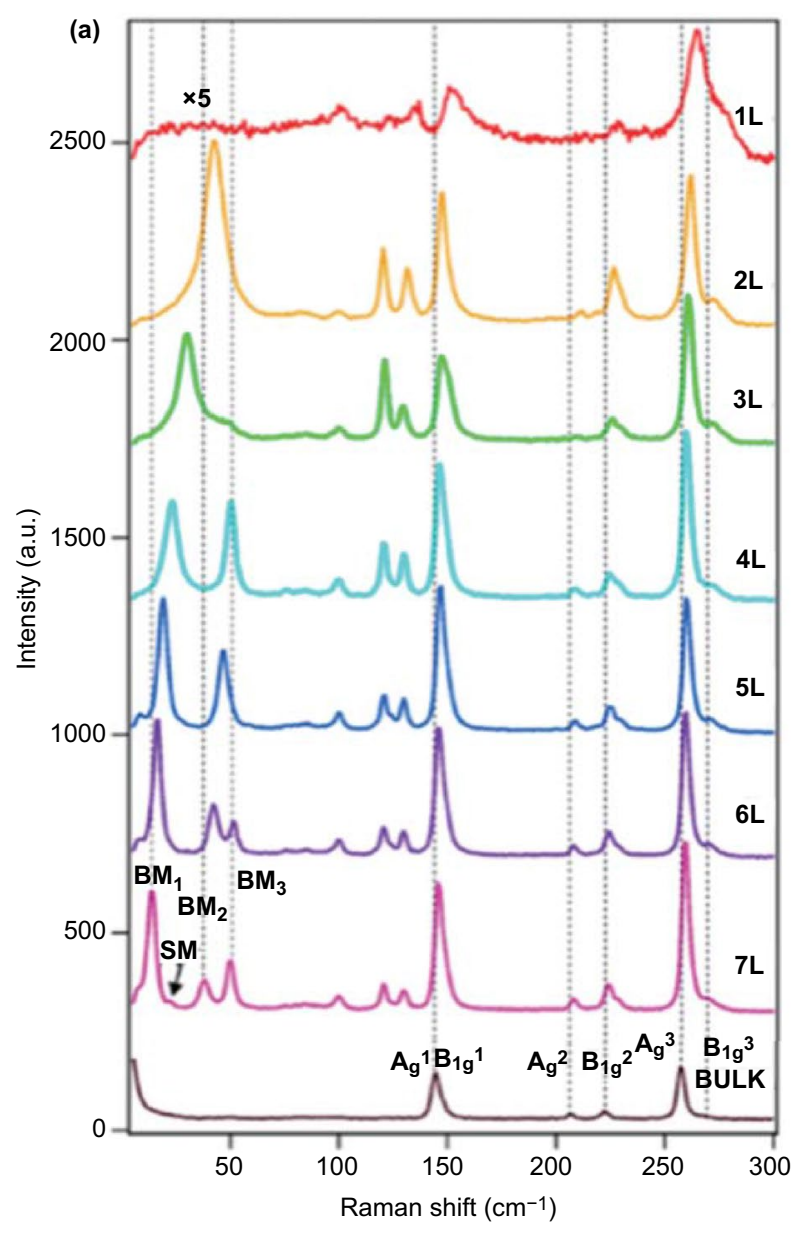

(b)

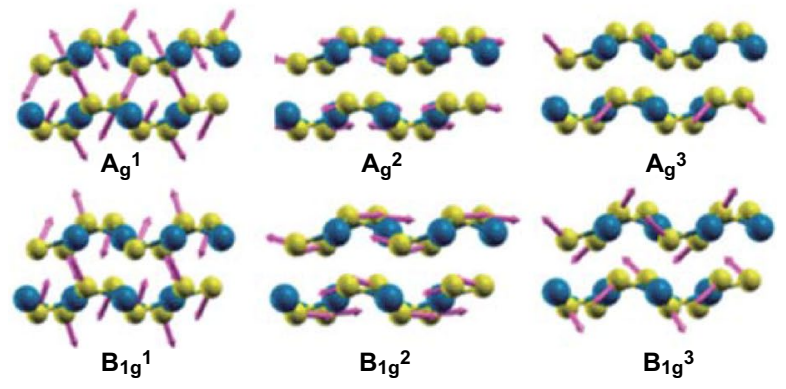

Fig. 4 Vibrational properties of $\mathrm{PdSe}_{2}$. a Raman spectra of $\mathrm{PdSe}_{2}$ flakes of different layer number from monolayer to bulk. b Six major vibrational modes of $\mathrm{PdSe}_{2}$, which are labeled as A1 g, B1 $1 \mathrm{~g}, \mathrm{~A} 2 \mathrm{~g}$, B2 $1 \mathrm{~g}, \mathrm{~A} 3 \mathrm{~g}$, and B3 $1 \mathrm{~g}$. Reprinted with permission from Ref. [39]. Copyright 2020, American Chemistry Society

$\mathrm{PdSe}_{2}$ flakes [18]. Thus, the number of $\mathrm{PdSe}_{2}$ layers can be precisely determined via Raman spectroscopy.

As mentioned above, $\mathrm{PdSe}_{2}$ presents relatively low symmetry owing to its puckered pentagonal structure, which exists in a few other TMDCs except $\mathrm{PdS}_{2}$. Thus, $\mathrm{PdSe}_{2}$ exhibits a unique anisotropy property, and the Raman 
scattering features of $\mathrm{PdSe}_{2}$ have been recently conducted to study the vibrational anisotropy [40].

\subsection{Polarization Properties}

Compared with 2D TMDCs, $\mathrm{PdSe}_{2}$ possesses unique optoelectronic polarization properties because of anisotropy [16, 40], which is a great advantage for detecting polarized light. The $\mathrm{PdSe}_{2}$ has an appropriate bandgap $(1.1 \mathrm{eV})$ and excellent optical absorption at the near-infrared range [40].

To date, $\mathrm{PdSe}_{2}$ remains the only choice for polarization investigation among the noble metal dichalcogenides. Indeed, the pentagonal $\mathrm{PdS}_{2}$ may possess the photoelectric properties analogous to the $\mathrm{PdSe}_{2}$. But $2 \mathrm{D} \mathrm{PdS}$ investigation remains the theoretical calculation [13] and has yet been successfully prepared in experiments. This is probably because of the thermodynamic instability of marcasite $\mathrm{PdS}_{2}$ in the air [14].
Therefore, the application of $\mathrm{PdSe}_{2}$ exhibits high promise in the applications of optoelectronics and electronics.

Polarization-resolved Raman measurements and theoretical calculations were employed to systematically investigate the anisotropic optical properties [39]. Figure 5a, b shows the Raman intensity simulations of the $\mathrm{A}_{\mathrm{g}}$ and $\mathrm{B}_{1 \mathrm{~g}}$ modes versus the polarization angle in $3 \mathrm{~L} \mathrm{PdSe} e_{2}$ under parallel polarization configuration. The $\mathrm{A}_{\mathrm{g}}$ modes reveal a period of $180^{\circ}$, and the $B_{1 \mathrm{~g}}$ modes reveal a period of $90^{\circ}$ in the parallel configuration.

Figure $5 \mathrm{c}, \mathrm{d}$ presents the Raman intensity of both modes under parallel polarization configuration. Indeed, the $A_{g}$ and $B_{1 \mathrm{~g}}$ modes both reveal a period of $90^{\circ}$ under the cross configuration. The LF Raman peaks possess $A_{g}$ or $B_{1 g}$ symmetry because the LF modes follow the group theory, similar to the HF modes, and the breathing modes and shear modes possess $\mathrm{A}_{\mathrm{g}}$ and $\mathrm{B}_{1 \mathrm{~g}}$ symmetry, respectively.
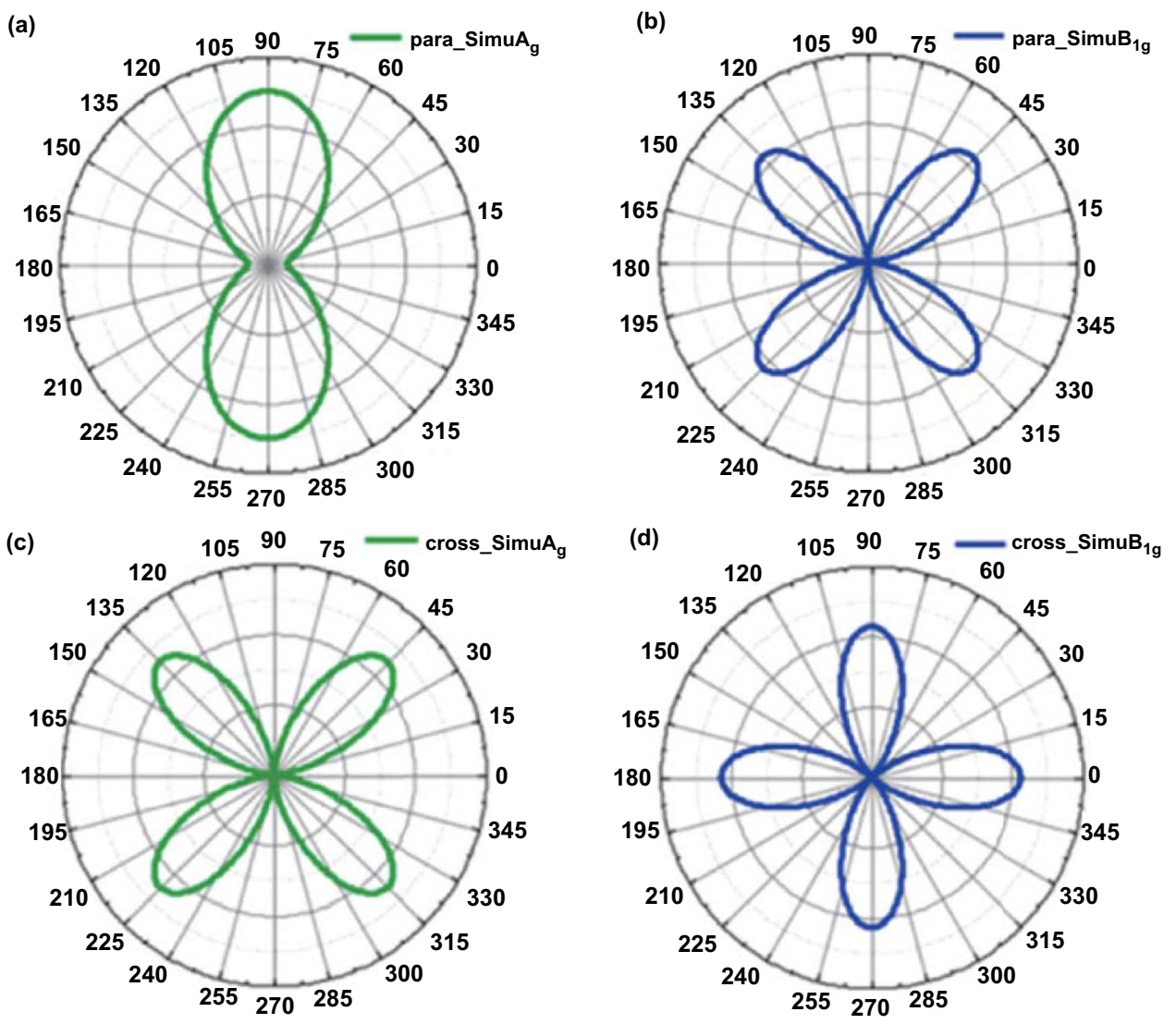

Fig. 5 Polarization Raman intensities of $\mathrm{PdSe}_{2}$. The Raman intensity of $\mathrm{A}_{\mathrm{g}}$ mode (a) and $\mathrm{B}_{1 \mathrm{~g}}$ mode (b) under the parallel configuration with the simulation of the anisotropic modes. Raman intensity of $\mathrm{A}_{\mathrm{g}}$ mode $(\mathbf{c})$ and $\mathrm{B}_{1 \mathrm{~g}}$ mode (d) under cross configuration of polarization Raman test. The layer number of $\mathrm{PdSe}_{2}$ is 3 for polarization Raman test. Reprinted with permission from Ref. [39]. Copyright 2020, American Chemistry Society 


\subsection{Optical Absorption Properties}

The anisotropic features of $\mathrm{PdSe}_{2}$ can be verified based on its optical absorption. Figure 6a shows the optical absorbance of 1-3 $\mathrm{L} \mathrm{PdSe}_{2}$ flakes at measurement angles of $0^{\circ}$ and $90^{\circ}$, where an interesting orthogonal crossover is observed at around $470 \mathrm{~nm}$ [39]. Owing to the decrease in the bandgap, the increase in the number of $\mathrm{PdSe}_{2}$ layers leads to a slight red shift of the intersection point after $600 \mathrm{~nm}$.

Figure $6 \mathrm{~b}$ shows the variation in $\mathrm{PdSe}_{2}$ absorption with the polarization angle for a systematic investigation of the anisotropic characteristics. Almost all the absorption spectra of $\mathrm{PdSe}_{2}$ intersect at $472 \mathrm{~nm}$ when the polarization angle varies from $-90^{\circ}$ to $90^{\circ}$.

\subsection{Photoelectronic Properties}

Based on the optical absorption of $\mathrm{PdSe}_{2}$, the photoresponse of $2 \mathrm{D} \mathrm{PdSe}_{2}$ was investigated. The spatially resolved photocurrent mapping was collected for the fewlayer $\mathrm{PdSe}_{2}$ devices [41]. Figure 4g shows a stable photocurrent of the device under 1060-nm illumination at two metal-PdSe ${ }_{2}$ junctions without any applied voltage.

To further study the photocurrent generation mechanisms, gate-dependent scanning photocurrent measurements were taken (Fig. 7a, b). Besides, the photocurrent could be tuned from positive to negative when regulating the drain-source voltage from 150 to $-150 \mathrm{mV}$ (Fig. 7c). The photocurrent mapping could be applied in the image sensing.

A strong photocurrent resonance peak emerges at $1060 \mathrm{~nm}$, which may be due to an indirect optical transition. Due to the potential barriers created by the Fermi level alignment, a built-in electric field separates the photogenerated electron-hole pairs in the $\mathrm{PdSe}_{2}$ device [41].

\subsection{Thermoelectric Properties}

Over the past decade, thermoelectric devices have attracted much attention because they can directly convert thermal energy into electrical energy. Because the bond saturation significantly enhances the thermal energy transport in $2 \mathrm{D}$ pentagonal materials, a unique feature is that $\mathrm{PdSe}_{2}$ possesses good thermoelectric properties. In particular, monolayer $\mathrm{PdSe}_{2}$ can be applied as a promising highperformance thermoelectric material in the future owing to its high Seebeck coefficient $\left(>200 \mu \mathrm{V} \mathrm{K}^{-1}\right.$ ) [27]. For few-layer $\mathrm{PdSe}_{2}$, the energies of $\mathrm{CB}$ and VB were found to be convergent during a systematic investigation of its lattice structure and electronic properties, which indicates the significant thermoelectric properties of $\mathrm{PdSe}_{2}$ [42].

Figure 8a shows the electron transport coefficient of $\mathrm{PdSe}_{2}$ based on the constant relaxation time approximations of the Boltzmann theory [39]. Clearly, when the
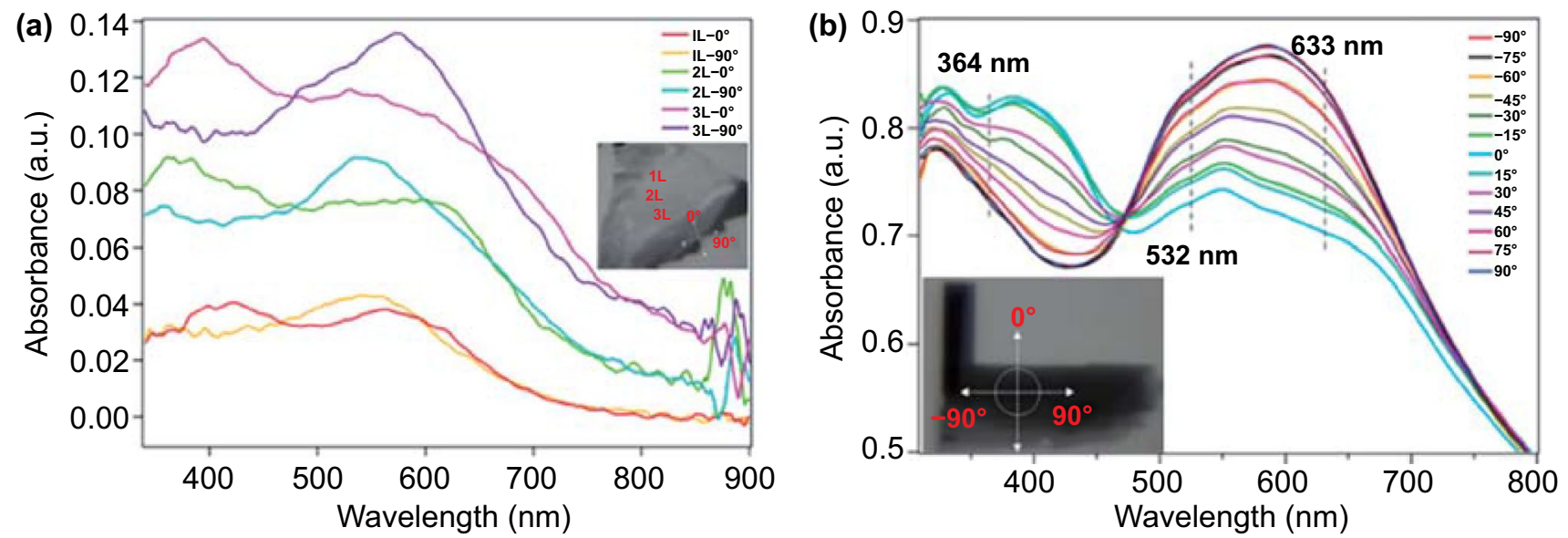

Fig. 6 Polarized optical absorption of $\mathrm{PdSe}_{2}$. a Absorbance of 1-3 $\mathrm{L} \mathrm{PdSe}_{2}$ along the $x$-axis $\left(90^{\circ}\right)$ and y-axis $\left(0^{\circ}\right)$. Inset: Optical micrograph of the $\mathrm{PdSe}_{2}$ flakes of different thicknesses. b Polarization-resolved absorption spectra of bulk $\mathrm{PdSe}_{2}$ within $300-800 \mathrm{~nm}$ spectra, with the measured angle from -90 to $90^{\circ}$ in increments of $15^{\circ}$. Inset: Optical micrograph of the $\mathrm{PdSe}_{2}$ sample. Reprinted with permission from Ref. [39]. Copyright 2020, American Chemistry Society 

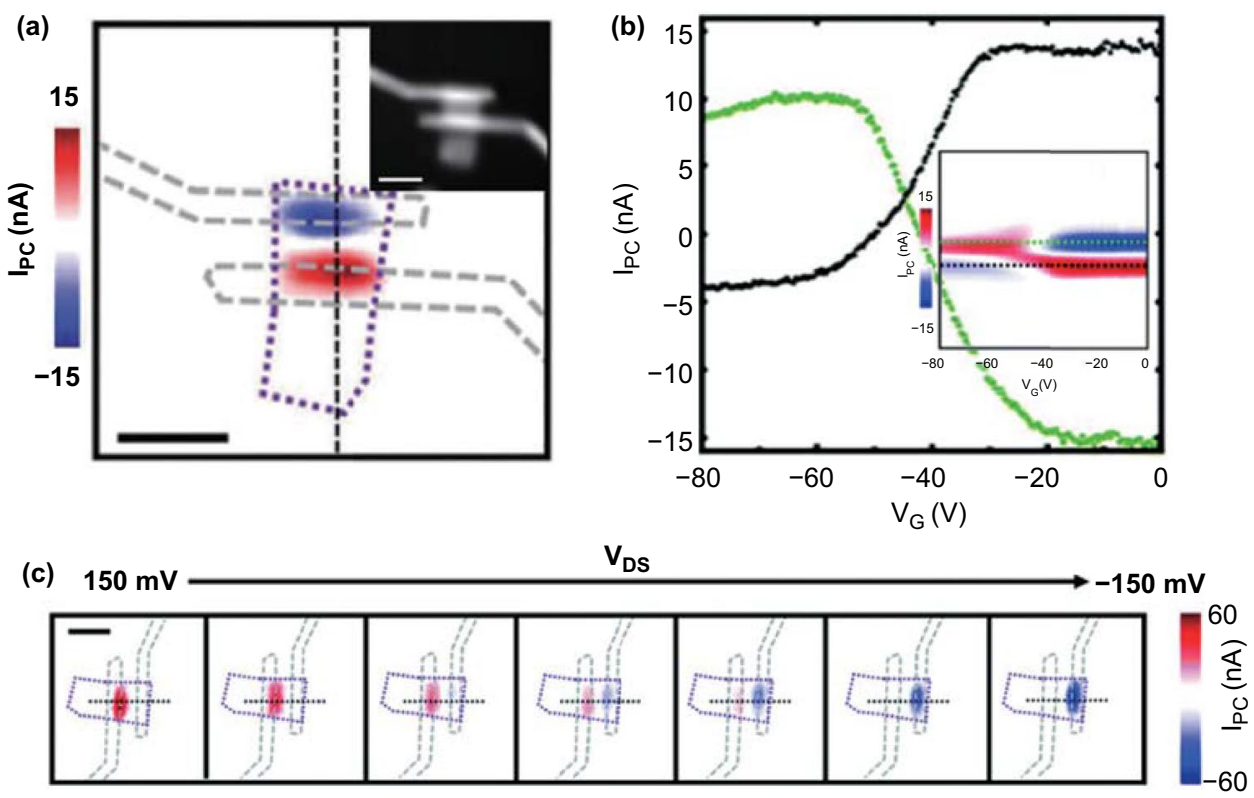

Fig. 7 Photoelectric current mapping of $\mathrm{PdSe}_{2}$. a Scanning photocurrent images of the $\mathrm{PdSe}_{2}$ device under 1060-nm illumination with $\mathrm{V}_{\mathrm{G}}=\mathrm{V}_{\mathrm{DS}}=0 \mathrm{~V}$, where the scale bar represents $5 \mu \mathrm{m}$. Inset: Reflection image of corresponding device with scale bar of $5 \mu \mathrm{m}$. b Photocurrents along the green and black dashed lines. Inset: Photocurrent signals as a function of gate voltage along the black dashed line in a. c Scanning photocurrent images of the $\mathrm{PdSe}_{2}$ device in a with $\mathrm{V}_{\mathrm{DS}}$ from -150 to $150 \mathrm{mV}$, where the scale bar represents $5 \mu \mathrm{m}$. Reprinted with permission from Ref. [41]. Copyright 2019, Royal Society of Chemistry

doped carrier concentration increased, the conductivity $(\sigma)$ increased, while the Seebeck coefficient decreased. For monolayer PdSe $e_{2}$, the Seebeck coefficient can reach $660 \mu \mathrm{V} \mathrm{K}^{-1}$, which is comparable to that of some reported 2D materials [43]. The $\mathrm{S}$ for $p$-type doping is more asymmetric than that for $n$-type doping, and this provides the possibility for the design of transverse thermoelectric devices. Figure $8 \mathrm{a}$ proves that the power factor $(\mathrm{PF}) \mathrm{S}^{2} \sigma$ possesses distinct anisotropy, and this results from the large anisotropy of $\sigma$ and $S$.

Figure $8 \mathrm{~b}$ shows the calculation of the lattice thermal conductivity $\kappa_{1}$ through the phonon Boltzmann transport equation and DFT. The lattice thermal conductivity of $\mathrm{PdSe}_{2}$ is much lower than that of monolayer $\mathrm{MoS}_{2}$ and $\mathrm{GX}_{2}$ [44], and it exhibits a large directional anisotropy. Figure $8 \mathrm{c}$ displays the relationship between the dimensionless figure of merit (ZT) value of the doped monolayer $\mathrm{PdSe}_{2}$ and the carrier concentration at room temperature.

The ZT value of monolayer $\mathrm{PdSe}_{2}$ is small and almost isotropic, while that for $p$-type doping is large and strongly anisotropic. Therefore, the high $\mathrm{S}$, low $\sigma$, and high $\mathrm{ZT}$ values of monolayer $\mathrm{PdSe}_{2}$ at room temperature make $\mathrm{PdSe}_{2}$ suitable for thermoelectric devices.

\subsection{Phase Transformation Properties}

Two-dimensional materials, especially TMDCs, can possess various properties via change in their phases, namely in terms of bonding and configurations, which can be exploited in other fields. For $\mathrm{PdSe}_{2}$, the interlayer interaction is relatively more reliable than the intralayer connection through covalent bonds, which facilitates the transition to other phases under different external parameters. The unique puckered pentagonal structure of $\mathrm{PdSe}_{2}$ possesses imperfect rotational symmetry, resulting in high defect sensitivity, particularly Se vacancies $\left(\mathrm{V}_{\mathrm{Se}}\right)$, which facilitates the occurrence of different phase transitions [45].

$\mathrm{PdSe}_{2}$ structure could transform into a $\mathrm{Pd}_{2} \mathrm{Se}_{3}$ structure (Fig. 9a) through $\mathrm{V}_{\mathrm{Se}}$ [46]. From the STEM images, it was found that the preferred monolayer phase form exfoliated from bulk $\mathrm{PdSe}_{2}$ is not a $\mathrm{PdSe}_{2}$ structure. Through analysis of the quantitative STEM image intensity and DFT calculations, a new stable monolayer phase was determined to be $\mathrm{Pd}_{2} \mathrm{Se}_{3}$, which corresponds to the result from the experimental ADF-STEM image (Fig. 9b) [47]. 

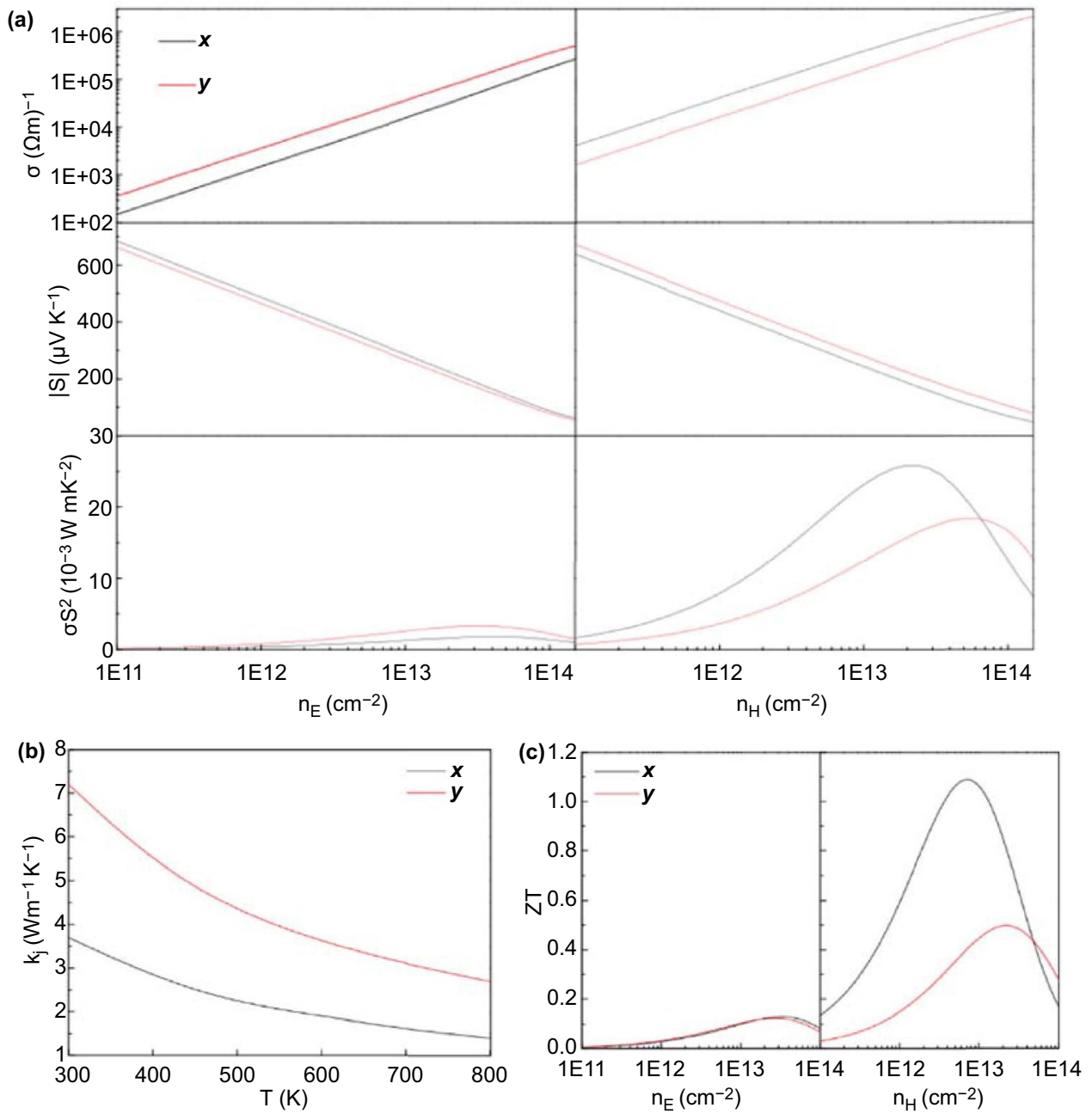

Fig. 8 Thermoelectric properties of $\mathrm{PdSe}_{2}$. a Thermoelectric transport coefficients $\sigma, \mathrm{S}$, and $\mathrm{S}^{2} \sigma$ versus carrier concentration for PdSe $e_{2}$ with $n$-type (left) and $p$-type (right) doping at room temperature. b Lattice thermal conductivity of monolayer PdSe $\mathrm{P}_{2}$ as a function of temperature. c Thermoelectric characteristics (ZT) of monolayer $\mathrm{PdSe}_{2}$ with $n$-type (left) and $p$-type doping (right) at room temperature. Adapted under the terms of the CC-BY Creative Commons Attribution 4.0 license (https://creativecommons.org/licenses/by/4.0/) from Ref. [138]. Copyright 2018, The Authors, published by Springer Nature

The reconstruction of $\mathrm{Pd}_{2} \mathrm{Se}_{3}$ is due to the interlayer fusion mechanism, which results from the $\mathrm{V}_{\mathrm{Se}}$ produced by electron radiation (Fig. 9c). According to the research results, the new $\mathrm{Pd}_{2} \mathrm{Se}_{3}$ phase exhibits physical stability and high cohesive energy, implying robust chemical bonding. Moreover, the $\mathrm{Pd}_{2} \mathrm{Se}_{3}$ monolayer is an excellent thermoelectric material with good electronic and optical properties [48].

Figure $9 \mathrm{~d}$ shows the typical $\mathrm{V}_{\mathrm{Se}}$ migration process in $\mathrm{PdSe}_{2}$ in four possible configurations labeled I, II, III, and IV. The red circle indicates the position of the $\mathrm{V}_{\mathrm{Se}}$, which diffuses in the direction of the green arrow. The theoretically calculated energy barriers were presented for the corresponding $\mathrm{V}_{\mathrm{Se}}$ diffusion (Fig. 9e). For configurations I to II and III to IV, the energy barrier of interlayer and intralayer $\mathrm{V}_{\mathrm{Se}}$ diffusion is 1.59 and $1 \mathrm{eV}$, respectively. These barriers are lower than the corresponding energy barriers in $\mathrm{MoS}_{2}$. These $\mathrm{V}_{\mathrm{Se}}$ migrations are facilitated by the stronger interlayer interaction and weaker intralayer bond strength of $\mathrm{PdSe}_{2}$. For configurations II to III, the energy barrier for intralayer $\mathrm{V}_{\mathrm{Se}}$ diffusion is $0.03 \mathrm{eV}$ owing to the Se-Se bonding [45].

Environmental energy input elevates the energy of $\mathrm{PdSe}_{2}$ and provides the activation energy for the formation 
(a)

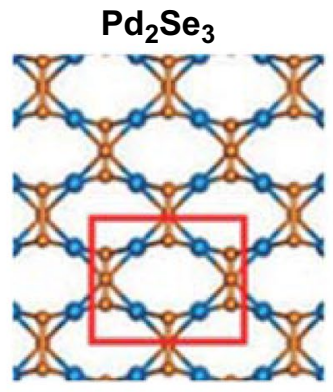

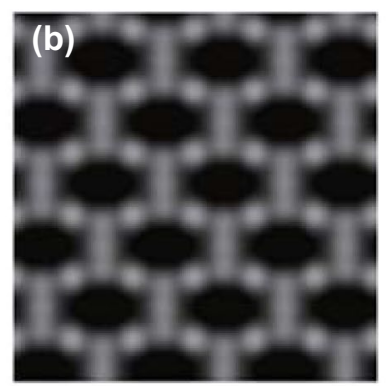

(d)
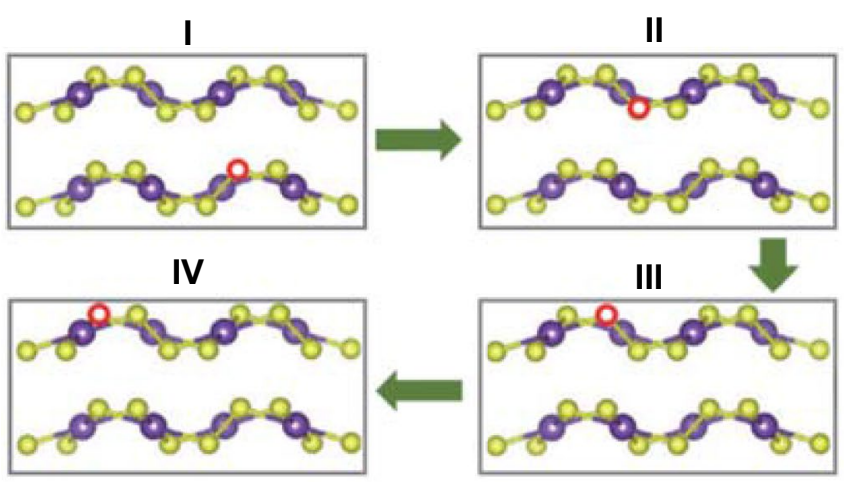

(c)
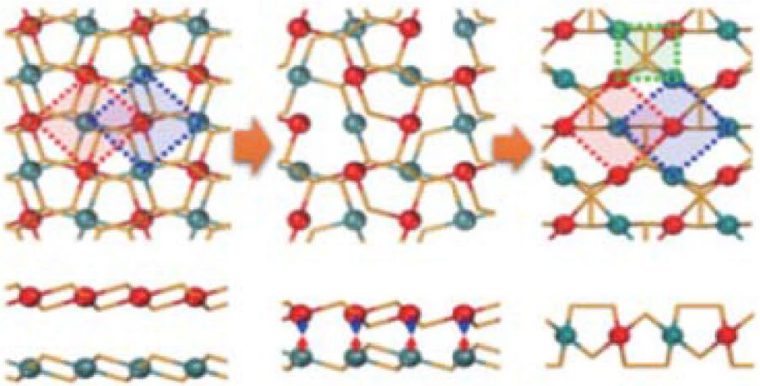

(e)

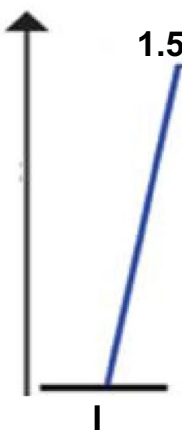

$1.59 \mathrm{eV}$

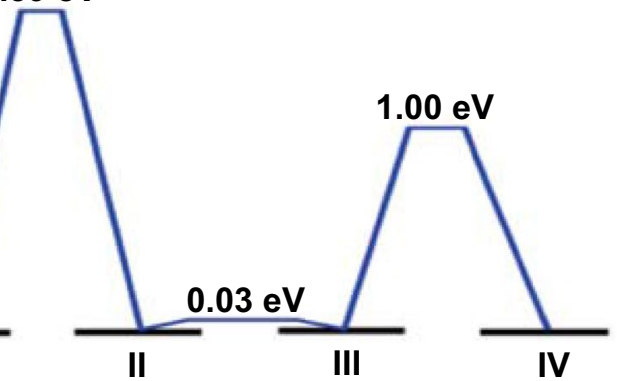

(f)

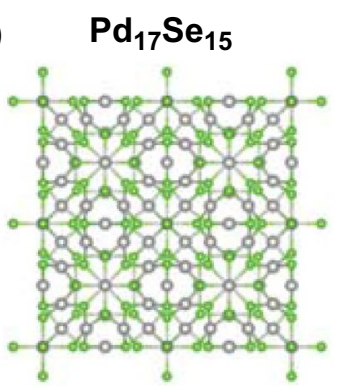

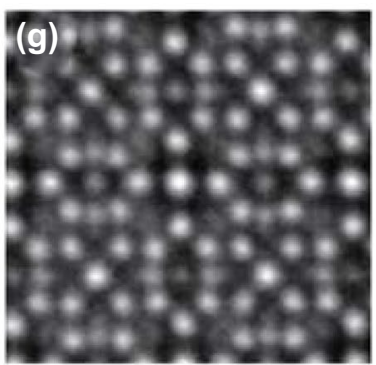

(h)

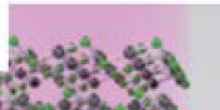

$\mathrm{Ar}^{+}$plasma

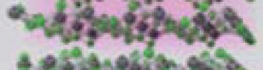

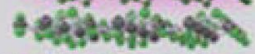

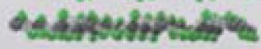

+ \$)

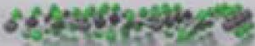

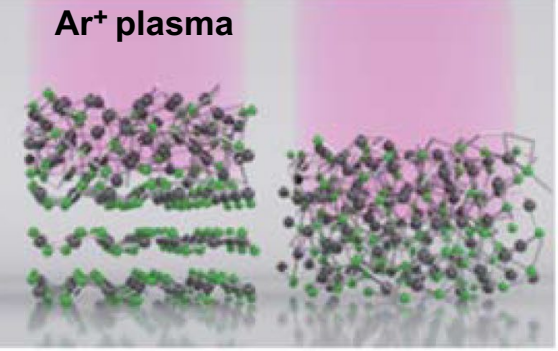

Fig. 9 Atomic structure of different palladium selenide compounds. a Lattice structures and b corresponding simulated ADF-STEM image of monolayer $\mathrm{Pd}_{2} \mathrm{Se}_{3}$. Reprinted with permission from Ref. [47]. Copyright 2019, American Chemistry Society. c Schematic of reconstruction mechanism from bilayer $\mathrm{PdSe}_{2}$ to monolayer $\mathrm{Pd}_{2} \mathrm{Se}_{3}$, where the Se atoms are not presented. Reprinted with permission from Ref. [46]. Copyright 2017, American Physical Society. d Migration of $\mathrm{V}_{\mathrm{Se}}$ configuration marked with the red circle in layered PdSe $e_{2}$. e Energy barriers of $\mathrm{V}_{\mathrm{Se}}$ diffusions calculated between different configurations. Reprinted with permission from Ref. [45]. Copyright 2017, American Physical Society. f Lattice structures and $\mathbf{g}$ corresponding ADF-STEM image of $\mathrm{Pd}_{17} \mathrm{Se}_{15}$, where green and gray spheres represent Se atoms and Pd atoms, respectively. h Process diagram of $\mathrm{Pd}_{17} \mathrm{Se}_{15}$ formation from PdSe 2 layer-by-layer through Ar plasma treatment. Reprinted with permission from Ref. [52]. Copyright 2019, American Chemistry Society

of other Pd-Se compounds, viz. the phase transformation occurs. For example, the thermal annealing, plasma, and laser treatment have resulted in the phase transition of $\mathrm{PdSe}_{2}$. The typical external conditions are listed in Table 2 for the phase transition of $\mathrm{PdSe}_{2}$.

First, $\mathrm{PdSe}_{2}$ can be transformed to $\mathrm{PdSe}_{2-x}$ with vacuum annealing. According to the traditional bulk Pd-Se phase diagram [49], the Se loss induces the change in the $\mathrm{Pd} / \mathrm{Se}$ ratio. Hence, the phase transition occurs after 30-s pulse annealing at $400{ }^{\circ} \mathrm{C}$ and the $\mathrm{PdSe}_{2-x}(x=0-1)$ forms partially. Another 30 -s pulse annealing completed the phase transition into $\mathrm{Pd}_{2} \mathrm{Se}_{3}$. The long-time annealing at $400{ }^{\circ} \mathrm{C}$ or heating at high temperature $\left(>400^{\circ} \mathrm{C}\right)$ leads to excess Se loss and thinning of 2D materials and finally form pure Pd materials [49]. Indeed, Se loss occurs in other metal selenide upon thermal annealing. Second, high laser power can lead to Se 
Table 2 The phase transition of $\mathrm{PdSe}_{2}$ under different conditions

\begin{tabular}{|c|c|c|c|}
\hline Phase transition from starting phase & To final phase & Conditions & References \\
\hline Pristine $\mathrm{PdSe}_{2}(2-4 \mathrm{~L})$ & Defective $\mathrm{PdSe}_{2}$ (Se vacancy) & $400{ }^{\circ} \mathrm{C}$ annealing in vacuum for $10 \mathrm{~s}$ & [49] \\
\hline Defective $\mathrm{PdSe}_{2}$ & $50 \% \mathrm{PdSe}_{2}+50 \% \mathrm{PdSe}_{2-x}(x=0-1)$ & $400{ }^{\circ} \mathrm{C}$ in vacuum for $30 \mathrm{~s}$ & [49] \\
\hline $\operatorname{Partial~PdSe}_{2-x}(x=0-1)$ & $100 \% \mathrm{PdSe}_{2-x}$ & $400{ }^{\circ} \mathrm{C}$ in vacuum for $30 \mathrm{~s}$ & [49] \\
\hline $\operatorname{PdSe}_{2-x}(x=0-1)$ & $\mathrm{Pd}_{2} \mathrm{Se}_{3}$ (striated; $1 \mathrm{D}$ channels) & $400{ }^{\circ} \mathrm{C}$ heating in vacuum & [49] \\
\hline $\mathrm{Pd}_{2} \mathrm{Se}_{3}$ & PdSe & Vacuum annealing for Se loss & [49] \\
\hline PdSe & Pd nanoparticles & $\begin{array}{l}\text { Long vacuum annealing at } 400{ }^{\circ} \mathrm{C} \text { or } \\
\text { heating at high temperatures }\left(>400{ }^{\circ} \mathrm{C}\right)\end{array}$ & [49] \\
\hline $\mathrm{PdSe}_{2}$ & $\mathrm{Pd}_{17} \mathrm{Se}_{15}$ & Ar plasma treatment & {$[52]$} \\
\hline $\mathrm{PdSe}_{2}$ & $\operatorname{PdSe}_{2-x}(x=0-1)$ & Laser irradiation $(60 \mu \mathrm{W})$ & {$[50]$} \\
\hline $\mathrm{PdSe}_{2}$ & Pd nanoparticles & Laser irradiation $(600 \mu \mathrm{W})$ & {$[50]$} \\
\hline 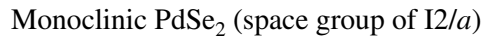 & Monoclinic $\mathrm{PdSe}_{2}(\mathrm{C} 2 / \mathrm{m}$ space group $)$ & High pressure (4.5 GPa) & {$[40]$} \\
\hline $\mathrm{PdSe}_{2}$ & Hexagonal $\mathrm{PdSe}_{2}(\mathrm{P}-3 \mathrm{~m} 1$ space group $)$ & High pressure (17.5 GPa) & {$[40]$} \\
\hline Orthorhombic $\mathrm{PdSe}_{2}$ & $\begin{array}{c}\text { Ferroelastic } \mathrm{PdSe}_{2} ; \text { Transition of layer } \\
\text { stacking from } c \text { to a-axis orientation }\end{array}$ & Uniaxial compressive stress ( $0.6 \mathrm{GPa})$ & {$[33]$} \\
\hline
\end{tabular}

loss and the formation of Pd nanoparticles [50]. Third, the high-pressure condition may induce the change of crystal structures [40] and layer stacking orientation [33].

Except for $\mathrm{Pd}_{2} \mathrm{Se}_{3}$, the $\mathrm{Pd}-\mathrm{Se}$ binary phases include $\mathrm{Pd}_{17} \mathrm{Se}_{15}, \mathrm{Pd}_{7} \mathrm{Se}_{4}$, and $\mathrm{Pd}_{4} \mathrm{Se}$. Through experiments, their metallic or superconducting characteristics have been displayed, and theoretical predictions have highlighted their topological quantum properties [51].

For instance, the $\mathrm{Pd}_{17} \mathrm{Se}_{15}$ phase has excellent stability with analogous chemical bonds to those of the $\mathrm{PdSe}_{2}$ phase [52]. Figure 9f, g shows the structure of the $\mathrm{Pd}_{17} \mathrm{Se}_{15}$ phase and the corresponding STEM images. The phase transition results from the $\mathrm{V}_{\mathrm{Se}}$ in the $\mathrm{PdSe}_{2}$ crystal are due to Arplasma treatment (Fig. 9h). Moreover, the Raman spectra and STEM images indicate that the exposure time under Ar plasma irradiation affects the defects and degree of the phase transition in the $\mathrm{PdSe}_{2}$ crystal.

We now come to the introduction of synthesis strategies and posttreatment approaches.

\section{Synthesis Methods for Obtaining $\mathrm{PdSe}_{2}$}

High-quality $\mathrm{PdSe}_{2}$ has been obtained via several reliable methods [17], which shows promise for exploration of its remarkable properties. In this section, we review the specific $\mathrm{PdSe}_{2}$ synthesis methods in terms of 3D bulk crystals and 2D thin films.

\subsection{Formation of 3D Bulk Crystals via Chemical Vapor Transport}

The chemical vapor transport (CVT) method has been developed for the synthesis of most 3D bulk materials; it is an efficient method employed for laboratory synthesis and mass production. A common CVT reaction involves three processes: sublimation, transport, and deposition, and follows Le Chatelier's principle in thermodynamics [53].

The typical chemical vapor transport method has shown success in the growth of bulk $\mathrm{PdSe}_{2}$ crystals [54]. Herein, a stoichiometric ratio of high-purity $\mathrm{Pd}$ and Se powder was mixed as the source and placed into an ampoule reactor with mineralizers as the transporting agent (Fig. 10). The sealed reactor was then heated under a preset temperature gradient, where Temperature 1 is the temperature for the sublimation of $\mathrm{Pd}$ and $\mathrm{Se}$ and Temperature 2 is the temperature for $\mathrm{PdSe}_{2}$ deposition [54]. Generally, Temperature 1 is greater than Temperature 2 because the process of $\mathrm{PdSe}_{2}$ crystal formation is endothermic [53].

For example, $\mathrm{Pd}$ and Se powders (mixed in an atomic ratio of 1:6) were filled in a sealed evacuated quartz ampule, which was slowly heated to $850{ }^{\circ} \mathrm{C}$ and maintained for $50 \mathrm{~h}$. After the synthesis was completed, the quartz ampule was gradually cooled to $450{ }^{\circ} \mathrm{C}$ at a rate of $3{ }^{\circ} \mathrm{C} \mathrm{h}^{-1}$ and finally naturally cooled to room temperature [53]. Eventually, shiny single $\mathrm{PdSe}_{2}$ crystals were obtained on millimeter-grade paper. 
Temperature 1

Temperature 2

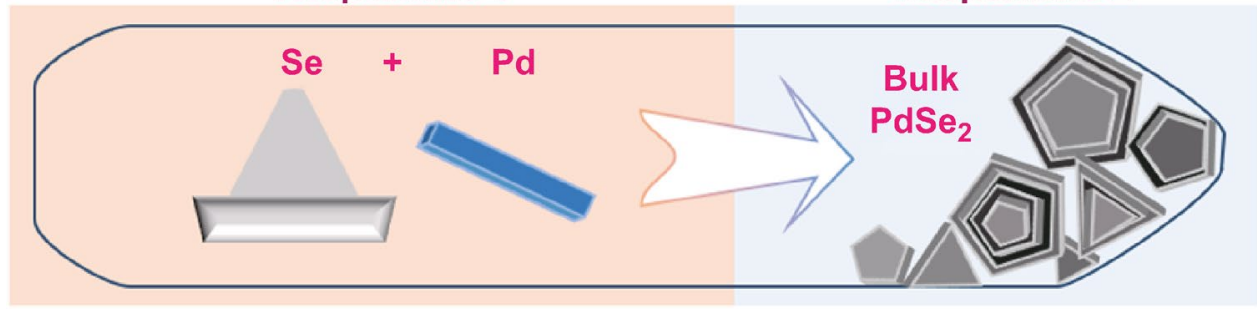

Fig. 10 Scheme of the chemical vapor transport method for the bulk $\mathrm{PdSe}_{2}$ formation. The selenium power and Pd metal are sublimated in the left heating zone (Temperature 1) and cooling in the cold zone (Temperature 2) as bulk. The drawing was inspired by the literature [53]. The scheme was originally drawn by the authors in this review

\subsection{Developing 2D Thin Tilm via Exfoliation}

As devices with smaller sizes and higher performance are desired in the development of electronics, the growth of high-quality ultrathin 2D materials has become increasingly crucial. Thus, mechanical exfoliation and chemical vapor deposition (CVD) techniques are widely employed to produce layered $\mathrm{PdSe}_{2}$ thin films.

After the synthesis of bulk $\mathrm{PdSe}_{2}$ crystals, atomic $\mathrm{PdSe}_{2}$ thin flakes could be easily obtained using the mechanical exfoliation method [15]. $\mathrm{PdSe}_{2}$ flakes with different layers were transformed onto the $\mathrm{Si} / \mathrm{SiO}_{2}$ substrate (Fig. 11). The exfoliated $\mathrm{PdSe}_{2}$ samples were then applied to different electronic devices.

The exfoliated $\mathrm{PdSe}_{2}$ flakes have high crystallinity (Fig. 11) and intrinsic properties, which are beneficial for fabrication of individual devices [39]. The mechanical exfoliation method enables facile fabrication of the vdWHs [54]. However, the lack of large-area uniformity and layer-number controllability limits the applicability of the mechanical exfoliation method; moreover, the method is difficult to use for industrial production.

The typical features are compared in Table 3 for the synthesis approaches of $3 \mathrm{D}$ bulk, nanosheets, and 2D films of $\mathrm{PdSe}_{2}$.

Recently, the Au-assisted exfoliation method has shown success in the separation of monolayer 2D materials over a centimeter size [55]. In brief, the Au film is first deposited onto a target substrate [56]. Then, the tape with exfoliated 2D material is stuck onto the Au surface. Upon the pressing over the sample, the strong interaction forms between $\mathrm{Au}$ and 2D material. Eventually, monolayer or few-layer 2D materials remain over the Au surface after peeling off the tape. Here, the interlayer interaction in TMDCs can be overcome by the interaction between Au film and 2D materials [57]. The strong van der Waals interaction between $\mathrm{Au}$ and the uppermost two-dimensional layered transitionmetal chalcogenide promotes the exfoliation of the single layer, which leaves large-area single-layer domain on the $\mathrm{Au}$ surface. For example, Au-assisted exfoliation has produced large $\mathrm{MoS}_{2}$ domains, i.e., 40 times greater than that produced by the tape-assisted exfoliation [57].

The Au-assisted exfoliation has become a universal approach for obtaining millimeter-sized 2D materials including $\mathrm{PtSe}_{2}, \mathrm{PtTe}_{2}$, and $\mathrm{PdTe}_{2}$ [58]. It may apply to the exfoliation of $\mathrm{PdSe}_{2}$ over a large size soon, which may accelerate the fabrication of electronic device arrays due to the large effective film area. The 2D materials over Au film by $\mathrm{Au}$ assisted exfoliation can be applied in electrochemistry and photocatalyst [55].

Most 2D materials with large-area uniformity and high crystallinity can be synthesized via the CVD method or thermal selenization/sulfurization treatment [59]. Several approaches have been used to grow homogeneous $\mathrm{PdSe}_{2}$ thin films, with satisfactory results being obtained. We now discuss thermal deposition approaches for synthesizing $\mathrm{PdSe}_{2}$ films.

\subsection{Chemical Vapor Deposition from the $\mathrm{PdCl}_{2}$ and Se Reaction}

A chemical vapor deposition strategy was developed by employing Pd-containing precursors and Se powders for synthesizing the $\mathrm{PdSe}_{2}$ films. Here, $\mathrm{PdCl}_{2}$ powder was selected as precursors [60]. A schematic of the CVD process with a three-zone tube furnace is shown in Fig. 12a. 

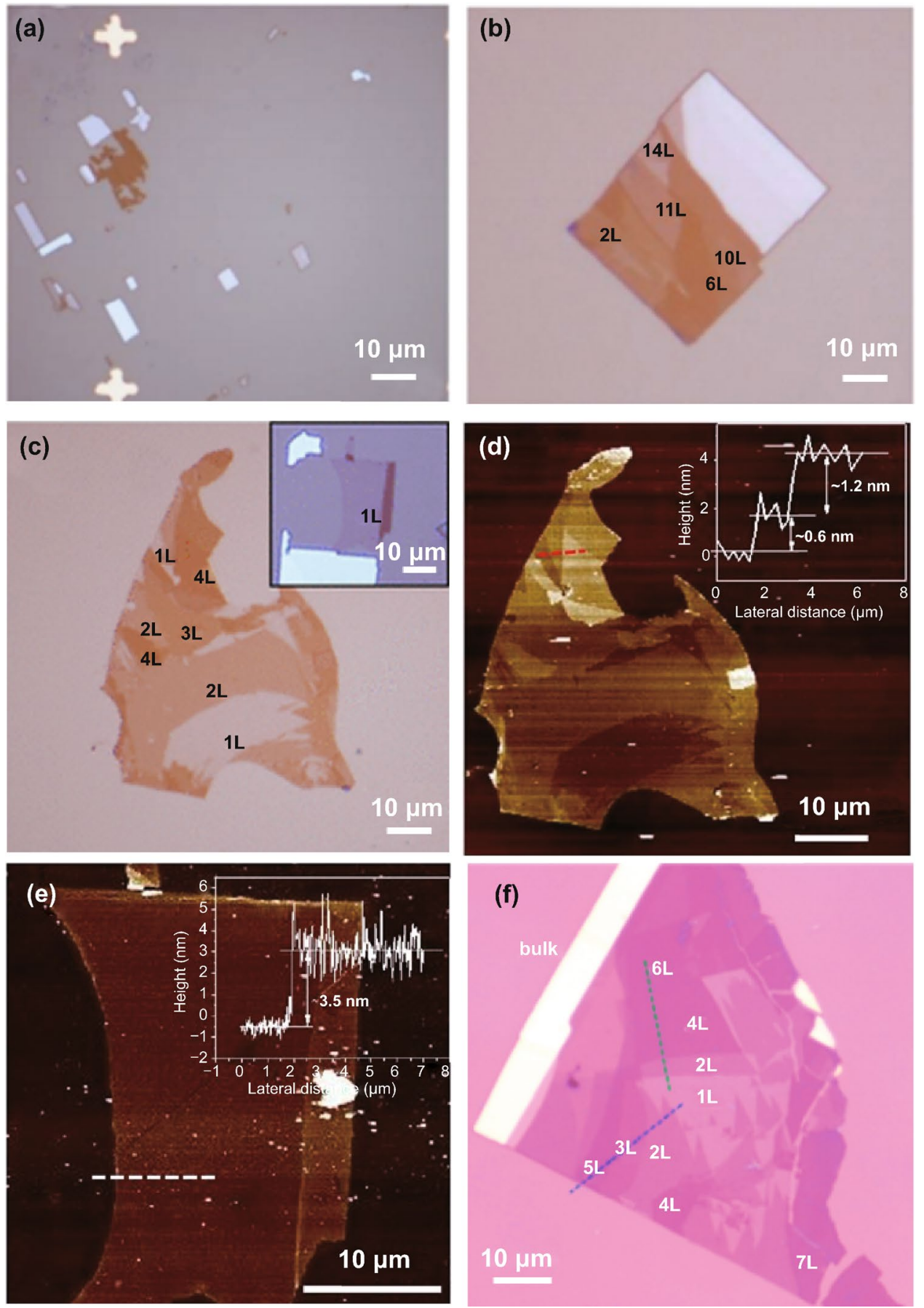

Fig. 11 Mechanically exfoliated $\mathrm{PdSe}_{2}$ flakes. a Optical micrographs of exfoliated $\mathrm{PdSe}_{2}$ nanosheets on the substrate with lithographed metal

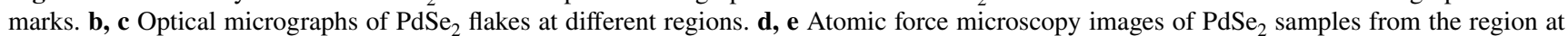
the panel $\mathbf{c}$ and its inset. Reprinted with permission from Ref. [15]. Copyright 2017, American Chemistry Society. f Optical microscopy images of the $\mathrm{PdSe}_{2}$ flakes with different layers. Reprinted with permission from Ref. [39]. Copyright 2020, American Chemistry Society

Here, Se powder was placed in Zone 1 at a temperature of $250{ }^{\circ} \mathrm{C}$, and $\mathrm{PdCl}_{2}$ powder was placed in Zone 2 at a temperature of $500{ }^{\circ} \mathrm{C}$. Then, Se and Pd precursors were transported by $\mathrm{Ar} / \mathrm{H}_{2}$ to Zone 3, and the temperature was maintained at $600{ }^{\circ} \mathrm{C}$, at which the polycrystalline $\mathrm{PdSe}_{2}$ films were synthesized continuously on the substrate. 
Table 3 Different types of $\mathrm{PdSe}_{2}$ from the various synthesis approaches. These scheme were drawn by the authors, which were inspired by the literatures, i.e., bulk [17], flake [42], nanosheet [137], large domain [27] and 2D film [138]

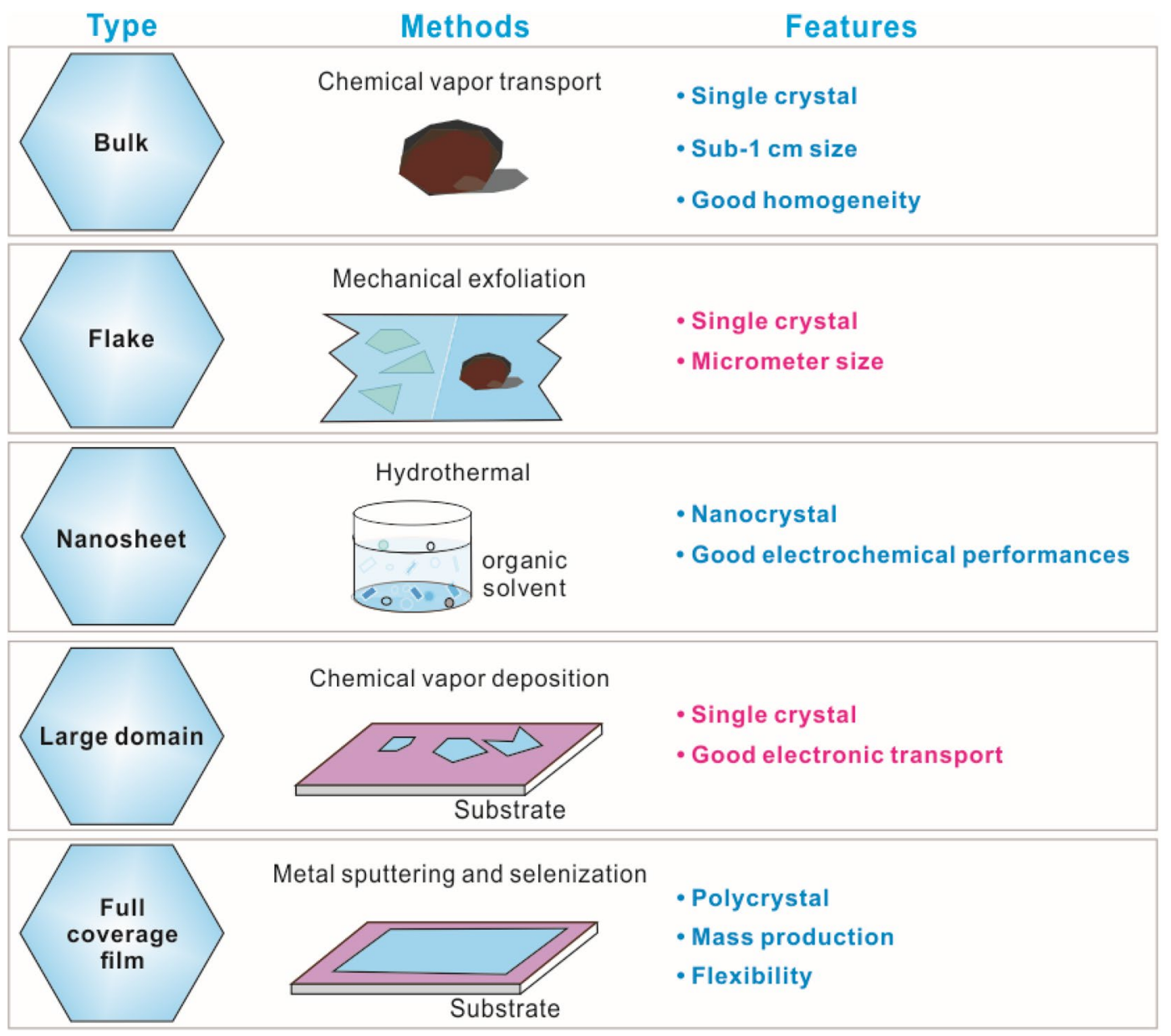

Figure $12 \mathrm{~b}$ shows a photograph of the as-grown $\mathrm{PdSe}_{2}$ film with high uniformity. The AFM image and height profile of the $\mathrm{PdSe}_{2}$ films were characterized (Fig. 12c) with a thickness of $\sim 8 \mathrm{~nm}$, corresponding to 20 layers of $\mathrm{PdSe}_{2}$ [15].

Because of the high melting point of the Pd metal precursor, the molten salt-assisted method can be utilized for the growth of $\mathrm{PdSe}_{2}$ flakes, which can be synthesized at a lower temperature over a large domain [61]. The ambient pressure chemical vapor deposition (APCVD) method can be used with the assistance of salt powder, such as $\mathrm{NaCl}$, where the $\mathrm{Pd}$ metal precursor is replaced by high-purity $\mathrm{PdCl}_{2}$ powder. $\mathrm{Au}$ foils were placed above the mixture and heated at $850-900{ }^{\circ} \mathrm{C}$ at $85 \mathrm{sccm} \mathrm{Ar}$ and $15 \mathrm{sccm} \mathrm{H}_{2}$ flows for 10-15 min. Interestingly, the length/width ratio of the $\mathrm{PdSe}_{2}$ flakes increased markedly during the synthesis. $\mathrm{PdSe}_{2}$ flakes were obtained with growth times of 20 and 35 min, respectively. The $\mathrm{PdSe}_{2}$ flakes on Au foil exhibited a ribbon-like shape, which was rarely the case on the amorphous oxide substrates. Hence, the synthesis of $\mathrm{PdSe}_{2}$ may depend on its anisotropic structure and orthorhombic symmetry.

\subsection{Chemical Vapor Deposition Reaction by the Sublimated Pd and Se}

A CVD approach has been developed with the reaction of sublimated Pd and Se for growing few-layer PdSe ${ }_{2}$ flakes with high crystallinity [62]. In the setup for the synthesis of $\mathrm{PdSe}_{2}$ crystals, the Se powder was placed in a separate quartz tube zone wrapped with a heating belt at $350{ }^{\circ} \mathrm{C}$, while Pd powder was located in the center of the furnace at $800{ }^{\circ} \mathrm{C}$, with an Ar flow of $50-150 \mathrm{sccm}$ for $10-20 \mathrm{~min}$. Meanwhile, the substrate was placed in the downstream zone 

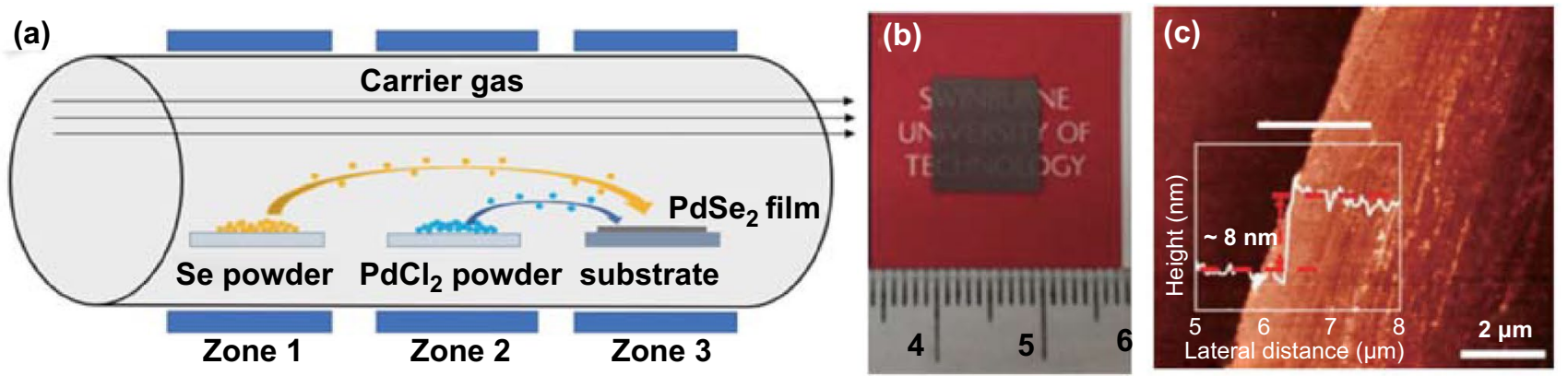

Fig. 12 a Schematic of $\mathrm{PdSe}_{2}$ synthesis process using CVD method. b, c Photograph and AFM height profile of a prepared multilayer PdSe ${ }_{2}$ film. Reprinted with permission from Ref. [60]. Copyright 2020, American Chemical Society

outside the heating zone at $480-600{ }^{\circ} \mathrm{C}$. The scheme of the growth method is presented in Fig. 13.

Notably, the $\mathrm{PdSe}_{2}$ flakes had various thicknesses, sizes, and shapes when the substrates were synthesized at different temperatures. For example, square-like flakes grown at $600{ }^{\circ} \mathrm{C}$ are thicker and larger than the heart-like flakes grown at a temperature of $500{ }^{\circ} \mathrm{C}$.

Chemical vapor deposition has been employed for synthesizing large-area $\mathrm{PdSe}_{2}$ films [16], single-crystal domains [63], nanowires [48], and ribbons [64]. Wafer-scale single-crystal $\mathrm{PdSe}_{2}$ may be necessary for integrated circuit applications.

\subsection{Selenization of Pd Film}

A simple selenization method leads to the synthesis of noble metal diselenide films [65]. The synthesis of $\mathrm{PdSe}_{2}$ films by direct selenization and the thickness of $\mathrm{PdSe}_{2}$ can be well controlled by varying the thickness of the deposited Pd layer [62]. The Pd layer deposited on the substrate via magnetron sputtering was placed in the center zone of the tube furnace at $480{ }^{\circ} \mathrm{C}$, while the high-purity Se powder $(99.99 \%)$ was placed in the upstream zone at $220^{\circ} \mathrm{C}$ under a 60 -sccm Ar flow for $90 \mathrm{~min}$. The selenization strategy could enable the wafer-scale growth of $\mathrm{PdSe}_{2}$, such as in the form of a 2-inch $\mathrm{PdSe}_{2}$ film over a Si wafer [62]. The Raman mapping of the $\mathrm{PdSe}_{2}$ film proves that the $\mathrm{PdSe}_{2}$ film possesses good uniformity.

The structure-property relationship is listed in Table 4. The advantages and disadvantages are compared for different synthesis approaches for obtaining $\mathrm{PdSe}_{2}$. Future opportunities lie in the synthesis of monolayer single-crystal $\mathrm{PdSe}_{2}$ full film over a wafer scale (yet shown).

\subsection{Direct van der Waals Epitaxial Growth of $\mathrm{PdSe}_{2}$ on Graphene}

The PdSe $e_{2}$ has been deposited over the support of graphene or $\mathrm{MoS}_{2}$ in an epitaxial growth fashion [22]. The precursor of Pd containing organic molecules has been employed for the formation of $\mathrm{PdSe}_{2}$. Figure 14a illustrates a schematic of the experimental process. The van der Waals heterostructure of $\mathrm{PgSe}_{2} /$ graphene can be directly grown with this method.

Temperature 1

Temperature 2

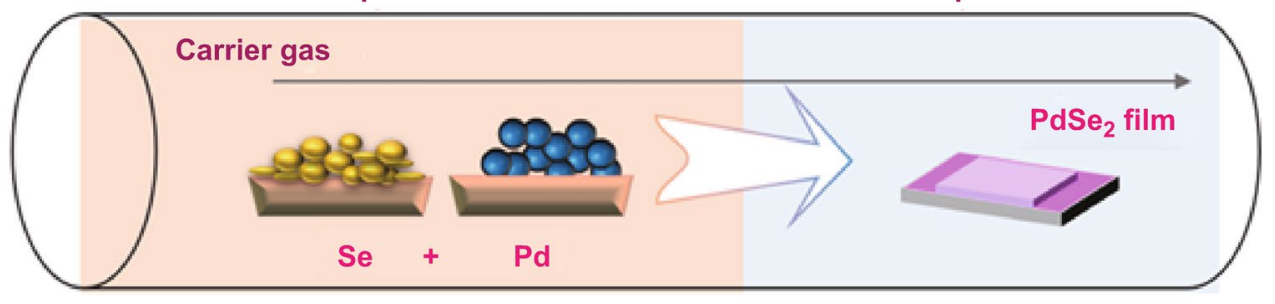

Fig. 13 The chemical vapor deposition synthesis of the $\mathrm{PdSe}_{2}$ film. The Se power and Pd metal are sublimated in temperature 1 and deposited at temperature 2 for $2 \mathrm{D}$ film synthesis. The concept was inspired by Ref. [62]. The scheme is originally drawn by the authors of the review 


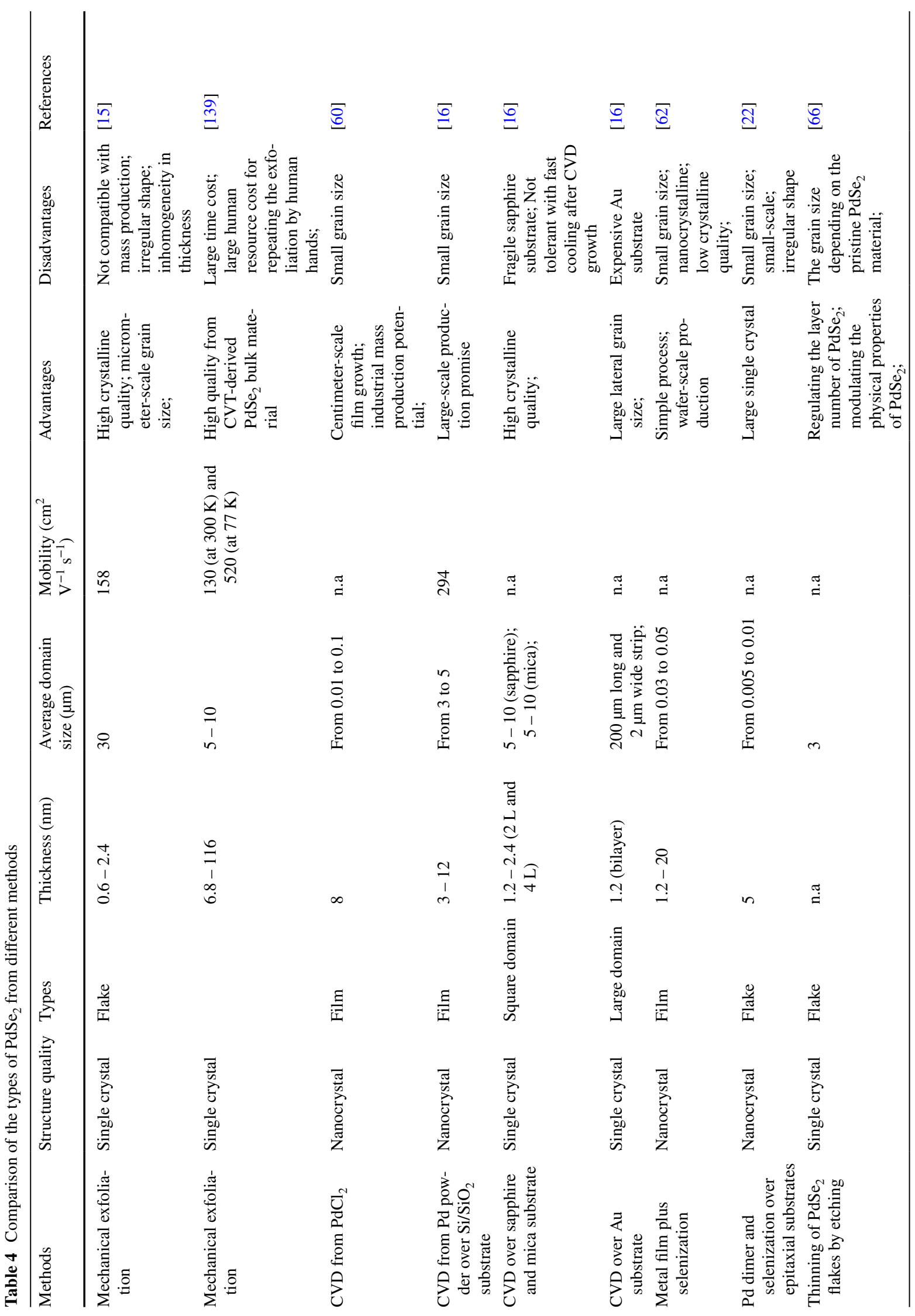


Graphene was suspended on top of the observation membrane by drop-casting the same volume of dispersion onto a TEM grid. The graphene was transferred onto a $0.50 \times 0.50$ $\mathrm{mm}^{2} \mathrm{SiN}_{\mathrm{x}}$ membrane, which has $2-\mu \mathrm{m}$ vacuum pinholes spaced $5 \mu \mathrm{m}$ apart.

Figure 14b shows the CVD system for the selenization of $\mathrm{PdSe}_{2}$. The two-zone furnace was compiled with the temperature profile for Zone 1 at $240{ }^{\circ} \mathrm{C}$ and Zone 2 at $360{ }^{\circ} \mathrm{C}$ (Fig. 14c). This research presents a direct method for the growth of vdWHs at the nanoscale and atomic level and an innovative strategy for the synthesis of 2D materials through predetermined nucleation.

\subsection{Layer-by-layer Thinning by the Oxygen Plasma}

Precise layer control of $\mathrm{PdSe}_{2}$ samples plays an important role in tuning of the bandgap of $\mathrm{PdSe}_{2}$. A layer-by-layer thinning strategy has been employed for etching an $n$-layered $\mathrm{PdSe}_{2}$ flake to the $(n-1)$ layered flake (Fig. 15). Precise layer thinning [66] has been depicted by selective oxidation via oxygen plasma and sublimation through thermal annealing (Fig. 15a-d).

To investigate the etching method, the $\mathrm{PdSe}_{2}$ flakes were exposed to plasma with different $\mathrm{O}_{2} / \mathrm{Ar}$ ratios [66]. Figure $15 \mathrm{e}$ shows the variation in the thickness of the $\mathrm{PdSe}_{2}$ flakes after etching. The correlation between the thickness and number of layers employs an empirical value of $0.7 \mathrm{~nm}$ per $\mathrm{PdSe}_{2}$ layer [15]. Figure $15 \mathrm{f}$ shows an optical micrograph of two pristine $\mathrm{PdSe}_{2}$ flakes with seven and nine layers, respectively. Figure $15 \mathrm{~g}$ shows the same regions after the plasma etch cycle. The color of the $\mathrm{PdSe}_{2}$ species changes subtly from blue to light purple, which indicates a decrease in the $\mathrm{PdSe}_{2}$ film thickness.

The AFM images of the corresponding $\mathrm{PdSe}_{2}$ flakes (Fig. 15h, i) provide line-scanning information (Fig. 15j). Here, 2-nm $\mathrm{PdSe}_{2}$ (ca. 3 layers) was etched after oxidation and sublimation upon $\mathrm{O}_{2}$ plasma treatment. Therefore, plasma etching and surface curing may shed light upon the bandgap regulation of 2D materials over a large area.

The posttreatment of $\mathrm{PdSe}_{2}$ could modify the structure and properties of the pristine material. First, mild plasma exposure to $\mathrm{PdSe}_{2}$ could lead to layer-by-layer plasma etching to regulate the thickness [66]. The ozone treatment [67] 
(a)

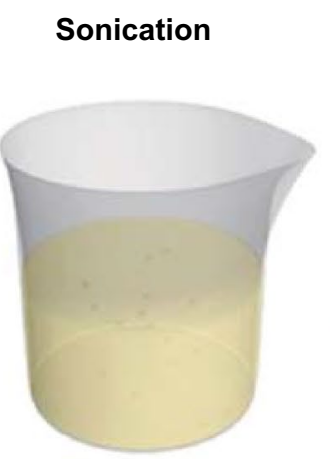

Growth of $\mathrm{PdSe}_{2}$ on graphene

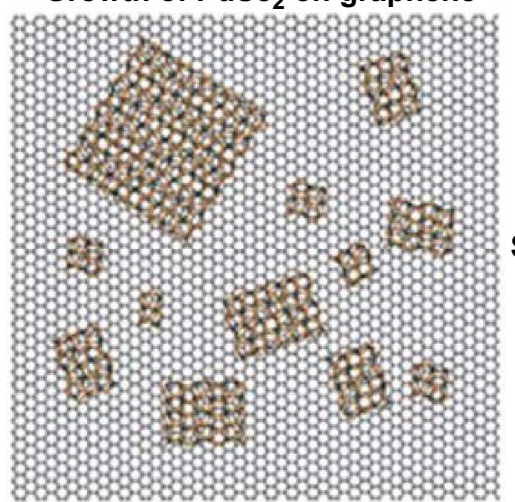

\section{Drop-Casting}
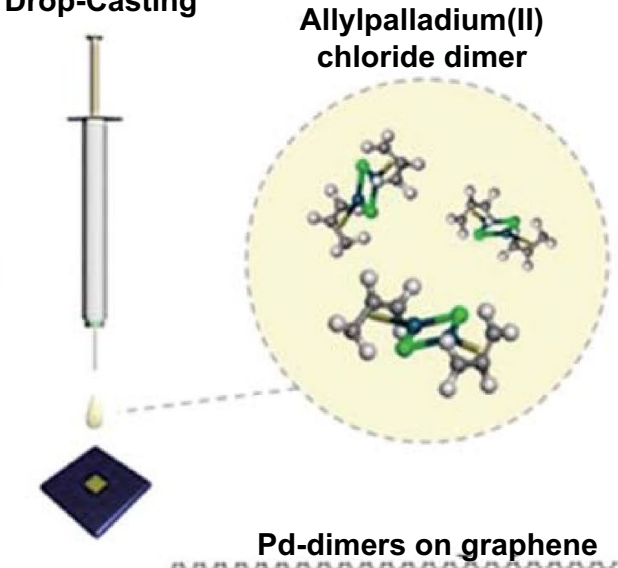

Pd-dimers on graphene

Selenization
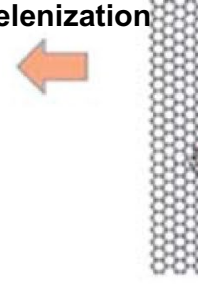

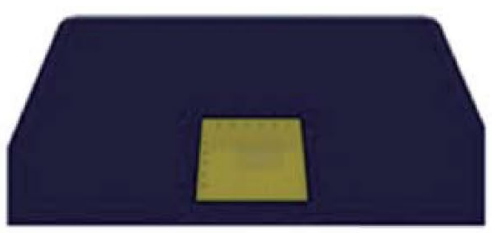

Si (b)

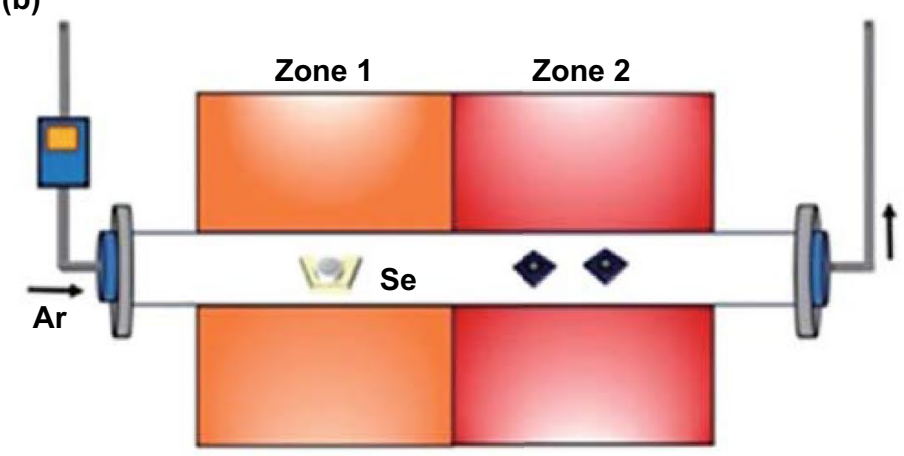

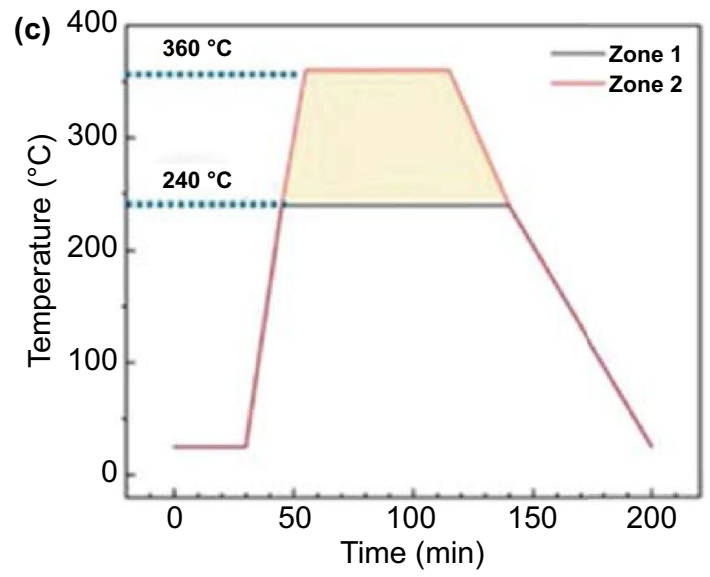

Fig. 14 Growth of $\mathrm{PdSe}_{2}$ over graphene with selenization of Pd dimers as precursors. a Schematic of protocol for PdSe $\mathrm{S}_{2}$ synthesis. b Schematic of two-zone horizontal furnace for thermal treatment under Se-rich atmosphere. c Temperature distribution along with tube furnace. Reprinted with permission from Ref. [22]. Copyright 2020, American Chemical Society

of $\mathrm{PdSe}_{2}$ could enhance the chemical sensitivity owing to the weak oxidation. Electron irradiation can modify conductivity performance [68]. The phase transformation of $\mathrm{PdSe}_{2}$ leads to a sub-1-nm channel by thermal treatment [49] and the $\mathrm{Pd}_{2} \mathrm{Se}_{3}$ phase by interlayer fusion [46].

\section{Roles in Electronic Devices}

As mentioned above, because of the strong interlayer interactions resulting from the almost fully occupied $d$-orbital and tunable properties, which depend on the number of 


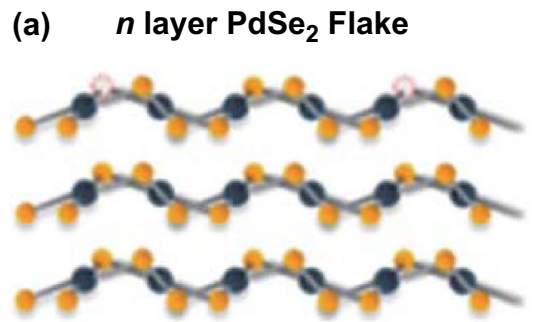

Palladium

Selenium

Oxygen

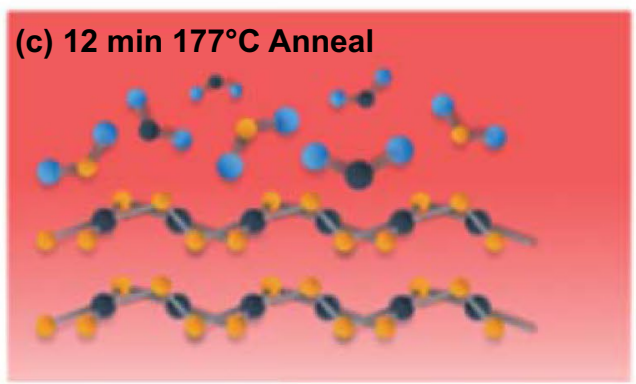

(e)

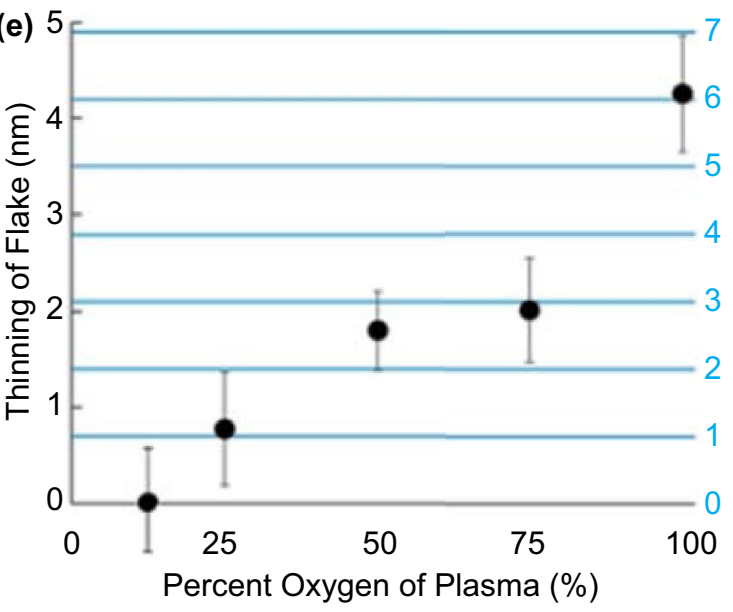

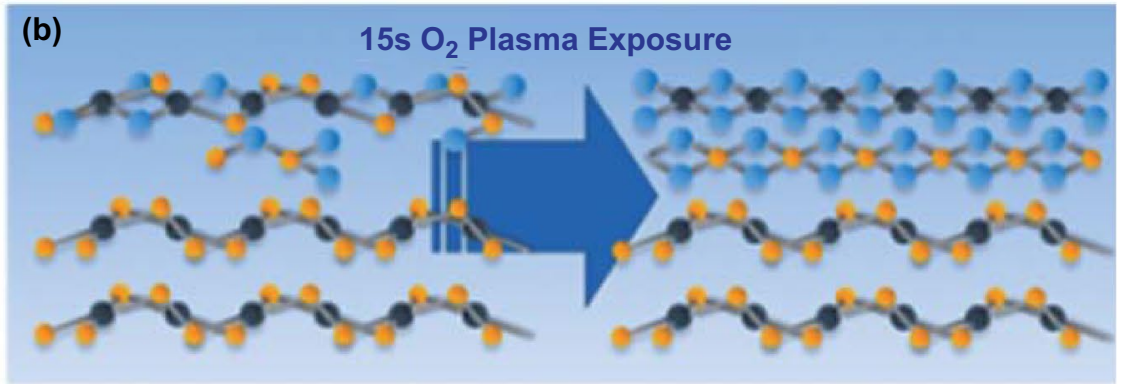

(d) n-1 layer $\mathrm{PdSe}_{2}$ Flake (f)
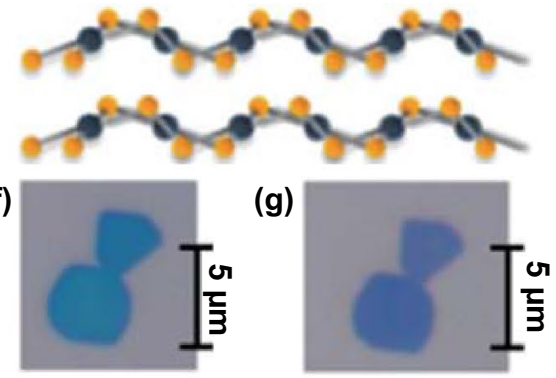

(g)

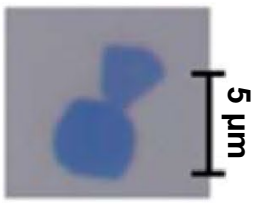

(h)

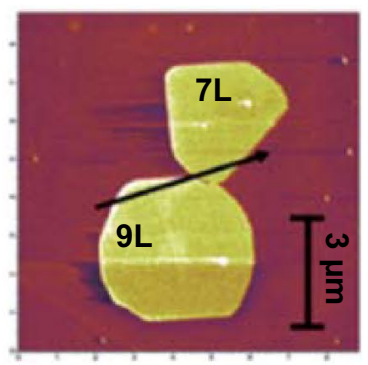

(i)

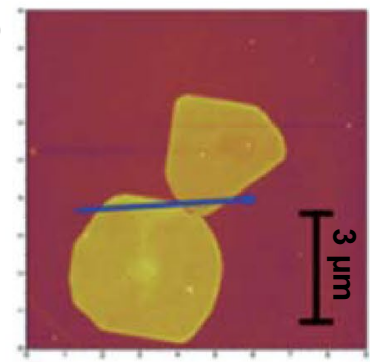

(j)

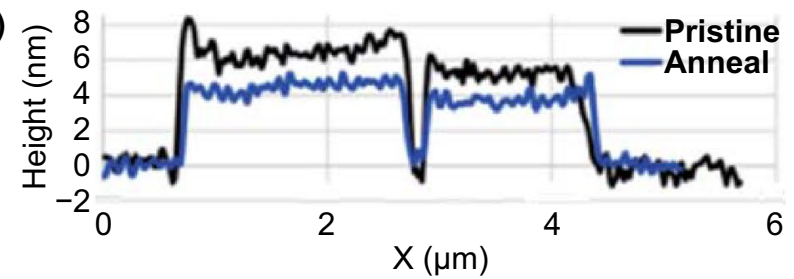

Fig. 15 Thinning of $\mathrm{PdSe}_{2}$ layers with plasma treatment. a Pristine PdSe $e_{2}$ flakes. b Oxygen plasma etching. c Thermal annealing. d Resultant $\mathrm{PdSe}_{2}$ after layer thinning. e Etching of layers versus the oxygen percentage in the plasma. Optical micrographs of PdSe $\mathrm{P}_{2} \mathbf{f}$ before plasma etching and $\mathbf{g}$ after plasma thinning. Atomic force microscope micrographs of $\mathrm{PdSe}_{2}$ flakes before $\mathbf{h}$ and after $\mathbf{i}$ layer thinning. $\mathbf{j}$ Height profiles from two lines extracted from panel $\mathbf{h}$ and panel i. Reprinted with permission from Ref. [66]. Copyright 2020, American Chemical Society

layers, $\mathrm{PdSe}_{2}$ shows potential as a $2 \mathrm{D}$ material applicable for use in electronic devices.

\subsection{Electrical Contacts for $\mathrm{PdSe}_{2}$ Devices}

Prior to fabrication of an electric device, a metal/PdSe $\mathrm{P}_{2}$ contact is essential for optimizing the electrical performance of transistors, photodetectors, and integrated circuits. At the interface of metal/semiconductor contact, the transport properties of charge carriers are determined by the Schottky height, tunneling energy barrier, orbital overlapping percentage, as well as the geometry of the interface.

Theoretical calculations using the DFT approach were employed to compare the metal/PdSe ${ }_{2}$ contact performances by tuning the metal types such as $\mathrm{Au}, \mathrm{Ag}, \mathrm{Pb}, \mathrm{Cu}$, and $\mathrm{Ti}$, as well as semimetallic graphene. The efficiency of 
charge transfer at the $\mathrm{PdSe}_{2}-$ metal interface was examined for energy barrier evaluation [69]. Figure 16a shows the prototype of a $\mathrm{PdSe}_{2}-$ metal contact with a carrier flowing from the metal electrode to the $\mathrm{PdSe}_{2}$ channel through the pathway $(\mathrm{I} \rightarrow \mathrm{II} \rightarrow \mathrm{III} \rightarrow \mathrm{IV} \rightarrow \mathrm{V})$. In a typical $\mathrm{PdSe}_{2}$ FET (Fig. 16b), carriers diffuse from the metal to the layered $\mathrm{PdSe}_{2}$ and encounter a tunneling barrier, which depends on the binding strength at the interface of the $\mathrm{PdSe}_{2}$-metal contact.

The $\mathrm{PdSe}_{2}-$ metal contacts, i.e., with $\mathrm{Au}$ or $\mathrm{Ag}$ electrodes, are demonstrated with their energy band alignment based on the binding energy owing to the Schottky barriers (Fig. 16c). First, $\mathrm{Au} / \mathrm{PdSe}_{2}$ was preferred via compression of the Schottky barrier height. Meanwhile, the Ag electrodes led to an improved orbital overlap with $\mathrm{PdSe}_{2}$. A vertical Schottky barrier appears at the interface (II) in the vertical direction, while a lateral Schottky barrier occurs at the interface (IV) between the heterojunction and the $\mathrm{PdSe}_{2}$ channel region. Second, the $\mathrm{Pb} / \mathrm{PdSe}{ }_{2}$ contact has a low tunneling potential with a Schottky barrier height of $0.67 \mathrm{eV}$ (Fig. 16d). Third, $\mathrm{Cu} / \mathrm{PdSe}_{2}$ does not form a tunneling interface (Fig. 16e) but has a Schottky height of $0.58 \mathrm{eV}$. (a)

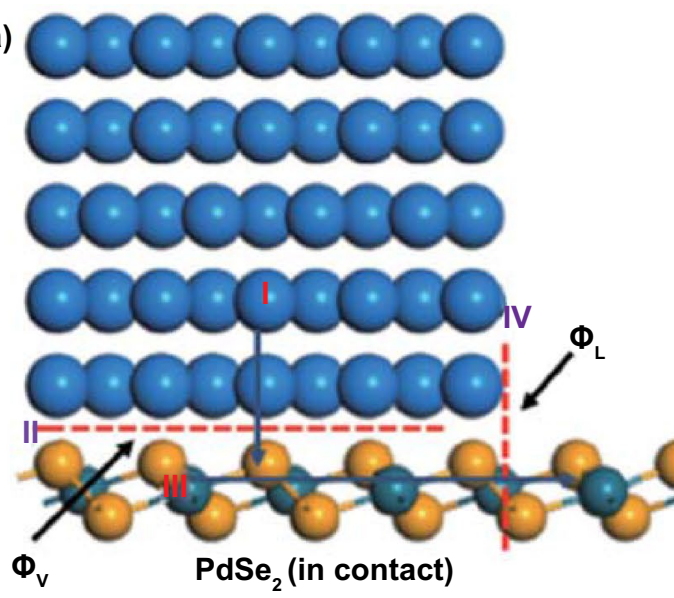

(b)

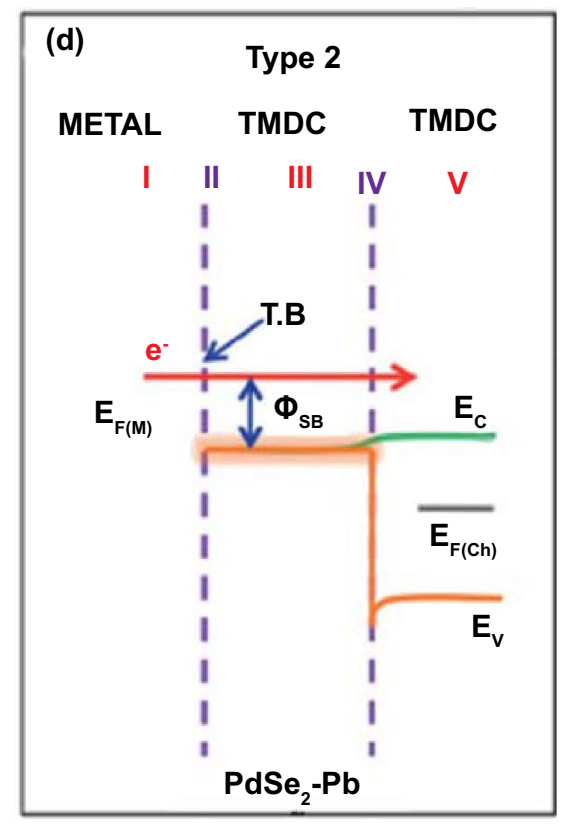

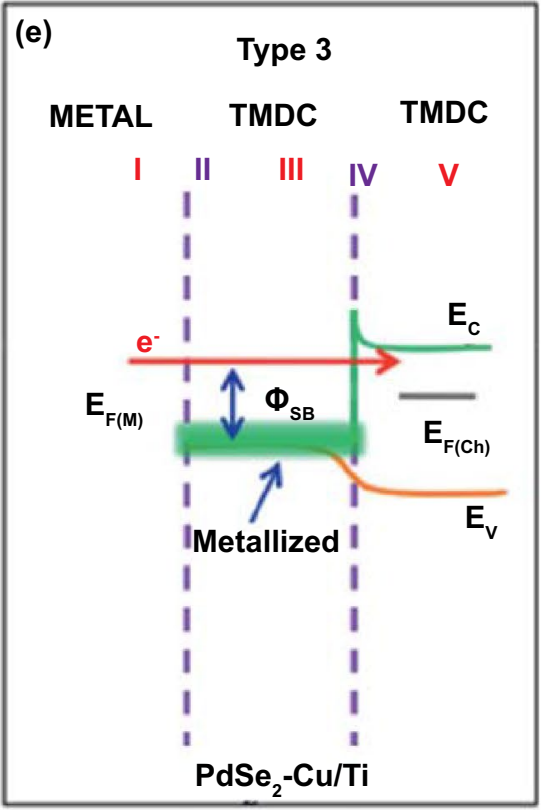
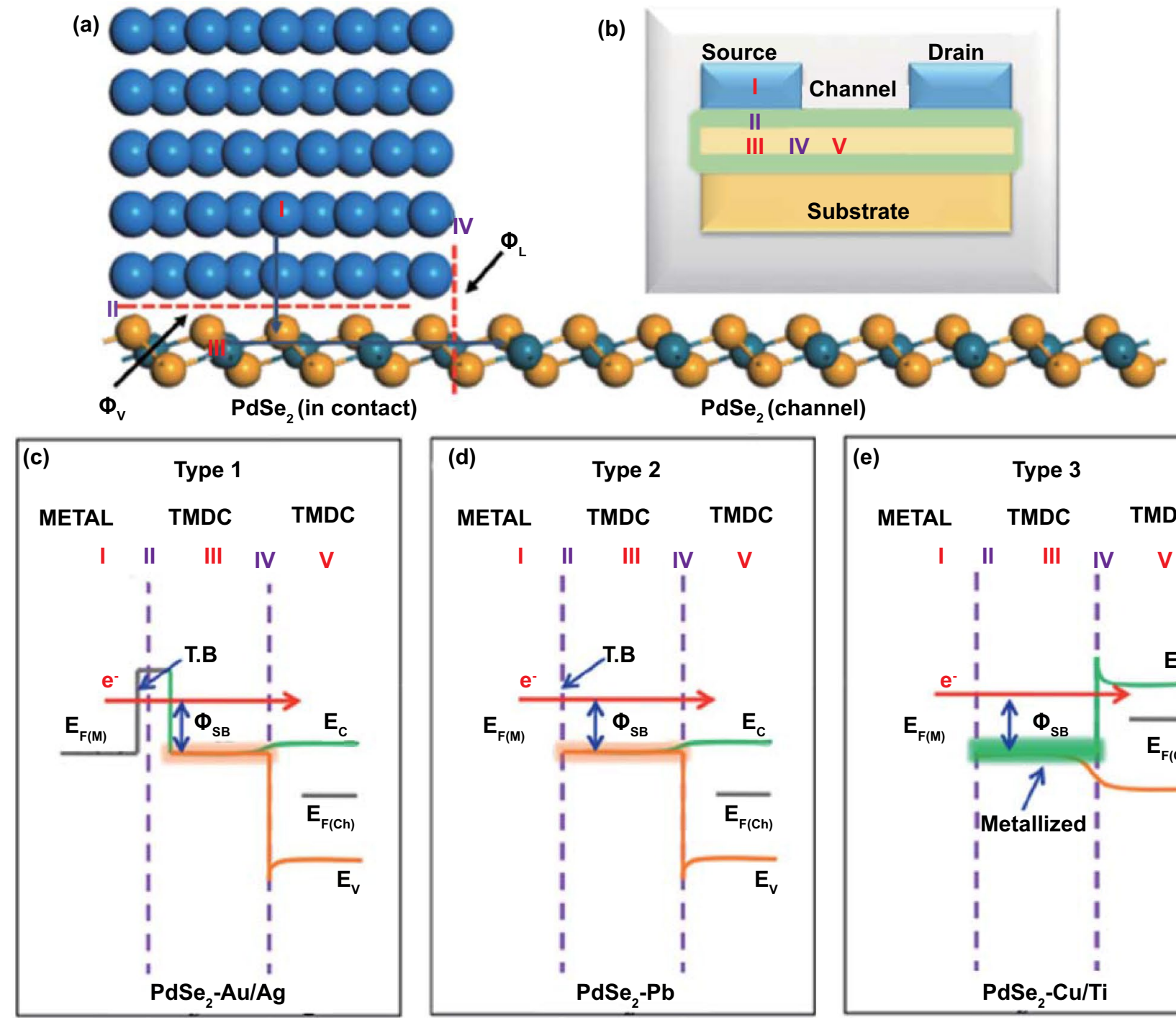

Fig. $16 \mathrm{Metal} / \mathrm{PdSe}_{2}$ contact, transistor configuration, and their band alignment. a Atomic configuration of PdSe $\mathrm{e}_{2}$-metal contact. The pathway of electron flows is coordinated from the metal electrode to the metal/ $\mathrm{PdSe}_{2}$ interface and then to the $\mathrm{PdSe}_{2}$ channel. b Schematic of typical PdSe ${ }_{2}$ field-effect transistor. The labeling is identical for the five panels of I, II, III, to IV and V. Energy band alignment of different PdSe ${ }_{2}-\mathrm{metal}_{\text {con- }}$ tacts based on tunneling evaluation and Schottky barriers with $\mathbf{c}$ weak bonding by Au/Ag, $\mathbf{d}$ medium bonding with $\mathrm{Pb}$, and $\mathbf{e}$ strong bonding by $\mathrm{Cu}$ /Ti interface. "T.B." denotes the tunneling barrier. Reprinted with permission from Ref. [69]. Copyright 2020, American Chemical Society 
Eventually, the graphene/ $\mathrm{PdSe}_{2}$ contact has emerged as a proof of concept with regard to vdWHs. A Schottky barrier height of $0.22 \mathrm{eV}$ is preferred for electron transport-that is, $n$-type charge carrier conductance [69]. Indeed, electrons are transferred from the interface to the $\mathrm{PdSe}_{2}$ side with a band bending of -0.94 . The weak van der Waals interactions between graphene and $\mathrm{PdSe}_{2}$ render a quasi-Ohmic contact without energy transfer between the two surfaces. That is, the intrinsic transport properties of $\mathrm{PdSe}_{2}$ are maintained. Analogous to the contact behavior of other 2D materials, one can fabricate high-performance optoelectronic devices.

The stability and metallicity of the $\mathrm{Pd}_{17} \mathrm{Se}_{15}$ phase make it an ideal buffering material between the metal and $\mathrm{PdSe}_{2}$. The low lattice mismatch between both palladium selenides guarantees quasi-Ohmic conductance behavior, which suppresses the Schottky barrier height. In contrast, $\mathrm{PdSe}_{2}$ devices with $\mathrm{Pd}_{17} \mathrm{Se}_{15}$ contacts performed better than those with $\mathrm{Ti} / \mathrm{Au}$ contacts [52]. Figure 17a shows the temperature-dependent mobility of $\mathrm{PdSe}_{2}$ devices with $\mathrm{Pd}_{17} \mathrm{Se}_{15}$ contacts and $\mathrm{Ti} / \mathrm{Au}$ contacts, which are approximately 170 and $8 \mathrm{~cm}^{2} \mathrm{~V}^{-1} \mathrm{~s}^{-1}$, respectively.

Figure 17b shows the relationship between the slope and different drain voltages at zero gate voltage. The Schottky barrier height $\Phi_{\mathrm{B}}$ of the Ti/Au contact device $(96.2 \mathrm{meV})$ is greater than that of the $\mathrm{Pd}_{17} \mathrm{Se}_{15}$ contact device $(3.26 \mathrm{meV}$ ). Figure $17 \mathrm{c}, \mathrm{d}$ illustrates the electrode contact of the $\mathrm{PdSe}_{2}$ devices from the Schottky barrier of Ti/Au contacts to the Ohmic contact of $\mathrm{Pd}_{17} \mathrm{Se}_{15}$ contacts. Thus, the contact resistance of the $\mathrm{PdSe}_{2}$ device decreases and has the potential to become closer to the quantum limit.

Future opportunities may remain in developing the electrical contacts of $\mathrm{PdSe}_{2}$ with other palladium selenides. Indeed, the $\mathrm{PdSe}_{2-x}$ phases with different stoichiometric ratio may arouse different contact behaviors when stacking vertically with $\mathrm{PdSe}_{2}$ or stitching together laterally. The $\mathrm{PdSe}_{2-x} / \mathrm{PdSe}_{2}$ contact could be either Ohmic or Schottky
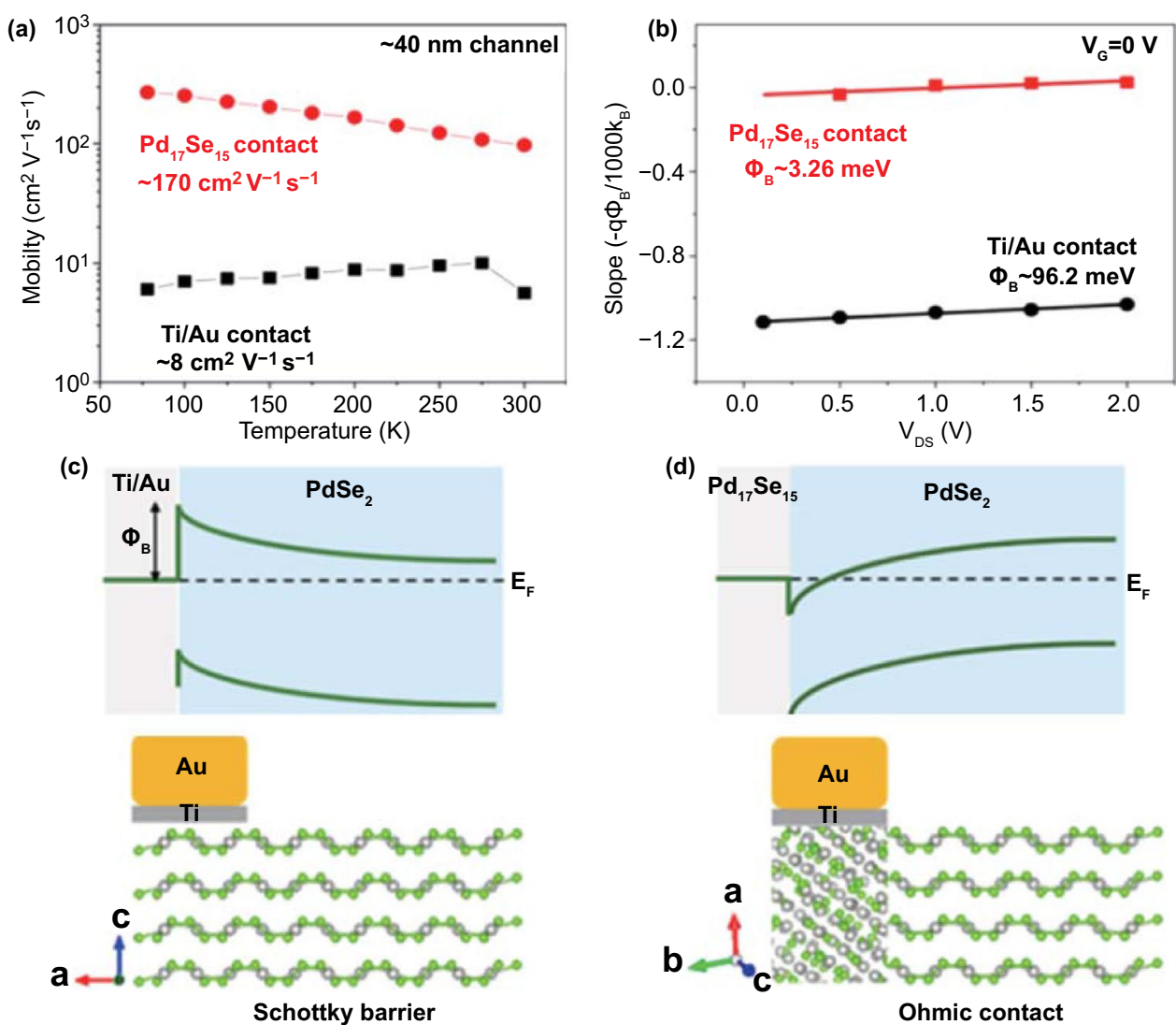

Fig. $17 \mathrm{Metal} / \mathrm{PdSe}_{2}$ contact for regulating the electronic transports. a Comparison of temperature-dependent mobility of the PdSe ${ }_{2}$ channel with Ti/Au and $\mathrm{Pd}_{17} \mathrm{Se}_{15}$ contacts, respectively. b Comparison of $\mathrm{V}_{\mathrm{DS}}$-dependent Schottky barrier height of PdSe 2 devices with Ti/Au and $\mathrm{Pd}_{17} \mathrm{Se}_{15}$ contacts, respectively. Schematic of $\mathbf{c} \mathrm{Ti} / \mathrm{Au}$ contact and $\mathbf{d} \mathrm{Pd}_{17} \mathrm{Se}_{15}$ contact. Reprinted with permission from Ref. [52]. Copyright 2019, American Chemistry Society 
typed, which require the optimization of researchers. The Schottky type contact could be utilized in the rectifier device. The Ohmic contact facilitates the electronic transport performances such as charge carrier mobility. The phase-engineering method proves that new crystalline phases of anisotropic 2D materials can be induced by defects. These new $\mathrm{PdSe}_{2-x}$ compounds may have different stoichiometries, which broadens the choices of materials for electrical contacts.

After understanding the metal/PdSe $\mathrm{Pe}_{2}$ contact, we now come to the discussion of electronic transport performances in field-effect transistors.

\section{2 $\mathrm{PdSe}_{2}$ Field-effect Transistors}

The field-effect transistors are one of the most significant devices in semiconductor electronics, and FETs based on 2D materials have shown superior performance to those based on traditional semiconductors. Moreover, usage of 2D materials provides new opportunities and effective approaches regarding FETs, with a high on/off ratio, high carrier mobility, and excellent stability. The layer-dependent properties of TMDCs are important for the design of FETs for diverse functionalized devices [30]. In this section, $\mathrm{PdSe}_{2}$ FETs and efficient methods to improve their performance are introduced.

$\mathrm{PdSe}_{2}$ has proven to be a successful channel material for FETs. PdSe $e_{2}$ transistors have achieved high mobility with tunable ambipolar characteristics [70]. Figure 18a shows an experimental setup to measure the $\mathrm{PdSe}_{2}$ FET characteristics with $p$-type $\mathrm{Si}$ as a universal back-gate electrode, and Fig. 18b, c shows SEM images of the two as-fabricated $\mathrm{PdSe}_{2}$ FET samples.

The electronic performances of the $\mathrm{PdSe}_{2}$ FETs are depicted in the output and transfer characteristics (Fig. 18d, e). In the output curves, the $\mathrm{PdSe}_{2}$ FET exhibited a linear correlation between the voltage and current (insets of Fig. 18d, e). Such a linear drain current-voltage dependence indicates quasi-Ohmic contact, i.e., suppression of the Schottky barrier between $\mathrm{PdSe}{ }_{2}$ and the electrodes. The electrons dominate the primary charge carriers of the FET at a positive gate voltage and a small negative gate voltage (Fig. 18f). In contrast, the holes become the leading charge carriers of the FET at a large negative gate voltage.

The air stability of $\mathrm{PdSe}_{2}$ guarantees the lifetime of its transistor-based sensor applications in a wet environment.
For example, the 2D material-based transistors have been embedded in a microfluidic chip for microRNA detection and screening [71]. The chemical sensors of 2D materials have demonstrated superior performances.

The slight hysteresis of the transfer curve could result from the effect of slow trap states and the surface adsorbates from the lithography-based fabrication process [17]. Remarkably, the ambipolar behavior of the $\mathrm{PdSe}_{2}$ FET could be regulated through a biased sweep.

The hysteresis width can be periodically evaluated by continuously measuring several transfer curves. These two transfer curves of the $\mathrm{PdSe}_{2}$ FET were measured in succession (Fig. 18g). The first curve in black was employed as a reference curve, and the second curve in red was collected at a delay of $8 \mathrm{~s}$ after the first signal capture. Therefore, the hysteresis is elevated with the increased sweeping time of the gate voltage.

The transport properties of $\mathrm{PdSe}_{2}$ FETs can be regulated by employing vacuum annealing, charge doping, electrical stress, pressure, and electron irradiation [17]. Through reduction of both the pressure and electron irradiation, hysteresis in the $\mathrm{PdSe}_{2}$ FET can be effectively suppressed. These strategies offer viable methods to reduce hysteresis in devices. Moreover, the types of charge carriers can be converted from $n$-type to $p$-type, which can be used as a switch for practical circuits.

Remarkably, vacuum annealing is an effective method for improving the mobility of FETs, and it has been widely used in electronic devices [72]. For PdSe ${ }_{2}$ FETs, annealing can remove the surface adsorbates to achieve higher mobility, on/off ratio, and lower Schottky barrier. Moreover, annealing at $450 \mathrm{~K}$ can eliminate hysteresis in FETs [17]. After annealing at 400 and $450 \mathrm{~K}, \mathrm{PdSe}_{2}$ FET exhibits a higher mobility of 75 and $216 \mathrm{~cm}^{2} \mathrm{~V}^{-1} \mathrm{~s}^{-1}$ than that measured at room temperature. Such a transistor achieves the highest current ON/OFF ratio of $10^{3}$ at $450 \mathrm{~K}$. Meanwhile, the threshold voltage shows an increasing shift to the negative gate voltage as the annealing temperature increases. This indicates that the Fermi level moved to the conduction band in $\mathrm{PdSe}_{2}$. Thus, the $\mathrm{PdSe}_{2}$ FET exhibits an obvious $n$-type transfer characteristic.

Furthermore, a molecular doping method converts the electron transport behavior of $\mathrm{PdSe}_{2}$ into a hole-transport feature [17]. As a prevalent $p$-dopant, F4-TCNQ has high electron affinity and has been utilized in low-dimensional materials 
(a)

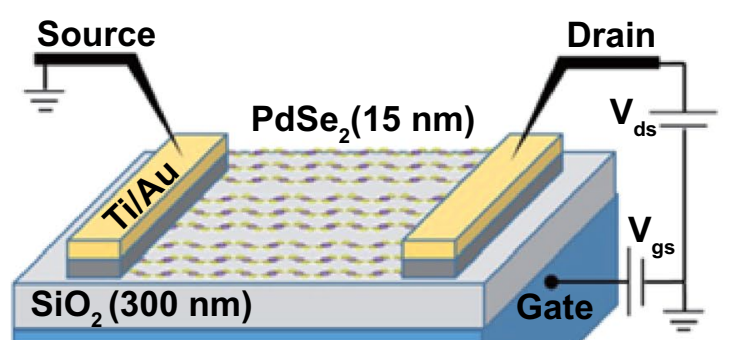

p-type Si
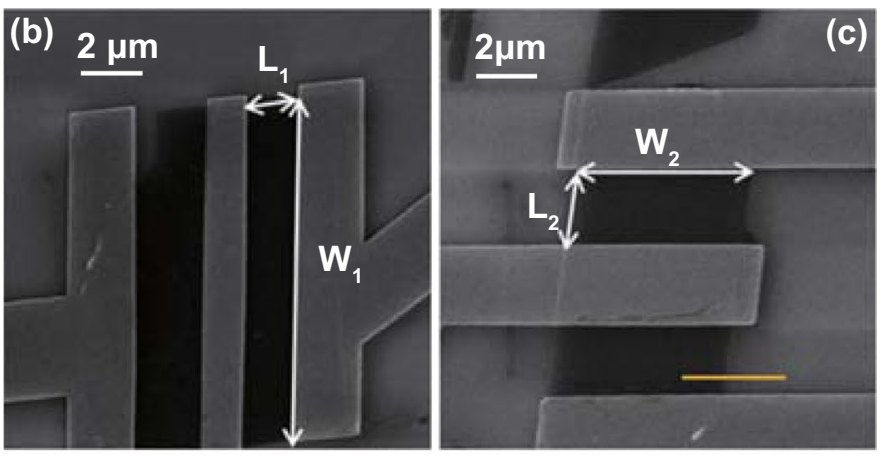

(d)
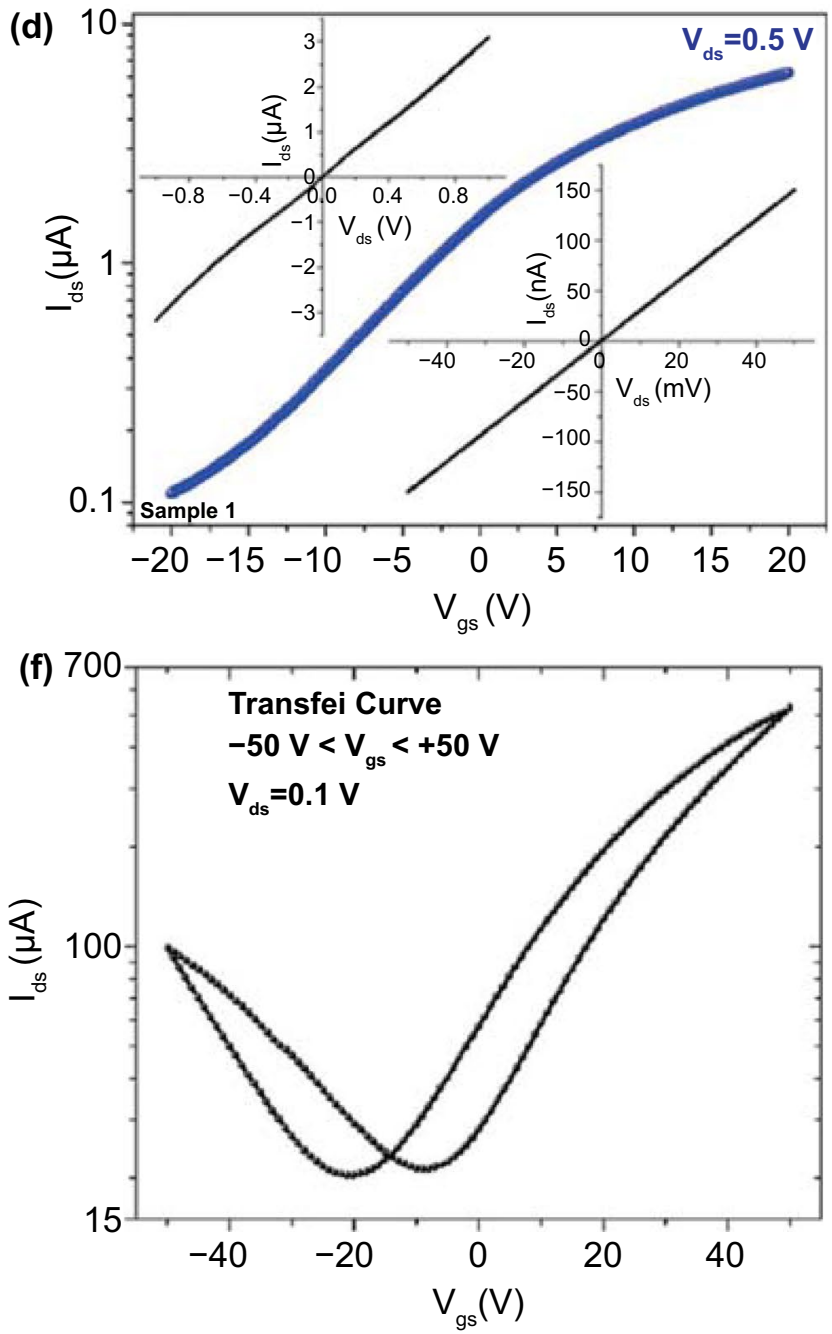
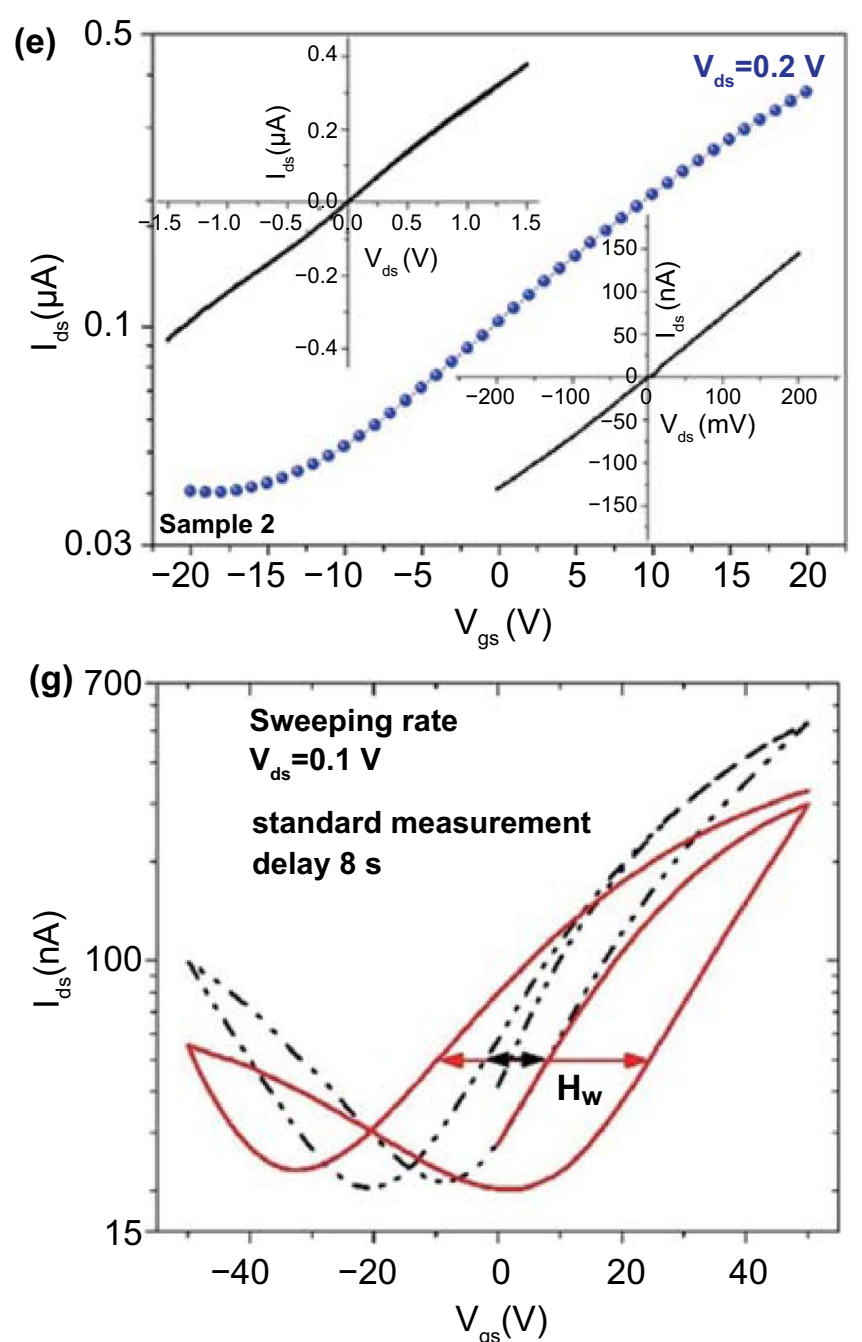

Fig. 18 Demonstration and performance of PdSe ${ }_{2}$ FET. a Schematic of PdSe $e_{2}$ field-effect transistor and electrical measurements. b, c SEM micrographs of two devices with source and drain electrodes fabricated on 15-nm-thick PdSe ${ }_{2}$ flakes. Channel length and width vary in both transistors. d, e Transfer characteristics of $\mathrm{PdSe}_{2}$ FETs corresponding to b, $\mathbf{c}$. Insets are the drain current versus voltage, i. e., the output characteristics of the $\mathrm{PdSe}_{2}$ transistor at the high bias voltage (top left) and low bias voltage (bottom right). f The transfer curves of the PdSe $e_{2}$ FETs measured at vacuum conditions of $10^{-6}$ mbar. $\mathbf{g}$ Comparison of the transfer curves in panel $\mathbf{f}$ with the curves measured after a delay of $8 \mathrm{~s}$ relative to the standard measurement time in panel $\mathbf{f}$. The Hw denotes the hysteresis width. Reprinted with permission from Ref.[74]. Copyright 2019, Elsevier Ltd 
[73], which can be applied to $\mathrm{PdSe}_{2}$. One can compare the transfer curves of the FET with different doping levels from undoped to completely doped, whereby a distinct conversion of the transfer characteristics from ambipolar to $p$-type is shown. The contact resistance of the $\mathrm{PdSe}_{2}$ FET has a dependence on the gate voltage, resulting from the Fermi level being adjusted by electrostatic gating. Therefore, vacuum annealing and molecular doping can effectively reduce contact resistance.

The transport properties of $\mathrm{PdSe}_{2}$ FETs can be altered via annealing or charge doping. Besides, the ambipolar behavior of $\mathrm{PdSe}_{2}$ FETs can be obtained by varying the electrical stress, pressure, and electron irradiation [70].

Atmospheric pressure has a significant influence on the $\mathrm{PdSe}_{2}$ FET. The transfer curves of the $\mathrm{PdSe}_{2}$ FET were extracted under various pressures at a constant drain voltage of $100 \mathrm{mV}$. As the pressure increases, the transfer nature of the $\mathrm{PdSe}_{2}$ FET gradually transforms from the $n$-type to the $p$-type, and the $\mathrm{PdSe}_{2}$ FET exposed to air after $10 \mathrm{~min}$ becomes a $p$-type depletion mode transistor [70].

Moreover, electron irradiation changes the charge distribution in the $\mathrm{PdSe}_{2}$ FET, which further affects the transfer characteristics [74]. The transfer curves of the $\mathrm{PdSe}_{2}$ FETs were collected before and after electron irradiation via SEM imaging. With increasing time after SEM imaging, the transfer characteristics slowly revert to the initial state.

Two-dimensional $\mathrm{PdSe}_{2}$ synthesized using different approaches has been used in the fabrication of FETs.
Table 5 compares the performance of these $\mathrm{PdSe}_{2}$ FETs in terms of charge carrier mobility and current $\mathrm{ON} / \mathrm{OFF}$ ratio. Further developments with large-area CVD-grown $\mathrm{PdSe}_{2}$ may improve the electrical performance, such as the charge mobility and ON/OFF ratio.

In summary, several strategies have been developed to improve the FET performance of $\mathrm{PdSe}_{2}$. Future opportunities still exist in terms of surface cleaning and modification, electrode contact design, packaging conditions, and vdWH stacking. Indeed, the $\mathrm{PdSe}_{2}$-based electronic devices could be integrated with the piezoelectric materials, i.e., PVDF for tactile sensors [75]. For the comfort of human beings, stretchable and wearable electronics become emerging with device development such as strain sensors and electronic skin [76]. Besides, the introduction of triboelectric nanogenerators, supercapacitors [77], and batteries [78] may lead to self-powered sensors [79].

After knowing the electronic devices of $\mathrm{PdSe}_{2}$, we turn to the progress in its applications in optoelectronics and optics.

\section{$5 \mathrm{PdSe}_{2}$ for Optoelectronics and Optics}

The photodetector, which is a device that converts an optical signal into an electrical signal instantaneously, plays an indispensable role in current and burgeoning technology, in

Table 5 The performances of field-effect transistors based on $\mathrm{PdSe}_{2}$ obtained from different methods

\begin{tabular}{|c|c|c|c|c|}
\hline $\begin{array}{l}\text { Synthesis methods and } \mathrm{PdSe}_{2} \\
\text { types }\end{array}$ & Electrode types & Charge mobility $\left(\mathrm{cm}^{2} \mathrm{~V}^{-1} \mathrm{~s}^{-1}\right)$ & Current ON/OFF ratio & References \\
\hline CVD (Domains) & $\mathrm{Cr} / \mathrm{Au}$ & 294 & $10^{3}$ & {$[16]$} \\
\hline Exfoliated (flake) & $\mathrm{Ti} / \mathrm{Au}$ & 158 & $10^{6}$ & {$[15]$} \\
\hline CVD (Domains) & $\mathrm{Ti} / \mathrm{Au}$ & 6.4 & $>10^{6}$ & {$[63]$} \\
\hline Exfoliated (flake) & $\mathrm{Ti} / \mathrm{Au}$ & 4 & $10^{4}$ & [19] \\
\hline Exfoliated (flake) & $\mathrm{Pd} / \mathrm{Au}$ & 20 & $10^{2}$ & {$[81]$} \\
\hline Exfoliated (flake) & $\mathrm{Pd}_{17} \mathrm{Se}_{15} / \mathrm{Ti} / \mathrm{Au}$ & 170 & n.a & {$[52]$} \\
\hline Exfoliated (flake) & $\mathrm{Ti} / \mathrm{Au}$ & 8 & n.a & {$[52]$} \\
\hline Exfoliated (flake) & $\mathrm{Ti} / \mathrm{Au}$ & 216 & $10^{3}$ & {$[17]$} \\
\hline CVD (domains) & $\mathrm{Cr} / \mathrm{Au}$ & n.a & $10^{3}$ & [140] \\
\hline Exfoliated (flake) & $\mathrm{Ti} / \mathrm{Au}$ & 4 & 25 & {$[70]$} \\
\hline Exfoliated (flake) & $\mathrm{Ti} / \mathrm{Au}$ & 3 & 30 & {$[74]$} \\
\hline Exfoliated (flake) & $\mathrm{Ti} / \mathrm{Au}$ & 92 & $10^{4}$ & {$[41]$} \\
\hline Exfoliated (flake) & $\mathrm{Ti} / \mathrm{Au}$ & 138.9 & $10^{3}$ & {$[54]$} \\
\hline
\end{tabular}


the fields of biotechnology, medicine, physics, and natural sciences [80].

Owing to their unique and significant properties, 2D materials have been applied in photodetectors and exhibit remarkable performance in terms of responsivity $(\mathrm{R})$, detectivity $\left(D^{*}\right)$, and external quantum efficiency (EQE) [81]. Here, the responsivity $R$ describes the photoelectric conversion efficiency, $D^{*}$ reflects the ability to measure the minimum optical signal, and EQE is the ratio of the number of photo-generated electron-hole pairs contributing to photocurrent to the number of the incident photons. The rise/fall time is a crucial parameter for evaluating the response speed of photodetectors.

Two-dimensional materials can be used as outstanding photodetector components by constructing heterojunctions [82] and gate-voltage regulated phototransistors [41]. For example, infrared photodetectors can employ the sensing materials such as BP [83], $\mathrm{PtTe}_{2}$ [10], and $\mathrm{WS}_{2}$. But h-BN, graphene/Si [84], and $\mathrm{MoS}_{2} / \mathrm{GaN}$ [85] can be used for ultraviolet light detection. Besides, $\mathrm{PtSe}_{2}$ has a large photoresponse at a wide spectral band ranging from 200 to $1550 \mathrm{~nm}$ [7]. Then, the anisotropic compounds such as $\mathrm{PdSe}_{2}$ can be used for polarized sensitive photoelectric detection [29]. Therefore, the coupling of $\mathrm{PdSe}_{2}$ and other 2D materials may cover the light detection of a broad spectral range.

In this section, we will discuss the detection band versus bandgap, photodetection performances, and polarized light detection based on $\mathrm{PdSe}_{2}$ and related materials.

\subsection{Detection Bands versus Bandgap}

The performance of the photodetectors can be determined by the bandgaps of the materials. Photodetectors function at various wavelengths based on different 2D materials. Owing to the different bandgaps of the 2D materials, the corresponding photodetectors function in different spectral bands (Table 6).

The performances of 2D material-based photodetectors can be determined as per details, such as black phosphorene or black phosphorus, $\mathrm{MoS}_{2}, \mathrm{MoSe}_{2}, \mathrm{WS}_{2}, \mathrm{WSe}_{2}$, graphene, $\mathrm{SnS}, \mathrm{SnSe}, \mathrm{SnS}_{2}, \mathrm{SnSe}_{2}$ [86], InSe, $\mathrm{In}_{2} \mathrm{Se}_{3}, \mathrm{ReS}_{2}$, black AsP, $\mathrm{PtSe}_{2}, \mathrm{PtS}_{2}$, and $\mathrm{PdSe}_{2}$.

The $\mathrm{PdSe}_{2}$ layered material has remarkable optoelectronic properties, with a large bandgap tenability and extraordinary carrier mobility. The $\mathrm{PdSe}_{2}$-based devices are relatively stable and can be applied for photodetection from
Table 6 Detection bands of photodetectors and bandgaps depending on the types of $2 \mathrm{D}$ materials

\begin{tabular}{|c|c|c|c|c|c|}
\hline \multirow{2}{*}{$\begin{array}{l}\text { Material } \\
\mathrm{PdSe}_{2}\end{array}$} & \multirow{2}{*}{$\begin{array}{l}\text { Bandgap } \\
(\mathrm{eV}) \\
0-1.3\end{array}$} & \multirow{2}{*}{$\begin{array}{l}\text { References } \\
{[141]}\end{array}$} & \multicolumn{2}{|c|}{$\begin{array}{l}\text { Detection } \\
\text { bands }\end{array}$} & \multirow{2}{*}{$\begin{array}{l}\text { References } \\
{[19]}\end{array}$} \\
\hline & & & 532 & 1060 & \\
\hline & $0-1.43$ & [15] & n.a & 1060 & [41] \\
\hline \multirow[t]{2}{*}{$\mathrm{PtS}_{2}$} & $0.25-1.6$ & [142] & 500 & n.a & [143] \\
\hline & $0.25-1.6$ & [144] & 405 & 1550 & [144] \\
\hline \multirow[t]{2}{*}{$\mathrm{PtSe}_{2}$} & $0.3-1.2$ & [106] & 632 & 10,000 & [106] \\
\hline & $0-1.17$ & [142] & n.a & 980 & [11] \\
\hline \multirow[t]{2}{*}{$\mathrm{ReS}_{2}$} & 1.5 & [145] & 633 & n.a & [145] \\
\hline & 1.5 & [146] & 405 & 655 & [146] \\
\hline \multirow[t]{2}{*}{$\mathrm{ReSe}_{2}$} & 1.27 & [147] & 520 & n.a & [147] \\
\hline & $1.2-1.3$ & [148] & n.a & 808 & [148] \\
\hline \multirow[t]{2}{*}{ InSe } & 1.26 & [149] & 254 & 850 & [149] \\
\hline & 1.26 & {$[150]$} & 365 & 685 & [150] \\
\hline \multirow[t]{2}{*}{$\mathrm{In}_{2} \mathrm{Se}_{3}$} & 1.3 & {$[151]$} & 500 & 800 & [152] \\
\hline & 1.3 & [153] & 300 & 1100 & [153] \\
\hline \multirow[t]{2}{*}{ AsP } & $0.15-0.3$ & [105] & 2360 & 8050 & [105] \\
\hline & $0.1-0.3$ & [154] & 980 & n.a & [154] \\
\hline $\mathrm{SnS}$ & $1.0-1.2$ & [155] & 400 & 1050 & [155] \\
\hline $\mathrm{SnS}_{2}$ & 2.1 & [156] & 457 & 633 & [156] \\
\hline $\mathrm{SnSe}$ & $1.30-1.55$ & {$[155]$} & 400 & 1400 & [155] \\
\hline $\mathrm{SnSe}_{2}$ & $1-2$ & [86] & 300 & 2000 & [86] \\
\hline \multirow[t]{3}{*}{ Graphene } & 0 & [157] & n.a & 1550 & [157] \\
\hline & 0 & [158] & 285 & 1150 & [159] \\
\hline & 0 & [160] & 630 & 10,000 & [160] \\
\hline \multirow[t]{2}{*}{$\mathrm{WS}_{2}$} & $1.4-2.1$ & {$[161]$} & 365 & 650 & {$[161]$} \\
\hline & $1.4-2$ & [162] & 650 & 690 & [163] \\
\hline \multirow[t]{2}{*}{$\mathrm{WSe}_{2}$} & 1.63 & [164] & 500 & 900 & [164] \\
\hline & 1.2 & [165] & 473 & 1550 & [166] \\
\hline \multirow[t]{2}{*}{$\mathrm{MoS}_{2}$} & $1.35-1.82$ & [167] & 375 & 808 & [168] \\
\hline & 1.65 & [169] & 532 & 1070 & [169] \\
\hline \multirow[t]{2}{*}{$\mathrm{MoSe}_{2}$} & $8.4-1.1$ & [170] & 638 & n.a & [170] \\
\hline & 1.1 & {$[171]$} & n.a & 785 & [172] \\
\hline \multirow{2}{*}{$\begin{array}{l}\text { Phos- } \\
\text { phorene }\end{array}$} & 0.3 & [173] & 532 & 3390 & [174] \\
\hline & 0.3 & [175] & 400 & 3800 & [176] \\
\hline
\end{tabular}

deep ultraviolet to mid-infrared bands [21], and the longest photodetection wavelength studied thus far is $10.6 \mu \mathrm{m}$ [54].

\section{2 $\mathrm{PdSe}_{2}$ Photodetectors for Near-infrared Light Detection}

The near-infrared light $(1060 \mathrm{~nm})$ is important for optical data communication and biomedical imaging. The small 
bandgap of monolayer $\mathrm{PdSe}_{2}$ features resonant optical absorption of such a wavelength. Therefore, $\mathrm{PdSe}_{2}$ is an ideal material for near-infrared light photodetectors.

A typical $\mathrm{PdSe}_{2}$ photodetector has been measured under monochromatic illumination [19]. Because the $\mathrm{PdSe}_{2}$ photodetector is based on field-effect transistors, the gate voltage plays an important role in photodetection. The responsivity of the $\mathrm{PdSe}_{2}$ photodetector demonstrates a strong gate voltage dependence under 1.06- $\mu \mathrm{m}$ light illumination. The device showed an ultrahigh responsivity of $708 \mathrm{~A} \mathrm{~W}^{-1}$ at a gate voltage of $30 \mathrm{~V}$, and the detectivity was calculated to be $1.31 \times 10^{9}$ Jones.

The normal positive trend of the photocurrent increases with increasing power intensity [19]. The responsivity of the $\mathrm{PdSe}_{2}$ photodetector under $4.05-\mu \mathrm{m}$ illumination is much lower at $1.9 \mathrm{~mA} \mathrm{~W}^{-1}$. The photodetector exhibits excellent stability and repeatability in the environment at room temperature. The absorption spectra of $\mathrm{PdSe}_{2}$ flakes with different thicknesses demonstrate that the thick $\mathrm{PdSe}_{2}$ flakes have a higher MIR wavelength absorption. Therefore, this proves the feasibility of photodetection in the mid-infrared band.

However, the photoresponse time of $\mathrm{PdSe}_{2}$ photodetectors, in the order of several milliseconds, is less than desirable. The photogating effect may account for this phenomenon. That is, photogenerated electrons cannot recombine in a timely manner with photogenerated holes trapped by trap states. Therefore, the lifetime of photoelectrons is prolonged, and the device response is slow.

Both 2D materials and traditional 3D semiconductor materials can form heterostructures with $\mathrm{PdSe}_{2}$ and perform well in photodetection. A pyramid microstructure for heterojunction photodetectors have demonstrated their excellent performances via the light trapping effect and numerical modeling [62].

The $\mathrm{PdSe}_{2} /$ pyramid Si photodetector can achieve greater performance than that of the $\mathrm{PdSe}_{2} / \mathrm{Si}$ photodetector in terms of the responsivity, detectivity, and ON/OFF ratio [62], and they are compared with other heterostructures (discussed later in 6.3). The $\mathrm{PdSe}_{2}$ /pyramid Si photodetector can function as a self-driven device without a power supply. The tuning of the light intensity leads to a difference in the responsivity and ON/OFF ratio at zero bias. The maximum ON/OFF ratio can reach $1.6 \times 10^{5}$. The responsivity and detectivity depend on the illuminating light wavelength, and the maximum values are $456 \mathrm{~mA} \mathrm{~W}^{-1}$ and $9.97 \times 10^{13}$ Jones, respectively. Both are determined under 980-nm illumination for obtaining the peak sensitivity of the $\mathrm{PdSe}_{2} /$ pyramid Si photodetector.

Similar to the Si pyramid, Ge nanocones (GeNCs) in heterojunction photodetectors can absorb photons more efficiently [87]. They have a higher photocurrent than that of the $\mathrm{PdSe}_{2} /$ planar Ge heterostructure. Under 1550$\mathrm{nm}$ illumination with a power intensity of $5 \mu \mathrm{W} \mathrm{cm}$, the $\mathrm{PdSe}_{2} / \mathrm{GeNCs}$ photodetector exhibits a much larger responsivity $\left(530.2 \mathrm{~mA} \mathrm{~W}^{-1}\right.$ ) and quantum efficiency $(42.4 \%)$ than those under 1300-nm and 1650-nm illumination. The variation of the current ON/OFF ratios with light intensity was compared under three different wavelengths. This proves the best performance of the $\mathrm{PdSe}_{2} /$ GeNCs photodetectors in the 1550-nm detection.

\section{3 $\mathrm{PdSe}_{2}$ Photodetectors for Sensing Polarized Light}

Polarized light detection can be achieved in the heterostructures of $\mathrm{PdSe}_{2}$ with other materials, such as $\mathrm{PdSe}_{2} / \mathrm{Si}$ nanowire arrays (SiNWA) [25] and $\mathrm{PdSe}_{2} /$ perovskite [26] heterostructures.

Figure 19a demonstrates the schematic of the setup of the $\mathrm{PdSe}_{2} / \mathrm{SiNWA}_{\mathrm{N}}$ heterostructure-based photodetector. The responsivity $R$ and detectivity $D^{*}$ under various light intensities are shown in Fig. 19b. Both parameters increase with the decrease in light intensity and reach a maximum at $726 \mathrm{~mA} \mathrm{~W}^{-1}$ and $3.19 \times 10^{14}$ Jones upon illumination with a light intensity of $27.5 \mathrm{~cm}^{-2}$. Notably, the $\mathrm{PdSe}_{2} /$ SiNWA photodetector demonstrates a significant response to the weak light signals with a broad spectral detection range from the deep ultraviolet to the mid-infrared range (Fig. 19c).

However, it shows a high sensitivity to polarized light signals attributed to the asymmetric pentagonal structure of $\mathrm{PdSe}_{2}$. Here, the incident polarized light is supplied with various polarization angles through a half-wave plate using a polarizer. The normalized photocurrent was measured versus the polarization angle at zero bias (Fig. 19d). The polarization sensitivity of the $\mathrm{PdSe}_{2} / \mathrm{SiNWA}$ device is 75 , which is higher than that of other 2D material-based devices.

Therefore, the $\mathrm{PdSe}_{2} / \mathrm{SiNWA}_{\mathrm{N}}$ heterostructure exhibits great advantages as the self-driven and wide-band photodetector with highly polarization sensitivity. It has shown a remarkable broad photodetection from DUV to MIR with an excellent 
(a)

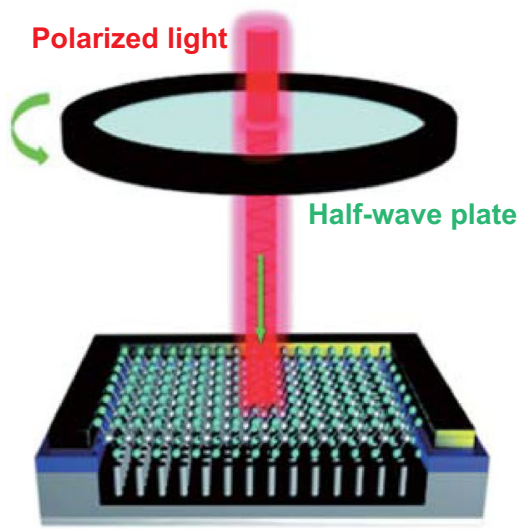

(c) 1

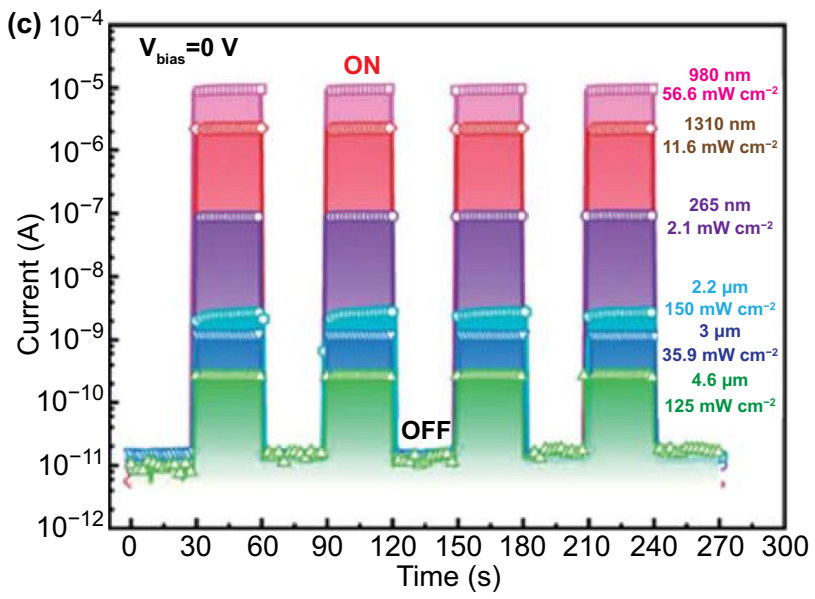

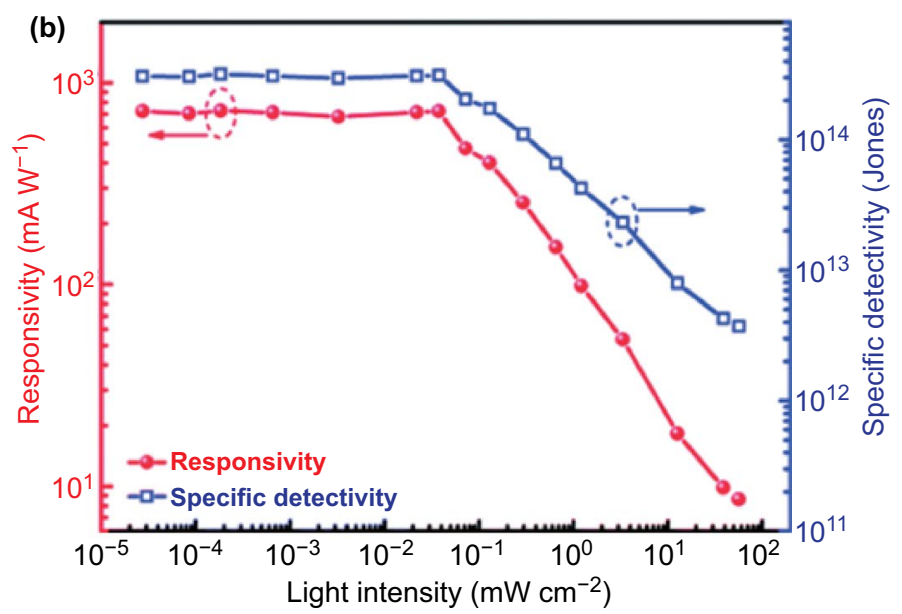

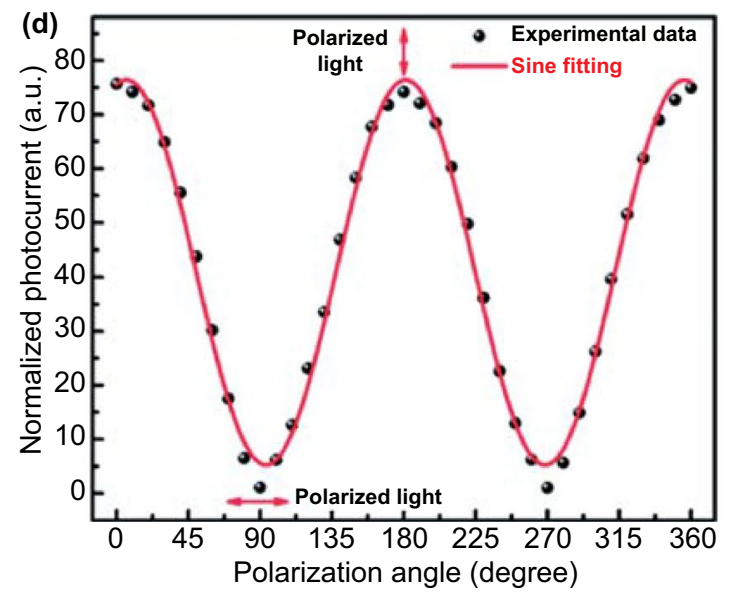

Fig. $19 \mathrm{PdSe}_{2}$ heterostructure-based photodetector for sensing polarized light. a Schematic illustration of photodetector based on PdSe ${ }_{2}$ and silicon nanowire arrays. b Light-intensity-dependent responsivity and detectivity of photodetector. $\mathbf{c}$ Time-dependent current of photodetector under illumination of infrared light with different wavelengths. Zero bias voltage applies. d Evolution of photocurrent under exposure of light with different polarization angles. The fitting curve approximates the sine function. Reprinted with permission from Ref. [25]. Copyright 2020, The Royal Society of Chemistry

responsivity, specific detectivity, response time, and polarization sensitivity. Meanwhile, the device holds prominent potential in infrared imaging of high pixel resolution.

Under $650-\mathrm{nm}$ illumination, the graphene/ $\mathrm{PdSe}_{2} / \mathrm{Ge}$ photodetector [21] shows a record polarization sensitivity (112.2) among the reported $\mathrm{PdSe}_{2}$-based devices, including $\mathrm{PdSe}_{2} / \mathrm{SiNWA}$ photodetectors (75) and $\mathrm{PdSe}_{2} /$ perovskite photodetectors (6.04) [26].

A comparison of polarized light sensing is presented for different 2D materials and their heterostructures (Table 7). The polarization sensitivity of the graphene/ $/ \mathrm{PdSe}_{2} / \mathrm{Ge}$ photodetector is much higher than that of some devices based on other 2D materials, such as $\mathrm{GeS}_{2}$ (2.1) [88], $\mathrm{GeSe}_{2}$ (2.16) [89], BP (8.7) [90], antimonene (17) [91], and $\mathrm{BP} / \mathrm{MoS}_{2}$ heterostructures (22) [92].

Analogous to silicene and black phosphorus, $\mathrm{PdSe}_{2}$ has a high sensitivity to polarized light owing to its anisotropic crystalline structure. Based on this, graphene/PdSe $/$ Ge heterojunction photodetectors have been studied for the polarization-dependent photoresponse [21].

Overall, $\mathrm{PdSe}_{2}$-based photodetectors demonstrate remarkable photodetection of broadband bands (from deep ultraviolet to mid-infrared), good responsivity, outstanding stability, and sensitive polarization. 
Table 7 Polarization sensitivity performance of 2D material-based photodetectors

\begin{tabular}{|c|c|c|c|}
\hline Material types & $\begin{array}{l}\text { Illumination } \\
\text { wavelength } \\
(\mathrm{nm})\end{array}$ & $\begin{array}{l}\text { Polarization } \\
\text { sensitivity }\end{array}$ & References \\
\hline Graphene/PdSe $2 / \mathrm{Ge}$ & 650 & 112.2 & {$[21]$} \\
\hline $\mathrm{PdSe}_{2} / \mathrm{Si}$ nanowire arrays & n.a & 75 & {$[25]$} \\
\hline Phosphorene/ $\mathrm{MoS}_{2}$ & 3500 & 22 & {$[92]$} \\
\hline Antimonene & 450 & 17 & [91] \\
\hline Phosphorene & 1550 & 8.7 & {$[90]$} \\
\hline $\mathrm{PdSe}_{2} /$ perovskite & 808 & 6.04 & {$[26]$} \\
\hline $\mathrm{GeSe}_{2}$ & n.a & 2.16 & {$[89]$} \\
\hline $\mathrm{GeS}_{2}$ & 325 & 2.1 & {$[88]$} \\
\hline GeSe & 532 & 1.3 & {$[177]$} \\
\hline $\mathrm{MoS}_{2} / \mathrm{GaAs}$ & 780 & 4.8 & [178] \\
\hline $\mathrm{ZnSb}$ & 1342 & 1.28 & [179] \\
\hline $\mathrm{PdSe}_{2}$ & 532 & 1.29 & [29] \\
\hline $\mathrm{PdS}_{2}$ & n.a & 0 & {$[180]$} \\
\hline $\mathrm{PtSe}_{2}$ & 10,000 & 0 & [106] \\
\hline $\mathrm{PtS}_{2}$ & 500 & 0 & [143] \\
\hline
\end{tabular}

\section{4 $\mathrm{PdSe}_{2}$ Photodetector-enhanced Humidity Sensors}

Besides image sensor, $\mathrm{PdSe}_{2}$-based devices can be applied to humidity sensors owing to the large surface-to-volume ratio of the $\mathrm{PdSe}_{2}$ film. For instance, a $\mathrm{PdSe}_{2} / \mathrm{SiNWA}_{\text {device }}$ has been utilized as a highly sensitive sensor of the relative humidity (RH) of the ambient environment [25].

Figure 20a shows the response performance of the $\mathrm{PdSe}_{2} /$ SiNWA devices at various relative humidity values from 11 to $95 \%$ in the dark. The response of the device exhibited good stability and repeatability at all $\mathrm{RH}$ values.

Moreover, the response of the $\mathrm{PdSe}_{2} / \mathrm{SiNWA}$ device under 780-nm illumination was significantly more sensitive than that in the dark (Fig. 20b). Figure 20c shows that the response speed is further improved under illumination when the $\mathrm{RH}$ value is $75 \%$, and the response and recovery times are superior to those of some sensors based on other materials reported previously. The rapid response of the device under illumination may have resulted from the rapid recombination of carriers. Figure 20d plots the incident light intensity dependence of the sensitivity at 75\% RH under 780-nm illumination, and the sensitivity of the device increases as the light intensity increases. Furthermore, the $\mathrm{PdSe}_{2} / \mathrm{SiNWA}$ device can retain its initial sensing performance after 6 months, indicating the good stability of the device [25].

\subsection{Saturable Absorber for Pulsed Laser}

Graphene has been employed as a saturable absorber in the formation of pulsed lasers in the visible to mid-infrared range. However, the zero bandgap of graphene hinders its photonic application. Moreover, TMDCs have been employed as $\mathrm{Q}$ switches or mode lockers in the generation of pulsed lasers such as $\mathrm{MoS}_{2}, \mathrm{WS}_{2}, \mathrm{MoSe}_{2}$, and $\mathrm{WSe}_{2}$. However, their bandgaps are tunable in a limited range, i.e., from 1 to $2 \mathrm{eV}$, which suppresses the potential for application in optical regulation. With a wide range of tunable bandgaps, phosphorene has shown remarkable performance as a saturable absorber in pulsed lasers [93]. However, its weak air stability impedes further studies.

The tunable bandgap and air stability have guaranteed that $\mathrm{PdSe}_{2}$ is a saturable absorber (SA) in passive Q-switching, which is a crucial method when fabricating pulsed laser devices [94].

A typical $\mathrm{PdSe}_{2}$-based passive Q-switched Nd:GdLaNbO laser is demonstrated (Fig. 21a). The laser diode (LD) as a direct pumping source is condensed into the $\mathrm{Nd}: \mathrm{GdLaNbO}_{4}$ crystal through the fiber core and a pair of convex lenses (L1, L2), and it is then transformed into a pulsed laser through the $\mathrm{PdSe}_{2}$ nanosheet, while the plane mirrors (M1, M2) are coated with the transmission of different reflectivity to control the output laser.

The pulse repetition frequency shows a positive correlation with the absorbed pump power (Fig. 21b), whereas the pulse duration displays a negative correlation. Figure 21c shows the evolution of the pulse energy and peak power of the $\mathrm{PdSe}_{2} / \mathrm{Nd}: \mathrm{GdLaNbO}_{4}$ laser with varying absorbed pump power, which may be due to the extensive modulation range of $\mathrm{PdSe}_{2}$. These results are better than those of $\mathrm{MoS}_{2}$ and $\mathrm{WS}_{2}$ [95], proving the excellent characteristics of the $\mathrm{PdSe}_{2} \mathrm{SA}$ and the excellent potential of passive Q-switched lasers.

Due to the suitable bandgaps, 2D materials have been employed as saturable absorbers (SA) for passively Q-switched and mode-locked fiber laser. Besides, optical circuits have incorporated various saturable absorber materials, such as SnTe quantum dots, graphitic-phase $\mathrm{C}_{3} \mathrm{~N}_{4}, \mathrm{MoS}_{2}, \mathrm{PdS}_{2}$, $\mathrm{In}_{2} \mathrm{Se}_{3}, \mathrm{PtS}_{2}, \mathrm{WS}_{2}$, and $\mathrm{PdSe}_{2}$ [96]. Indeed, they have emerged 

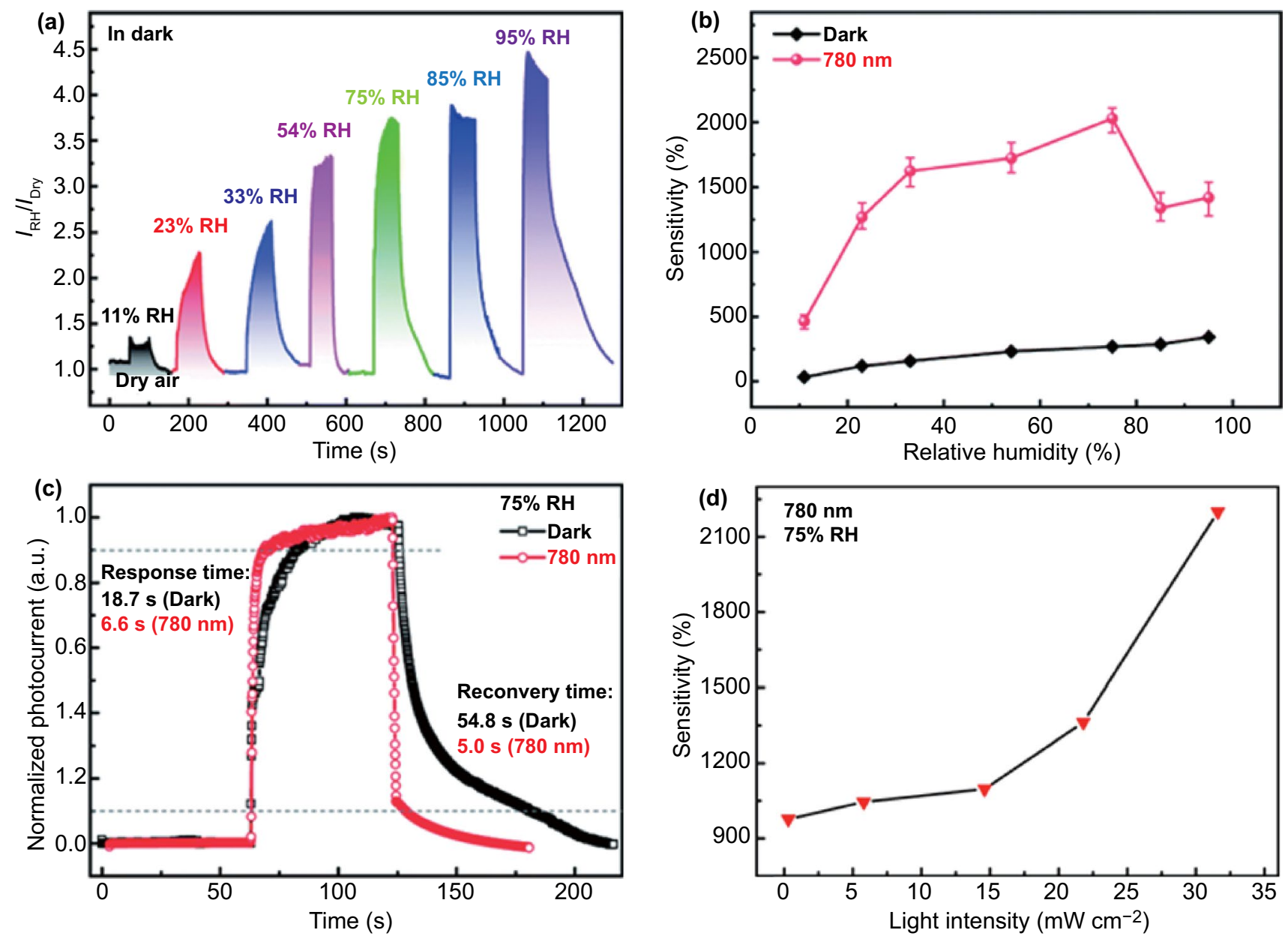

Fig. 20 a Variation of current ratio of $\mathrm{PdSe}_{2}$-based device with relative humidity in the dark. b Relative humidity dependence of sensitivity in the dark and under 780-nm illumination. c Temporal response of $\mathrm{PdSe}_{2}$-based device at $75 \% \mathrm{RH}$ in the dark and under 780-nm illumination. d Light intensity dependence of sensitivity at $75 \% \mathrm{RH}$ under $780 \mathrm{~nm}$. The RH denotes the relative humidity. Reprinted with permission from Ref. [25]. Copyright 2020, Royal Society of Chemistry

as cost-effective, simple, and highly integrated component for pulsed laser generation.

Future works may lie in the adoption of $\mathrm{PdSe}_{2}$-based van der Waals heterostructures as saturable absorbers for pulsed laser modulation in the fiber lasers or solid-state lasers.

Previously, the electronics, optoelectronics, and optics of $\mathrm{PdSe}_{2}$ have been introduced. Besides, the $\mathrm{PdSe}_{2}$ may possess great promises in the environmental, energy and biomedical applications. Indeed, the 2D materials have demonstrated the great performances in clean energy production [97-99], i.e., catalysis of hydrogen production or oxygen reduction, solar cells [100], thermoelectric power generation, energy storage, environmental remediation [101, 102], and photodegradation of organic-molecules-polluted water [103] as well as water purification. Besides, the metallic low dimension materials may favor the anti-bacterial performances as well as other biomedical engineering.

After knowing the devices of individual $\mathrm{PdSe}_{2}$ material, we come to the discussion of $\mathrm{PdSe}_{2}$-based van der Waals heterostructures.

\section{$6 \mathrm{PdSe}_{2}$-based van der Waals Heterostructures}

The vdWHs of 2D materials employ weak layer interactions between two stacked layered materials to form multilayer structures. Owing to the enriched choice 
(a)
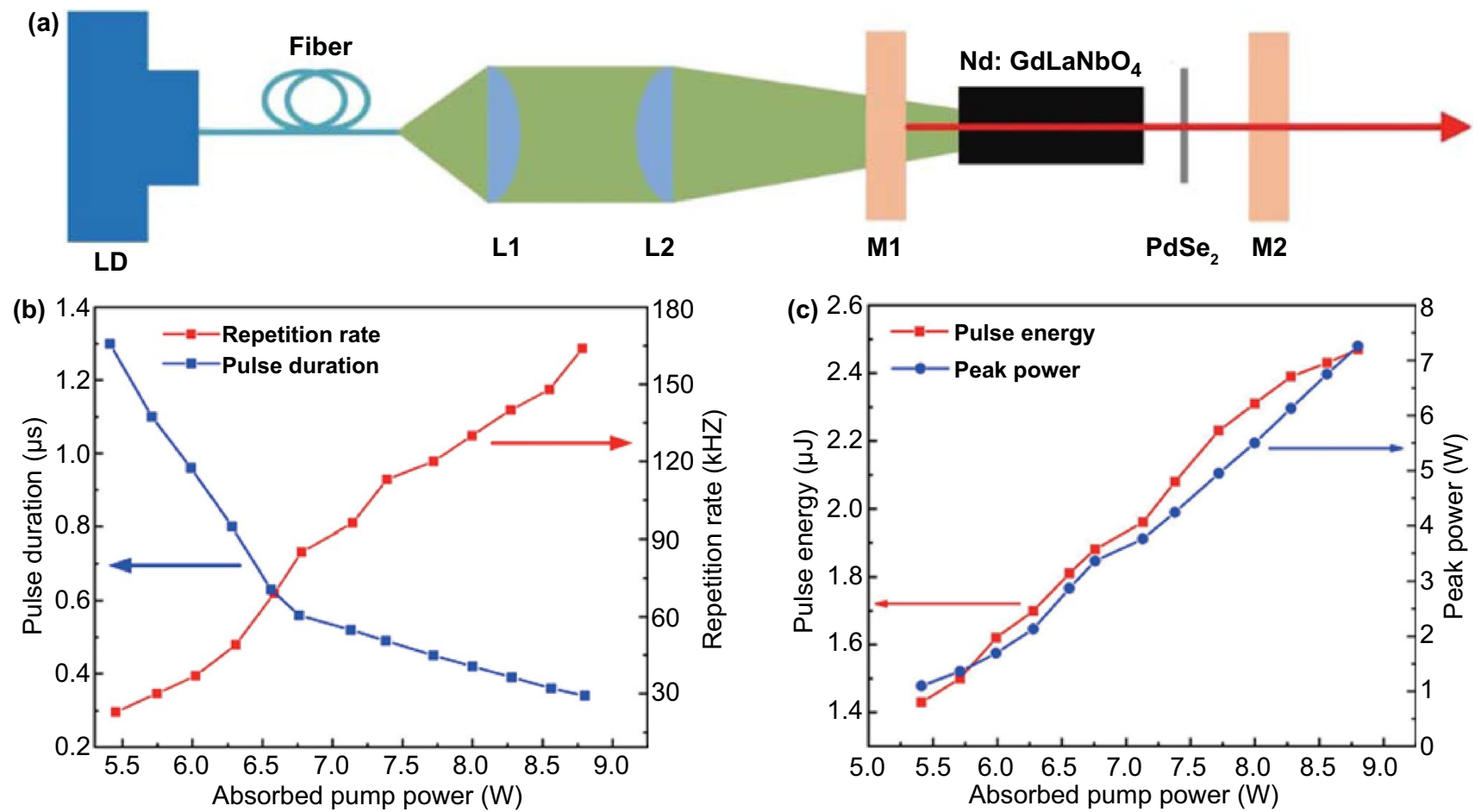

Fig. 21 a Schematic illustration of $\mathrm{PdSe}_{2}$ passively Q-switched $\mathrm{Nd}$ :GdLaNbO ${ }_{4}$ pulsed laser experimental setup. b Pulse duration (left) and repetition rate (right) versus absorbed pump power. c Variation of the pulse energy (left) and pulse peak power (right) with the absorbed pump power. Reprinted with permission from Ref. [94]. Copyright 2020, Elsevier Ltd

of conductivity types, 2D materials can be stacked by choosing from semiconducting, metallic, and insulating types. Indeed, 2D material-based vdWHs have enhanced the device architectures of conventional $\mathrm{Si}$ technology. Here, $\mathrm{PdSe}_{2}$ as a semiconducting 2D material could broaden the applicability of $2 \mathrm{D}$ vdWHs. In this section, we discuss emerging applications in electronics, such as rectifiers and optoelectronics, such as image sensors.

\subsection{Van der Waals Heterostructure Based on $\mathrm{PdSe}_{2}$ / $\mathrm{MoS}_{2}$ Contact}

Two-dimensional heterojunction-based photodetectors show superior photoresponse time and detectivity. $\mathrm{PdSe}_{2} / \mathrm{MoS}_{2}$ vdWH photodetectors (Fig. 22a) can effectively improve the responsivity and detectivity under 10.6- $\mu \mathrm{m}$ illumination, and the rise/fall time $\left(\tau_{\mathrm{r}} / \tau_{\mathrm{f}}\right)$ of the photocurrent is $65.3 / 62.4 \mu \mathrm{s}$ [54].

The vdWH can significantly suppress the dark current and current noise of the device, and the photocurrent can be generated under the combined action of intralayer excitons and interlayer excitons [54].

Figure $22 \mathrm{~b}$ shows the responsivity and noise equivalent power as a function of the incident wavelength. $\mathrm{PdSe}_{2}$-based heterojunction photodetectors have better responsivity and detectivity for broadband detection. Typical photocurrent performances are presented in the dark and under illumination (Fig. 22c).

The photoresponse time can be improved by fast charge transfer in the heterostructure. Indeed, the $\mathrm{PdSe}_{2} /$ perovskite heterojunction photodetector could solve the problems faced by most perovskite photodetectors, i.e., low specific detectivity and slow photoresponse [104].

The detectivity of $\mathrm{PdSe}_{2} / \mathrm{MoS}_{2}$ photodetectors can reach $8.21 \times 10^{9}$ Jones, which is much better than that of most mid-infrared photodetectors (Fig. 22d) based on AsP [105], $\mathrm{PtSe}_{2}$ [106], graphene thermopiles [107], and uncooled $\mathrm{HgCdTe}$ [108]. The detectivity of $\mathrm{PdSe}_{2}$ exceeded that of some traditional mid-infrared photodetectors [108]. Compared with the $\mathrm{PdSe}_{2} / \mathrm{MoS}_{2}$ 

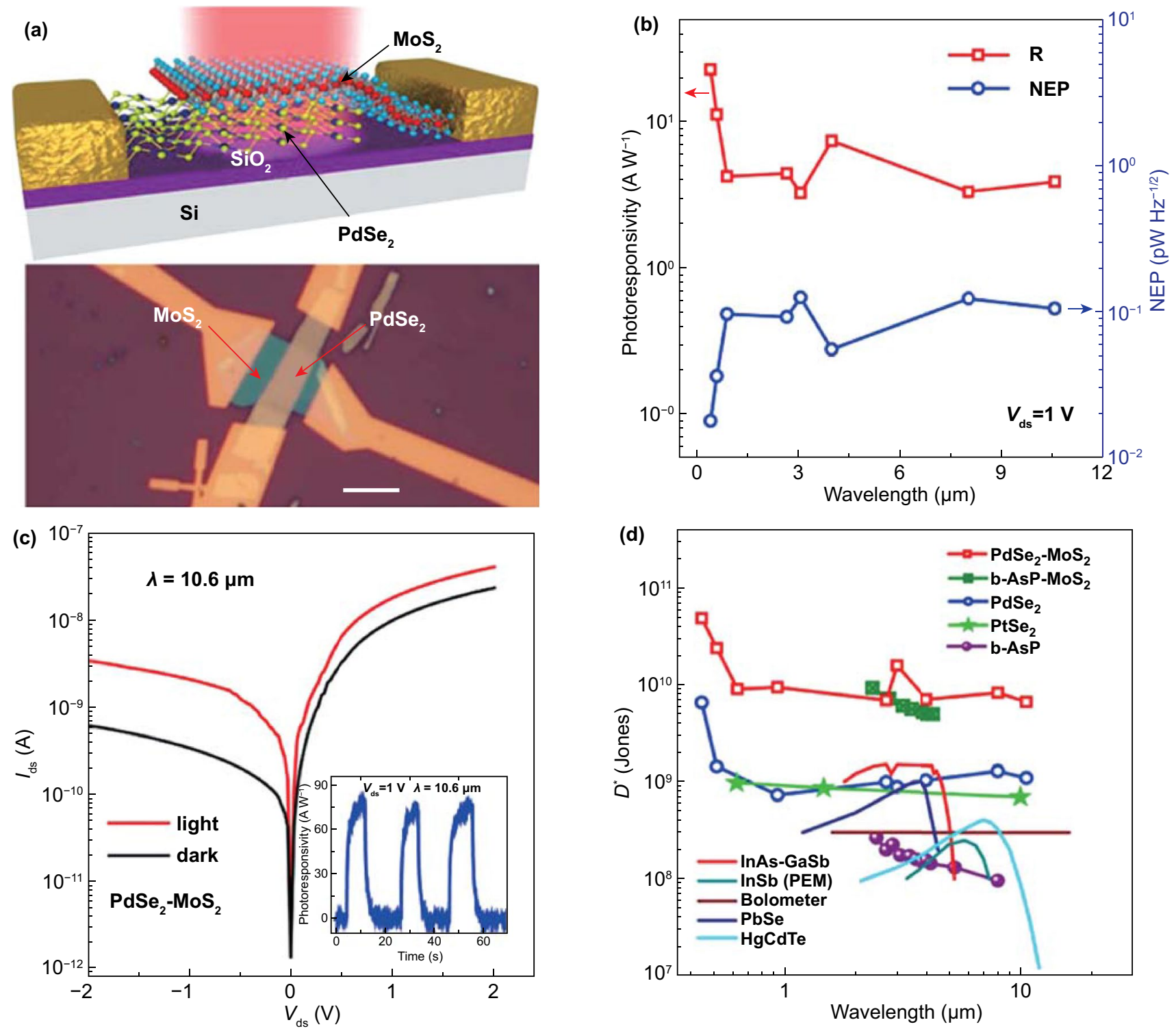

Fig. $22 \mathrm{PdSe}_{2} / \mathrm{MoS}_{2}$ van der Waals heterostructure-based photodetector. a Scheme of the $\mathrm{PdSe}_{2} / \mathrm{MoS}_{2}$ photodetector (top) and optical micrograph of the corresponding device (bottom), where the scale bar is $5 \mu \mathrm{m}$. b Wavelength dependence of photoresponsivity (red) and noise equivalent power (blue) of the $\mathrm{PdSe}_{2} / \mathrm{MoS}_{2}$ photodetector at $\mathrm{V}_{\mathrm{DS}}=1 \mathrm{~V}$. NEP denotes noise equivalent power. $\mathrm{c}$ Drain current of heterostructure-based device under illumination and in the dark. The inset shows the current profile against time with periodic light illumination and dark state. d Wavelength-dependent detectivity of different 2D materials and some infrared materials at room temperature. Reprinted with permission from Ref. [54]. Copyright 2019, American Chemistry Society

photodetector (Table 8), the potential of $\mathrm{PdSe}_{2}$ in midinfrared photodetection is further reflected.

\section{2 $\mathrm{PdSe}_{2}$ van der Waals Heterostructure-based $p-n$ Junction-based Rectifier}

The optoelectronics has stemmed from the fundamental component of $p-n$ junctions. Indeed, the conventional 3D thin film stacking has contributed to the photovoltaics $[109,110]$, photodetectors, tunneling transistors, rectifiers, and light-emitting diodes. The metal/semiconductor contact has favored the Ohmic type conductance behavior for elevating the charge carrier transport. These investigations based on thin film deposition techniques have provided useful guide for 2D materials. 
Table 8 Detectivity performance of 2D materials in mid-infrared photodetectors

\begin{tabular}{llll}
\hline Materials & $\begin{array}{l}\text { Illumination } \\
\text { wavelength } \\
(\mathrm{nm})\end{array}$ & Detectivity (Jones) & References \\
\hline $\mathrm{PdSe}_{2} / \mathrm{MoS}_{2}$ & 10,600 & $8.21 \times 10^{9}$ & {$[54]$} \\
$\mathrm{AsP}^{\mathrm{P} P \mathrm{Se}_{2}}$ & 5000 & $4.9 \times 10^{9}$ & {$[105]$} \\
$\begin{array}{l}\text { graphene thermo- } \\
\text { piles }\end{array}$ & 10,000 & $7 \times 10^{8}$ & {$[106]$} \\
$\begin{array}{l}\text { uncooled } \mathrm{HgCdTe} \\
\text { n.a }\end{array}$ & 9000 & $8 \times 10^{8}$ & {$[107]$} \\
\hline
\end{tabular}

Two types of 2D materials stack together with weak interaction, termed van der Waals heterostructure. With delicate selection, one can assembly a $p-n$ junction with the atomic layer thickness $[111,112]$. No dangling bonds remain at their interface; besides, low lattice mismatch between both 2D materials result in the declined defect states. Therefore, the quantity of scattering center for charge carrier is minimized for boosting the charge carrier transport, which is superior to the Si based materials.
High gate-modulated rectification in vdWHs based on $\mathrm{PdSe}_{2}$ has been introduced and examined. For example, $p$-type germanium selenide $(\mathrm{GeSe})$ and $n$-type $\mathrm{PdSe}_{2}$ with a pure ohmic contact show a large rectification ratio, which is defined as the ratio between the forward and reverse currents, up to $5.5 \times 10^{5}$, resulting from the clean interface and low Schottky barrier [24].

One can find schematic of the $p-\mathrm{GeSe} / n-\mathrm{PdSe}_{2} \mathrm{vdWH}-$ based rectifier device (Fig. 23a), and the corresponding optical image (Fig. 23b).

Figure 23c displays the AFM images of the GeSe and $\mathrm{PdSe}_{2}$ flakes with thicknesses of 12 and $11.5 \mathrm{~nm}$, respectively. The Raman spectra of $\mathrm{GeSe}$ and $\mathrm{PdSe}_{2}$ demonstrate the successful stacking of both 2D materials (Fig. 23d).

For $p-\mathrm{GeSe} / n-\mathrm{PdSe}{ }_{2}$ diodes, the linear scale (Fig. 23e) and the semi-log scale (Fig. 23f) of the drain current versus voltage curves were measured at different gate voltages. Indeed, the gate voltage can modulate the rectifying effect. This result is due to the carrier density and electrostatic inversion from semiconductor to semi-insulator materials [113]. Figure $23 \mathrm{~g}$ presents the variation in the ideality factor $\eta$ of the $p-n$ diodes, which is obtained as 1.2 at a negative gate voltage. The $p$ - $n$ diode tends to decrease its ideality at (a)

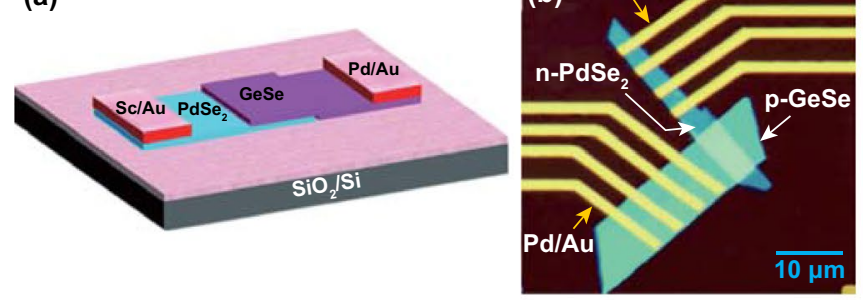

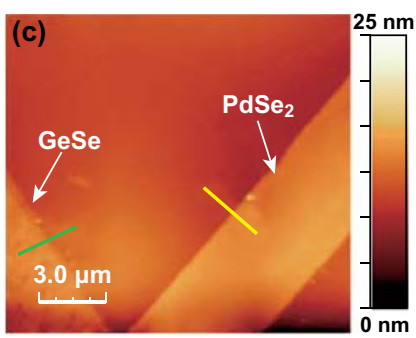

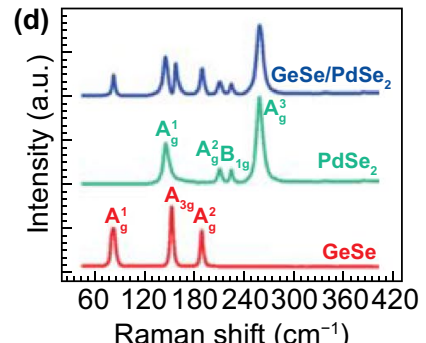

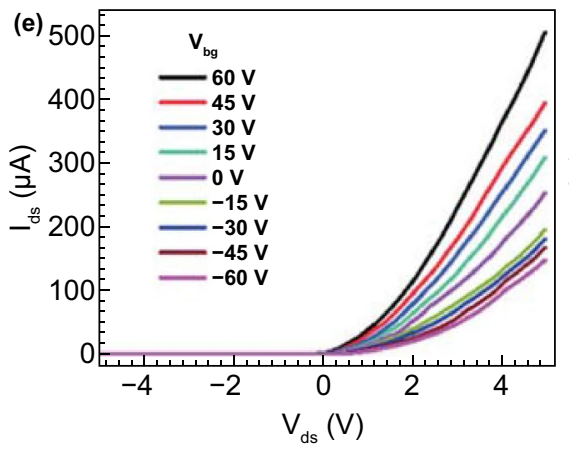
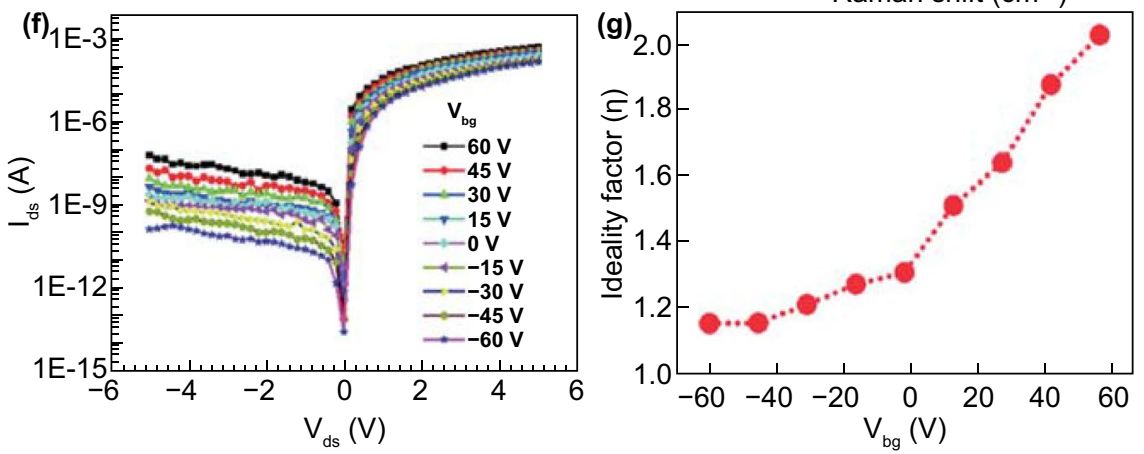

Fig. $23 \mathrm{GeSe} / \mathrm{PdSe}_{2}$ junction-based rectifier. a Schematic of the GeSe/PdSe ${ }_{2} p-n$ junction. b Optical micrograph of the GeSe/PdSe ${ }_{2}$ junction with $\mathrm{Pd} / \mathrm{Au}$ and $\mathrm{Sc} / \mathrm{Au}$ electrodes. c AFM image of the GeSe/PdSe ${ }_{2}$ heterostructure. d Raman spectra of the GeSe/PdSe $\mathrm{H}_{2}$ junction and individual flakes. e, $\mathbf{f}$ Drain current versus voltage curves of the device in a linear scale and semi-log scale with sweeping different back gate voltages. $\mathbf{g}$ Gate voltage dependence of the ideality factor of the device. Reprinted with permission from Ref. [24]. Copyright 2020, The Royal Society of Chemistry 
a positive gate voltage, which can be attributed to the carrier recombination at the sharp interface resulting from the decrease in electric field [114]. Table 9 compares the rectification ratios of typical $p$ - $n$ diodes based on the vdWHs of $\mathrm{PdSe}_{2}$ and other 2D materials.

This proves that nTMDC-based rectifier may hold promises in logic switches as shown in other TMDC logic circuits [115]. Besides, the nTMDC-based rectifier could be employed as an energy harvester for collecting the electromagnetic wave energy as proved by other $2 \mathrm{D}$ materials [116].

\section{3 $\mathrm{PdSe}_{2}$ van der Waals Heterostructure-based Junction Photodetectors}

The PdSe $\mathrm{P}_{2}$ based van der Waals heterostructures remain less investigated in terms of fabrication strategies. One can refer to the investigation of other vdWH emerging 2D materials. To date, the dry stamp transfer method has dominated the stacking nanosheets. Indeed, the epitaxy-based synthesis has great opportunities of fabricating the secondary layer of 2D materials. Besides, large quantity of 2D materials remain unexplored for the stacking of 2D materials such as metal-organic framework, graphene, $\mathrm{MoS}_{2}, \mathrm{ReSe}_{2}, \mathrm{PtSe}_{2}$, MXene, and tellurium as well as perovskites. Besides, the lateral heterostructure may arise the attention for novel charge carrier transport.

To investigate the additional features of the $p-n \mathrm{vdWH}$ diode, the photoresponse was investigated [117]. Figure 24a shows a schematic of the $p-\mathrm{BP} / n-\mathrm{PdSe}_{2}$ vdWH diode under illumination.

The time-resolved photocurrent was measured under intermittent lasers with different wavelengths at a fixed

Table 9 Rectification ratio of $p-n$ junction-based diodes with different van der Waals heterostructures based on 2D materials

\begin{tabular}{|c|c|c|}
\hline$p-n$ junction diodes & Rectification ratio & References \\
\hline $\mathrm{GeSe} / \mathrm{PdSe}_{2}$ & $5.5 \times 10^{5}$ & [24] \\
\hline Phosphorene/PdSe ${ }_{2}$ & $7.1 \times 10^{5}$ & [117] \\
\hline Phosphorene/MoS 2 & $1 \times 10^{5}$ & [181] \\
\hline $\mathrm{GaSe} / \mathrm{InSe}$ & $1 \times 10^{5}$ & [182] \\
\hline Graphene/WSe ${ }_{2}$ & $1 \times 10^{4}$ & [115] \\
\hline $\mathrm{WSe}_{2} / \mathrm{MoS}_{2}$ & $1 \times 10^{4}$ & [183] \\
\hline $\mathrm{WSe}_{2} / \mathrm{SnSe}_{2}$ & $2.1 \times 10^{4}$ & {$[184]$} \\
\hline $\mathrm{MoS}_{2} / \mathrm{WSe}_{2}$ & $1.3 \times 10^{5}$ & {$[185]$} \\
\hline $\mathrm{WSe}_{2} / \mathrm{GeSe}$ & $1 \times 10^{5}$ & [186] \\
\hline
\end{tabular}

power (Fig. 24b). The varying incident wavelengths from the visible to NIR region on the $p-\mathrm{BP} / n-\mathrm{PdSe}_{2}$ diode led to current versus voltage curves (Fig. 24c). This indicates that the photocurrent decreased when the incident wavelength increased. The photocurrent of the diode depends on the back-gate voltage (Fig. 24d).

The energy band alignment of $p$-BP, $n$-PdSe $\mathrm{Pe}_{2}$, and their heterostructures after contact (Fig. 24e), with the CBM, VBM, work function, and electron affinity. A magnified view of the band alignment is presented after contact at a gate voltage of $0 \mathrm{~V}$ (Fig. 24e). When the diode operates at a negative back-gate voltage, the Fermi level moves away from the conductance band. This increases the potential barrier of the $p-\mathrm{BP} / n-\mathrm{PdSe}_{2}$ interface, resulting in a high rectifying current. The Fermi level approaches the conductance band at a negative gate voltage (Fig. 24f) and decreases the potential barrier and rectification ratio. For the $p-\mathrm{BP} / n-\mathrm{PdSe}_{2}$ diode, the positive gate voltage (Fig. 24g) can modulate the Fermi level and control the carrier densities, which can eventually control the rectification ratio.

Therefore, the $\mathrm{PdSe}_{2} p-n$ junction-based photodiode shows a great potential in high-performance visible-infrared photodetectors, as well as solar cell for electricity production. This $p$ - $n$ diode concept may broaden the application of 2D nTMDC-based heterostructures in photovoltaics.

In this section, we discuss the structure and performance of different photodetectors based on $\mathrm{PdSe}_{2}$. A comparison of the performances of different $\mathrm{PdSe}_{2}$-based photodetectors is listed in Table 10.

\subsection{Image Sensor System from $\mathrm{PdSe}_{2}$ van der Waals Heterostructure}

Because of the excellent performance of $\mathrm{PdSe}_{2}$ in the field of photodetection, some studies subsequently explored further possibilities in the image sensor field. Infrared image sensors have emerged as an essential device unit in optoelectronic systems such as fire monitoring, night vision, and surveillance cameras [118].

The $\mathrm{PdSe}_{2} /$ pyramid Si device presented superior results in terms of infrared image sensing [119]. In portable systems, cardboard masks can be imaged using such a device. The geometry of the house and tree shapes was imaged under 980-nm and 1300-nm illumination. The illuminated areas are highlighted in photocurrent mapping. In contrast, the 

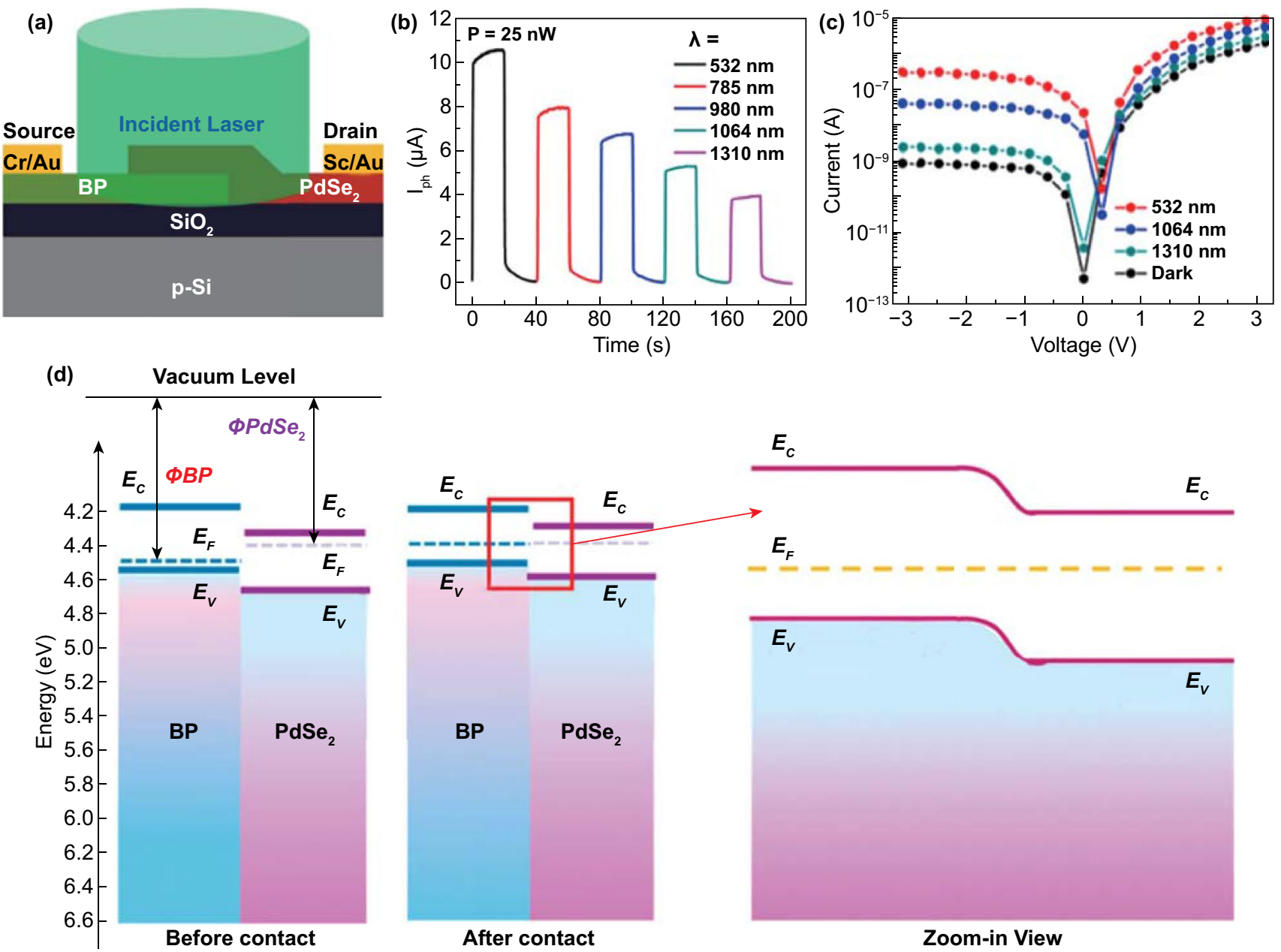

(e)

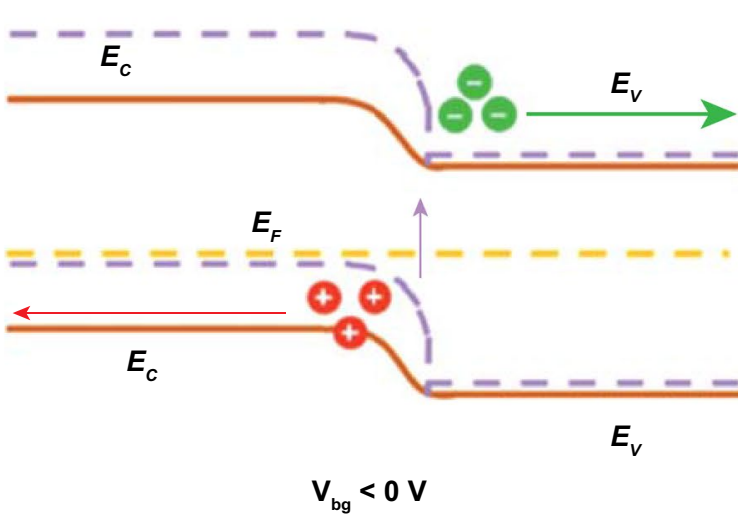

(f)

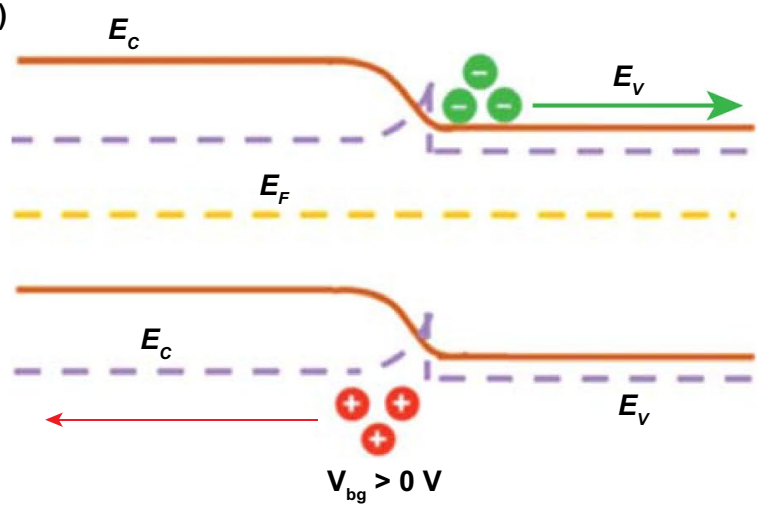

Fig. 24 a Schematic of the $p-\mathrm{BP} / n-\mathrm{PdSe}_{2} p-n$ diode under illumination. b Time-dependent $\mathrm{I}_{\mathrm{ph}}$ of the $p$ - $\mathrm{BP} / n$ - $\mathrm{PdSe}{ }_{2}$ photodiodes under the illumination of different wavelengths. $\mathbf{c} \mathrm{I}_{\mathrm{DS}}-\mathrm{V}_{\mathrm{DS}}$ curves under the illumination of different wavelengths at back gate voltage of $10 \mathrm{~V}$. d Energy band of the $p-\mathrm{BP} / n-\mathrm{PdSe}_{2}$ van der Waals heterojunction before contact and after contact with a zoom-in view. Energy band alignment of a $p$-BP $/ n-\mathrm{PdSe} e_{2}$ photodiode under $\mathbf{e}$ a negative back-gate voltage and $\mathbf{f}$ a positive back-gate voltage. Reprinted with permission from Ref. [117]. Copyright 2020, American Chemical Society 
Table 10 Performances of photodetectors based on 2D PdSe ${ }_{2}$ and its van der Waals heterostructures as well as related nanostructures

\begin{tabular}{|c|c|c|c|c|c|c|}
\hline Materials & $\begin{array}{l}\text { Wavelength or } \\
\text { band } \lambda(\mathrm{nm})\end{array}$ & $\begin{array}{l}\text { Responsivity } R \\
\left(\mathrm{~mA} \mathrm{~W}^{-1}\right)\end{array}$ & Detectivity $D^{*}$ (Jones) & $\mathrm{I}_{\text {light }} / \mathrm{I}_{\text {dark }}$ & $\tau_{\mathrm{r}} / \tau_{\mathrm{f}}$ & $\begin{array}{l}\text { Refer- } \\
\text { ences }\end{array}$ \\
\hline $\mathrm{PdSe}_{2} / \mathrm{MoS}_{2}$ & 10,600 & $4.21 \times 10^{4}$ & $8.2 \times 10^{9}$ & 10 & $65.3 / 62.4 \mu \mathrm{s}$ & {$[54]$} \\
\hline $\mathrm{PdSe}_{2} / \mathrm{GeNCs}$ & 1550 & 530.2 & $1.45 \times 10^{11}$ & $7 \times 10^{3}$ & $25.4 / 38.5 \mu \mathrm{s}$ & [87] \\
\hline $\mathrm{PdSe}_{2}$ & 1060 & $7.08 \times 10^{5}$ & $1.31 \times 10^{9}$ & 10 & $220 \mathrm{~ms}$ & [19] \\
\hline $\mathrm{PdSe}_{2}$ & 1060 & n.a & n.a & n.a & $156 / 163 \mu \mathrm{s}$ & [41] \\
\hline $\mathrm{PdSe}_{2} / \mathrm{Ge}$ & 980 & 691.5 & $1.73 \times 10^{13}$ & $10^{5}$ & $6.4 / 92.5 \mu \mathrm{s}$ & {$[21]$} \\
\hline $\mathrm{PdSe}_{2} /$ pyramid Si & 980 & 456 & $9.97 \times 10^{13}$ & $1.6 \times 10^{5}$ & n.a & [119] \\
\hline $\mathrm{PdSe}_{2} / \mathrm{SiNWA}$ & 980 & 726 & $3.19 \times 10^{14}$ & $10^{6}$ & $3.4 / 3.9 \mu \mathrm{s}$ & {$[25]$} \\
\hline $\mathrm{PdSe}_{2} /$ perovskite & 808 & 313 & $2.72 \times 10^{13}$ & $10^{4}$ & $3.5 / 4 \mu \mathrm{s}$ & [26] \\
\hline $\mathrm{PdSe}_{2} / \mathrm{Si}$ & 780 & 300.2 & $1.18 \times 10^{13}$ & $1.08 \times 10^{5}$ & $38 / 44 \mu \mathrm{s}$ & {$[62]$} \\
\hline $\mathrm{PtSe}_{2} / \mathrm{SiNWA}$ & $200-1550$ & $1.265 \times 10^{4}$ & $2.5 \times 10^{13}$ & $4 \times 10^{4}$ & $10.1 / 19.5 \mu \mathrm{s}$ & [7] \\
\hline $\mathrm{PtSe}_{2} / n-\mathrm{GaN}$ & 265 & 193 & $3.8 \times 10^{14}$ & $10^{8}$ & $45 / 102 \mu \mathrm{s}$ & {$[82]$} \\
\hline $\mathrm{PtS}_{2}$ & 500 & $1.56 \times 10^{6}$ & $2.9 \times 10^{11}$ & n.a & $0.46 \mathrm{~s}$ & [143] \\
\hline $\mathrm{WS}_{2} / p-\mathrm{Si}$ & $340-1100$ & $5.7 \times 10^{3}$ & n.a & 10.65 & $670 / 998 \mu \mathrm{s}$ & [187] \\
\hline $\mathrm{WS}_{2}$ & 365 & $5.35 \times 10^{4}$ & $1.22 \times 10^{11}$ & n.a & n.a & {$[161]$} \\
\hline $\mathrm{NiPS}_{3}$ & 254 & 126 & $1.22 \times 10^{12}$ & 200 & $3.2 / 15.6 \mathrm{~ms}$ & [188] \\
\hline $\mathrm{BP}$ & $640-940$ & 4.8 & $\mathrm{n} . \mathrm{a}$ & n.a & $1 / 4 \mathrm{~ms}$ & [83] \\
\hline $\mathrm{MoS}_{2} / \mathrm{BP}$ & $532-1550$ & 153 & n.a & n.a & $15 / 70 \mu \mathrm{s}$ & [189] \\
\hline $\mathrm{MoS}_{2} / n-\mathrm{Si}$ & $300-1100$ & $1.19 \times 10^{4}$ & $2.1 \times 10^{10}$ & 59.9 & $30.5 / 71.6 \mu \mathrm{s}$ & {$[190]$} \\
\hline $\mathrm{MoS}_{2} /$ Graphene & $420-980$ & 835 & n.a & n.a & $20 / 30 \mathrm{~ms}$ & [191] \\
\hline $\mathrm{MoS}_{2} / p-\mathrm{GaN}$ & 265 & 187 & $2.34 \times 10^{13}$ & $10^{5}$ & $46.4 / 114.1 \mu \mathrm{s}$ & {$[85]$} \\
\hline $\mathrm{GaN}$ & 325 & 340 & $1.24 \times 10^{9}$ & n.a & $280 / 450 \mathrm{~ms}$ & [192] \\
\hline $\mathrm{GaSe} / \mathrm{GaSb}$ & $400-1800$ & 115 & $1.3 \times 10^{12}$ & n.a & $32 / 24 \mu \mathrm{s}$ & [193] \\
\hline $\mathrm{InGaAs} / p-\mathrm{Si}$ & $400-1250$ & $7.52 \times 10^{3}$ & n.a & n.a & $13 / 16 \mathrm{~ms}$ & [194] \\
\hline $\mathrm{MgO}$ & 150 & $1.86 \times 10^{3}$ & $1.8 \times 10^{10}$ & $10^{2}$ & $\mathrm{n} . \mathrm{a}$ & [195] \\
\hline Graphene/Ge & $1200-1600$ & 51.8 & $1.38 \times 10^{10}$ & $2 \times 10^{4}$ & $23 / 108 \mu \mathrm{s}$ & [196] \\
\hline Graphene $/ n-\mathrm{Si}$ & $300-1100$ & 730 & $4.2 \times 10^{12}$ & $10^{4}$ & $320 / 750 \mu \mathrm{s}$ & [197] \\
\hline Graphene/MoTe $2 /$ Graphene & 1064 & 110 & $10^{10}$ & n.a & $24 / 46 \mu \mathrm{s}$ & [198] \\
\hline Graphene/Si & 365 & 120 & $6.1 \times 10^{12}$ & $10^{5}$ & 4/12 ns & [84] \\
\hline
\end{tabular}

photocurrent in the other areas remained much weaker, similar to the dark state.

Although some blemishes in the blocked regions need to be further corrected, the shapes of the patterns can be distinguished easily by contrast. Similar results were obtained for the $\mathrm{PdSe}_{2} / \mathrm{GeNCs}$ hybrid device [263], indicating the reliable infrared imaging capability of $\mathrm{PdSe}_{2}$-based devices.

The suitable bandgap of $\mathrm{PdSe}_{2}$ guarantees its application in infrared light sensing. When the devices are fabricated into arrays, the system can achieve image sensing with high pixel numbers [21]. When the polarized light is incident on the device through a specific mask, the lock-in amplifier can timely scan the voltage of the device and transform it into the voltage mapping image.
The graphene $/ \mathrm{PdSe}_{2} / \mathrm{Ge}$ photodetector with a broadband range from ultraviolet to near-infrared light (Fig. 25a).

The normalized photocurrent of the graphene/ $\mathrm{PdSe}_{2} / \mathrm{Ge}$ device shows a strong correlation with the polarization angle under various illuminations having different wavelengths, including 365, 650, 980, and $1550 \mathrm{~nm}$ (Fig. 25b).

The maximum and minimum values of the photocurrent were achieved at polarization angles of $0^{\circ}\left(180^{\circ}\right)$ and $90^{\circ}$ $\left(270^{\circ}\right)$, respectively. Indeed, the sine-shaped photocurrent curve indicates a good polarization sensitivity of the graphene/ $\mathrm{PdSe}_{2} / \mathrm{Ge}$ device [21].

A high-resolution infrared image is compiled after projecting a patterned light to the detector (Fig. 25c) after passing through the $P$ letter mask (Fig. 25d). Here, the photocurrent intensity is stronger with a polarization 
(a)

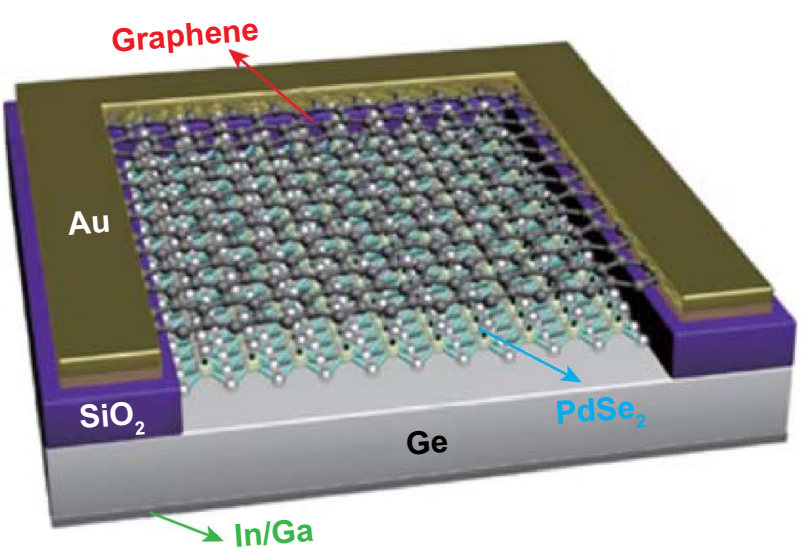

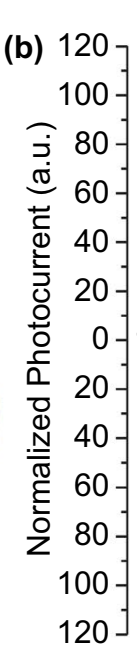

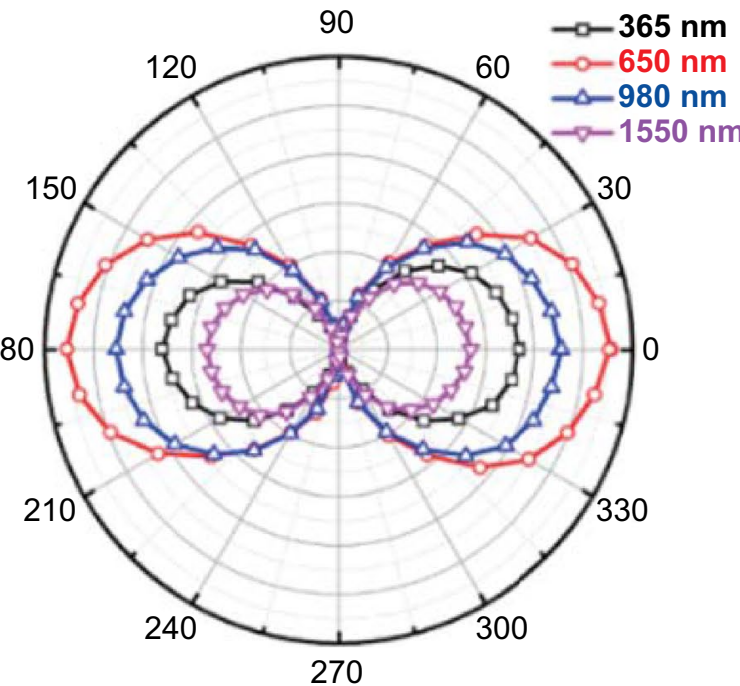

(c)

Polarizer

Mask

Chopper

Photodetector

Light

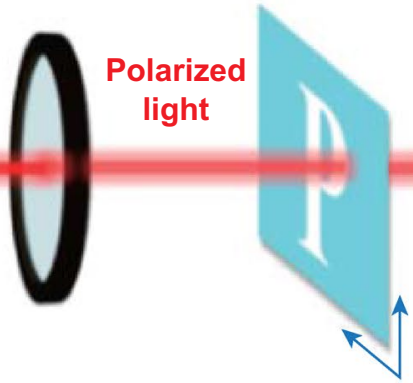

$\mathrm{X}-\mathrm{Y}$ scan
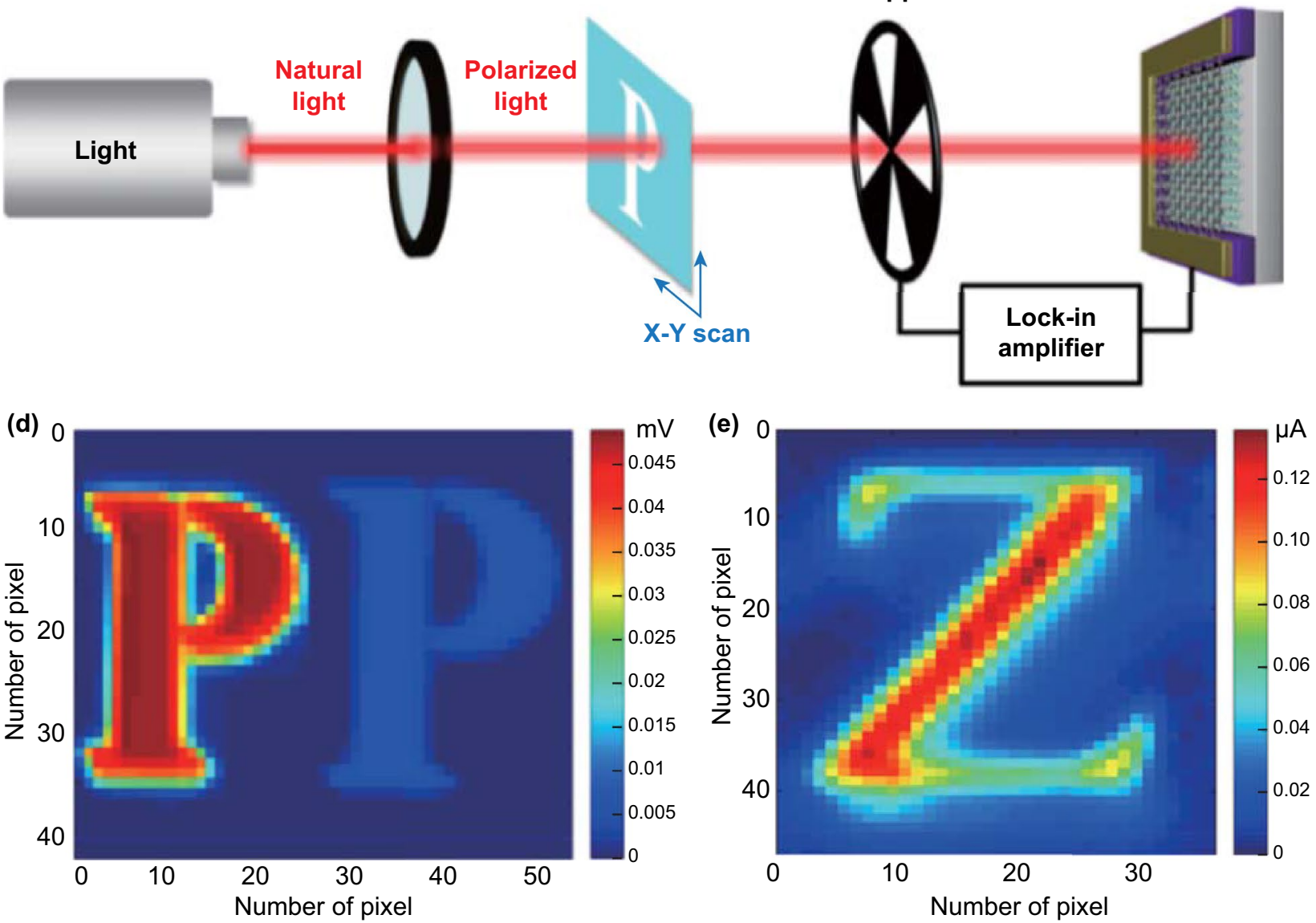

Fig. 25 Graphene/PdSe $2 / \mathrm{Ge}$ heterostructure-based polarized-light image sensor. a View of the image sensing device. b Photocurrent of the sensor under the illumination of monochromatic light by regulating different polarization angles. The light sources of four wavelengths are employed for light irradiation. c System setup for imaging the mask of a capital $P$ with the illumination of infrared polarized light. d High-resolution current mapping image of the capital $P$ under 780-nm light illumination with polarization angles of $0^{\circ}$ (left) and $90^{\circ}$ (right). e Imaging of a capital $\mathrm{Z}$ with a polarization angle of $0^{\circ}$. Reprinted with permission from Ref. [21] Copyright 2019, American Chemistry Society 
angle of $0^{\circ}$ (left panel) than that of polarized light at $90^{\circ}$ (right panel). The high polarization contrast ratio $(>10)$ between the polarization angles of $0^{\circ}$ and $90^{\circ}$ indicates the outstanding performance of the $\mathrm{PdSe}_{2}$-based device in polarized light imaging. The heterojunction-based photodetector has excellent potential as a mid-infrared image sensor. Figure $25 \mathrm{e}$ presents a highly recognizable spectral image of the $\mathrm{Z}$ letter under 3043-nm illumination with a polarization angle of $0^{\circ}$.

Such an image sensor is highly promising for broadband photodetection and imaging. The $\mathrm{PdSe}_{2}$ heterojunctionbased photodetector demonstrates an extraordinary polarization sensitivity, which is the highest value among $2 \mathrm{D}$ material-based polarized light photodetectors (thus far). On account of a strong asymmetry of $\mathrm{PdSe}_{2}$, the effective separation of photogenerated electron-hole pairs occurs by a built-in perpendicular electric field in the $p-n$ junction. Then, the efficiency of the carrier collection is enhanced by graphene electrode. Therefore, $\mathrm{PdSe}_{2}$ is a very profound material for high-performance polarization-sensitive photodetectors.

The integration with light-absorbing materials could provide the power source owing to the photovoltaic effect. Moreover, the use of perovskite as an absorber material can transform the light into electricity for self-powering by forming a Schottky junction with $\mathrm{PdSe}_{2}$. The $\mathrm{PdSe}_{2} /$ perovskite heterostructure photodetector is illustrated in Fig. 26a with a high quantum efficiency (Fig. 26b).

Such a photodetector has a broad detection band ranging from 200 to $1200 \mathrm{~nm}$. Through tuning of the illuminating light with various incident wavelengths from 265 to $980 \mathrm{~nm}$, the current of the photodetector has been recorded. Here, the photocurrent curve displays a maximum value under 808-nm illumination at a negative bias (Fig. 26c), which corresponds to the quantum efficiency peak around $800 \mathrm{~nm}$.

In the inset panel, the current-voltage curves show the photovoltaic effect upon illumination. Similarly, $\mathrm{PdSe}_{2} /$ perovskite photodetector-based arrays can be employed in image sensing with significant photoresponse capability (Fig. 26d). When the infrared light passes through the mask, the projection of the features from the mask is captured by the detector. Moreover, the processing unit converted the current signal to each pixel. Subsequently, the 2D contrast current mapping software automatically incorporates the data and exhibits the current mapping image.
Consequently, the outlines of the five letters can be recognized by $2 \mathrm{D}$ current mapping under $808-\mathrm{nm}$ illumination. Therefore, the $\mathrm{PdSe}_{2} /$ perovskite heterostructure device shows potential for the future image sensing of complicated shapes such as human beings and animals.

The currently available image sensors are listed in Table 11. The resolution and current contrast ratio may require the future efforts for improvement.

Owing to the superior capability of detecting mid-infrared light at room temperature, $\mathrm{PdSe}_{2}$-based devices highlight the high potential for application of photodetectors and image sensor systems. There remain good opportunities in the formation and application of $\mathrm{vdWHs}$ based on $\mathrm{PdSe}_{2}$ and other 2D materials. Indeed, low-dimensional materials have yet to be tested in vdWH assemblies with the coupling of $\mathrm{PdSe}_{2}$.

\section{Conclusions and Outlook}

In this work, we deliver a comprehensive review of the progress in the rising-star pentagonal 2D material, i.e., palladium diselenide. First, the fundamental of $\mathrm{PdSe}_{2}$ is introduced with the types, atomic and electronic structure, bandgap, and vibration properties. Second, the synthesis approaches are listed with top-down and bottom-up methods. Indeed, the authors are fed with mechanical exfoliation, plasma thinning, and vacuum annealing. Then, the large-area synthesis has been introduced with thermal selenization of Pd thin film, and chemical vapor deposition with different Pd precursors such as $\mathrm{PdCl}_{2}$ powers. Third, the electronic and optoelectronic devices are discussed with the metal/ semiconductor contact, field-effect transistors, photodetectors, and humidity sensors. The $\mathrm{PdSe}_{2}$ has been employed in the generation of pulsed laser and the thermoelectric power. Last but not the least, the van der Waals heterostructures of $\mathrm{PdSe}_{2}$ are delivered as well as their applications in the rectifier, photodetectors, and image system.

The fundamental physics of $\mathrm{PdSe}_{2}$ may provide for the insight for the guide of device design and fabrication. Indeed, the engineering applications of conventional devices and characterization tools require refreshing novel materials to enrich the interdisciplinary research across the microelectronics, optoelectronics, spectroscopy, optics, photonics, spintronics, and valleytronics. Besides, the magnetic properties of materials are interesting for the incubation 

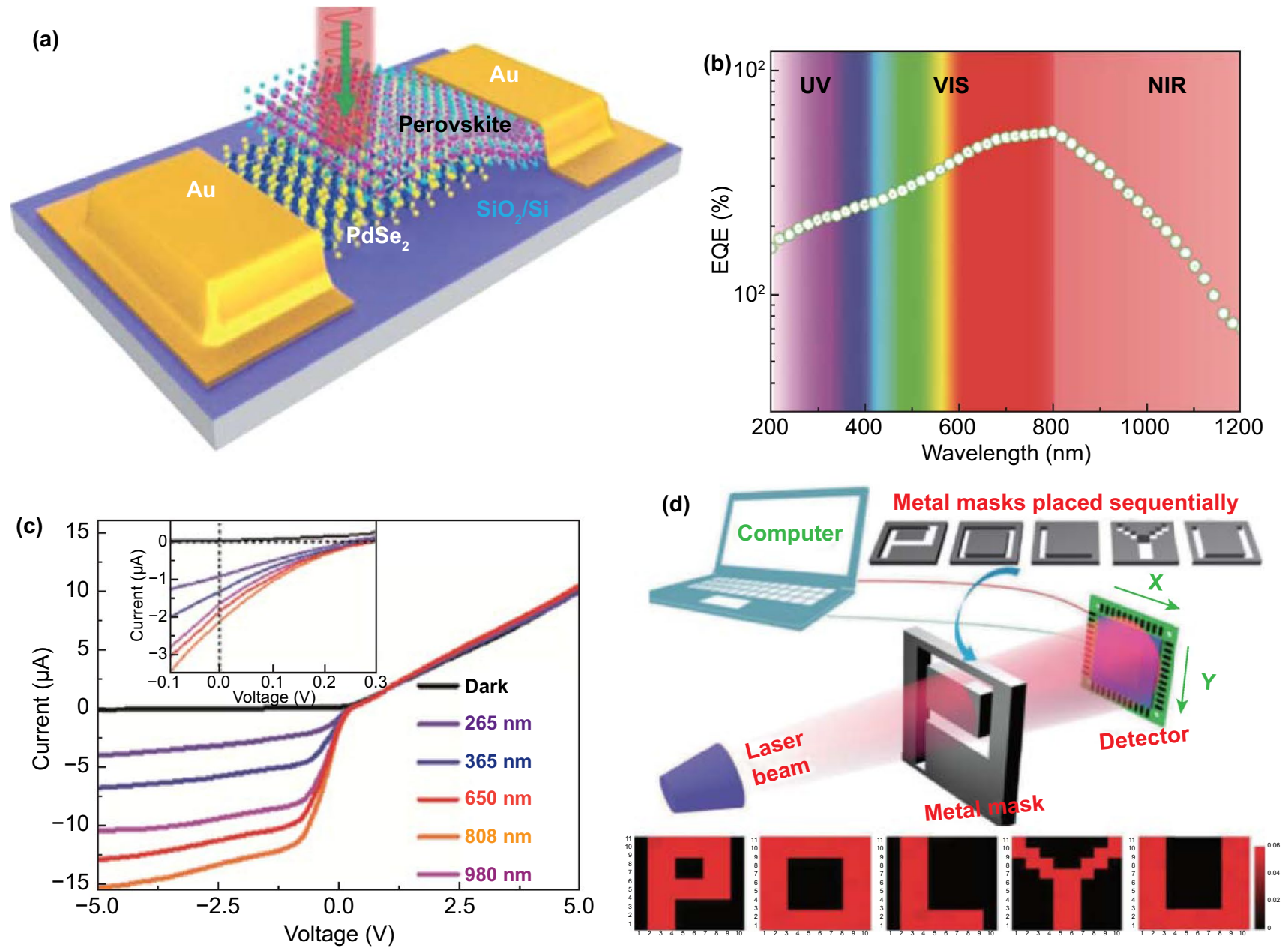

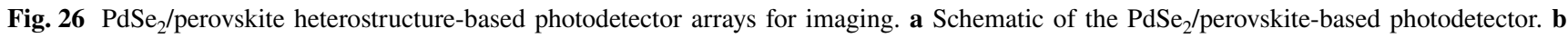
External quantum efficiency of the $\mathrm{PdSe}_{2} /$ perovskite device as a function of the incident wavelength. c Current-voltage curve of the PdSe $/$ perovskite photodetector in the dark and under illumination with different wavelengths. Inset is zoomed-in current-voltage curves at the range from -0.1 to $0.3 \mathrm{~V}$. d Scheme of the imaging system based on the photodetector arrays under 808-nm illumination, and 2D photocurrent mapping images after sensing five different letters. Adapted under the terms of the Creative Commons CC by license (https://creativecommons.org/licen ses/by/4.0/) from Ref. [26] Copyright 2019, The Authors, published by WILEY-VCH Verlag GmbH \& Co. KGaA, Weinheim

Table 11 Performances of image sensors based on $2 \mathrm{D} \mathrm{PdSe}_{2}$ and its van der Waals heterostructures

\begin{tabular}{|c|c|c|c|c|c|c|}
\hline Material types & Resolution (pixel) & Mask area $\left(\mathrm{cm}^{2}\right)$ & $\begin{array}{l}\text { Active device area } \\
\left(\mathrm{cm}^{2}\right)\end{array}$ & $\begin{array}{l}\text { Current contrast } \\
\text { ratio }\end{array}$ & $\begin{array}{l}\text { Wavelength } \lambda \\
(\mathrm{nm})\end{array}$ & $\begin{array}{l}\text { Refer- } \\
\text { ences }\end{array}$ \\
\hline $\mathrm{PdSe}_{2} / \mathrm{Ge}$ & $46 \times 46$ & $5 \times 5$ & $0.6 \times 0.6$ & $>10^{2}$ & 3043 & [21] \\
\hline $\mathrm{PdSe}_{2} /$ pyramid Si & $19 \times 20$ & several & $0.1 \times 0.1$ & $>10^{2}$ & 1330 & [119] \\
\hline $\mathrm{PdSe}_{2} / \mathrm{GeNCs}$ & $6 \times 6$ & several & Sub-1 & $10^{2}$ & 1550 & [87] \\
\hline
\end{tabular}

of the proof-of-concept devices. Besides, the band alignment in a heterostructure may provide a platform for photogenerated carrier transport. The 2D materials as saturable absorbers have demonstrated extraordinary performances in Q-switching and mode lock for pulsed laser generation. Indeed, the metallic 2D materials have demonstrated superior performances in electromagnetic interference shielding or microwave absorption. Besides, the incorporation of 
magnetic nanoparticles may lead to the change of magnetoresistance as a magnetic field sensor.

The performance of $\mathrm{PdSe}_{2}$ devices has been verified in photodetectors[62], field-effect transistors [16], and humidity sensors [25]. First, $\mathrm{PdSe}_{2}$ transistors demonstrate pressure-tunable hysteresis [70], field emission [81], and phototransistors [41]. Second, the narrow bandgap of $\mathrm{PdSe}_{2}$ guarantees its performance in an infrared range such as 10.6$\mu \mathrm{m}$ light detection [54] and broadband sensing [19]. Third, the linear dichroism transition [39] in $\mathrm{PdSe}_{2}$ guarantees optical switching and communication. As a saturable absorber, $\mathrm{PdSe}_{2}$ shows success in Q-switching for pulsed lasers [96].

There are still plenty of room in the development of sophisticated techniques for mass production of $\mathrm{PdSe}_{2}$. First, the chemical vapor deposition [120] has the features of upscale production, large-area homogeneity, and compatibility with Si-based technology. Indeed, the chemical vapor deposition of 2D materials [121-123] become necessary to achieve the synthesis over a large area and even a wafer size [124]. Owing to the layer-dependent properties, the preparation methods of high-quality $2 \mathrm{D} \mathrm{PdSe} \mathrm{Pe}_{2}$ should be modified to accurately control the thickness, which is essential for the manufacture of high-performance devices. According to the trend of sophisticated 2D materials such as graphene, the quality of synthetic $\mathrm{PdSe}_{2}$ may go through the path, i.e., from mechanically exfoliated nanosheets, ball-milled nanopowders [125], polycrystalline thick films, monolayer or bilayer polycrystalline thin film [126, 127], and monolayer single-crystal domains [128]. More effective synthesis of atomically thin, large-scale, and uniform $2 \mathrm{D} \mathrm{PdSe}{ }_{2}$ should be explored to satisfy the needs of industrialization. Eventually, the domain size of $\mathrm{PdSe}_{2}$ single crystal may expand to centimeter scale and even to the wafer scale.

The posttreatment of $\mathrm{PdSe}_{2}$ may broaden its material properties, and consequently, its device performances may vary. First, the thermal annealing or plasma treatment [129] has shown modification of 2D materials. Second, the in situ characterization tools such as transmission electron microscopy [130] and XRD may provide the direct evidence for phase transition, i.e., the lattice distortion in the atomic scale. Third, the machine learning acts an efficient tool for defects determination and device performances enhancement. The properties of 2D materials could be regulated with defect engineering by the theoretical calculation, as well as big data for materials science. Besides, the patterning of $2 \mathrm{D}$ materials becomes a prerequisite for the fabrication of device arrays.

Future opportunities of $\mathrm{PdSe}_{2}$-based devices and systems remain great at integrated circuits as well as the internet of things. Indeed, the 2D materials have been incorporated in the logic gate-based digital circuits, programmable memories, and RF integrated circuits. One can refer to the graphene [124] and transition-metal dichalcogenides [131] for borrowing the concept of heterostructures. There remains a vortex of materials science research for artificial intelligence such as actuator devices, and human/machine interface. Therefore, great prospects of $\mathrm{PdSe}_{2}$-based van der Waals heterostructures are calling for the input of physicists, chemists, and materials scientists as well as industrial engineers.

Acknowledgements H.L. acknowledges the National Key Research and Development Program of China (2017YFB0405400) from the Ministry of Science and Technology (MOST) of China, and the Natural Science Foundation for Distinguished Young Scientist of Shandong Province (Grant No. JQ201814). We thank the Project of "20 items of University" of Jinan (2018GXRC031). W.Z thanks Taishan Scholars Project Special Funds (tsqn201812083) and NSFC (No. 52022037). The authors show their gratitude to the National Natural Science Foundation of China (NSFC grant No. 51802113, 51802116) and the Natural Science Foundation of Shandong Province, China (grant No. ZR2019BEM040, ZR2018BEM015). M.H.R. thanks the National Science Foundation China (NSFC, Project 52071225), the National Science Center and the Czech Republic under the ERDF program "Institute of Environmental Technology_Excellent Research" (No. CZ.02.1.01/0.0 /0.0/16_019/0000853) and the Sino-German Research Institute for support (Project No. GZ 1400).

Open Access This article is licensed under a Creative Commons Attribution 4.0 International License, which permits use, sharing, adaptation, distribution and reproduction in any medium or format, as long as you give appropriate credit to the original author(s) and the source, provide a link to the Creative Commons licence, and indicate if changes were made. The images or other third party material in this article are included in the article's Creative Commons licence, unless indicated otherwise in a credit line to the material. If material is not included in the article's Creative Commons licence and your intended use is not permitted by statutory regulation or exceeds the permitted use, you will need to obtain permission directly from the copyright holder. To view a copy of this licence, visit http://creativecommons.org/licenses/by/4.0/.

\section{References}

1. Y. Saito, J. Ge, K. Watanabe, T. Taniguchi, A.F. Young, Independent superconductors and correlated insulators in twisted 
bilayer graphene. Nat. Phys. 16(9), 926-930 (2020). https:// doi.org/10.1038/s41567-020-0928-3

2. C. Jin, J. Kim, M.I.B. Utama, E.C. Regan, H. Kleemann et al., Imaging of pure spin-valley diffusion current in $\mathrm{WS}_{2}-\mathrm{WSe}_{2}$ heterostructures. Science 360(6391), 893-896 (2018). https:// doi.org/10.1126/science.aao3503

3. Y. Pang, Z. Yang, Y. Yang, T.L. Ren, Wearable electronics based on 2D materials for human physiological information detection. Small 16(15), 1901124 (2020). https://doi.org/10. 1002/smll.201901124

4. A.V. Agrawal, N. Kumar, M. Kumar, Strategy and future prospects to develop room-temperature-recoverable $\mathrm{NO}_{2}$ gas sensor based on two-dimensional molybdenum disulfide. Nano-Micro Lett. 13(1), 38 (2021). https://doi.org/10.1007/ s40820-020-00558-3

5. N.E. Holden, T.B. Coplen, J.K. Böhlke, L.V. Tarbox, J. Benefield et al., IUPAC periodic table of the elements and isotopes (IPTEI) for the education community (IUPAC Technical Report). Pure Appl. Chem. 90(12), 1833-2092 (2018). https://doi.org/10.1515/pac-2015-0703

6. K.F. Mak, J. Shan, Photonics and optoelectronics of 2D semiconductor transition metal dichalcogenides. Nat. Photon. 10(4), 216-226 (2016). https://doi.org/10.1038/nphoton. 2015.282

7. L. Zeng, S. Lin, Z. Lou, H. Yuan, H. Long et al., Ultrafast and sensitive photodetector based on a $\mathrm{PtSe}_{2} /$ silicon nanowire array heterojunction with a multiband spectral response from 200 to $1550 \mathrm{~nm}$. NPG Asia Mater. 10(4), 352-362 (2018). https://doi.org/10.1038/s41427-018-0035-4

8. R. Kempt, A. Kuc, T. Heine, Two-dimensional noble-metal chalcogenides and phosphochalcogenides. Angew. Chem. Int. Ed. 59(24), 9242-9254 (2020). https://doi.org/10.1002/anie. 201914886

9. S. Ahmad, Strain dependent tuning electronic properties of noble metal di chalcogenides $\mathrm{PdX}_{2}(\mathrm{X}=\mathrm{S}$, Se) mono-layer. Mater. Chem. Phys. 198(1), 162-166 (2017). https://doi.org/ 10.1016/j.matchemphys.2017.05.060

10. L. Zeng, D. Wu, J. Jie, X. Ren, X. Hu et al., Van der Waals epitaxial growth of mosaic-like 2D platinum ditelluride layers for room-temperature mid-infrared photodetection up to 10.6 microm. Adv. Mater. 32(52), 2004412 (2020). https://doi.org/ 10.1002/adma.202004412

11. Y. Zhao, J. Qiao, Z. Yu, P. Yu, K. Xu et al., High-electronmobility and air-stable 2D layered PtSe ${ }_{2}$ FETs. Adv. Mater. 29(5), 1604230 (2017). https://doi.org/10.1002/adma.20160 4230

12. H. Yang, Y. Li, Z. Yang, X. Shi, Z. Lin et al., First-principles calculations of the electronic properties of two-dimensional pentagonal structure $\mathrm{XS}_{2}(\mathrm{X}=\mathrm{Ni}, \mathrm{Pd}, \mathrm{Pt})$. Vacuum 174(1), 109176 (2020). https://doi.org/10.1016/j.vacuum. 2020.109176

13. D. Saraf, S. Chakraborty, A. Kshirsagar, R. Ahuja, In pursuit of bifunctional catalytic activity in PdS2 pseudo-monolayer through reaction coordinate mapping. Nano Energy
49(4), 283-289 (2018). https://doi.org/10.1016/j.nanoen. 2018.04.019

14. M. Ghorbani-Asl, A. Kuc, P. Miro, T. Heine, A singlematerial logical junction based on 2D Crystal $\mathrm{PdS}_{2}$. Adv. Mater. 28(5), 853-856 (2016). https://doi.org/10.1002/ adma.201504274

15. A.D. Oyedele, S. Yang, L. Liang, A.A. Puretzky, K. Wang et al., $\mathrm{PdSe}_{2}$ : pentagonal two-dimensional layers with high air stability for electronics. J. Am. Chem. Soc. 139(40), 14090-14097 (2017). https://doi.org/10.1021/jacs.7b04865

16. Y. Gu, H. Cai, J. Dong, Y. Yu, A.N. Hoffman et al., Twodimensional palladium diselenide with strong in-plane optical anisotropy and high mobility grown by chemical vapor deposition. Adv. Mater. 32(19), 1906238 (2020). https:// doi.org/10.1002/adma.201906238

17. W.L. Chow, P. Yu, F. Liu, J. Hong, X. Wang et al., High mobility 2D palladium diselenide field-effect transistors with tunable ambipolar characteristics. Adv. Mater. 29(21), 1602969 (2017). https://doi.org/10.1002/adma.201602969

18. A.A. Puretzky, A.D. Oyedele, K. Xiao, A.V. Haglund, B.G. Sumpter et al., Anomalous interlayer vibrations in strongly coupled layered $\mathrm{PdSe}_{2}$. 2D Mater. 5(3), 35016 (2018). https://doi.org/10.1088/2053-1583/aabe4d

19. Q. Liang, Q. Wang, Q. Zhang, J. Wei, S.X. Lim et al., Highperformance, room temperature, ultra-broadband photodetectors based on air-stable PdSe ${ }_{2}$. Adv. Mater. 31(24), 1807609 (2019). https://doi.org/10.1002/adma.201807609

20. H. Yang, S.W. Kim, M. Chhowalla, Y.H. Lee, Structural and quantum-state phase transitions in van der Waals layered materials. Nat. Phys. 13(10), 931-937 (2017). https:// doi.org/10.1038/nphys4188

21. D. Wu, J. Guo, J. Du, C. Xia, L. Zeng et al., Highly polarization-sensitive, broadband, self-powered photodetector based on graphene/ $\mathrm{PdSe}_{2}$ /germanium heterojunction. ACS Nano 13(9), 9907-9917 (2019). https://doi.org/10.1021/ acsnano.9b03994

22. K.L. Tai, J. Chen, Y. Wen, H. Park, Q. Zhang et al., Phase variations and layer epitaxy of $2 \mathrm{D} \mathrm{PdSe}_{2}$ GRown on 2D monolayers by direct selenization of molecular Pd precursors. ACS Nano 14(9), 11677-11690 (2020). https://doi. org/10.1021/acsnano.0c04230

23. M. Jakhar, J. Singh, A. Kumar, K. Tankeshwar, Pressure and electric field tuning of Schottky contacts in $\mathrm{PdSe}_{2} / \mathrm{ZT}$ $\mathrm{MoSe}_{2}$ van der Waals heterostructure. Nanotechnology 31(14), 145710 (2020). https://doi.org/10.1088/1361-6528/ ab5de 1

24. A.M. Afzal, M.Z. Iqbal, S. Mumtaz, I. Akhtar, Multifunctional and high-performance GeSe/PdSe2 heterostructure device with a fast photoresponse. J. Mater. Chem. C 8(14), 4743-4753 (2020). https://doi.org/10.1039/d0tc00004c

25. D. Wu, C. Jia, F. Shi, L. Zeng, P. Lin et al., Mixed-dimensional $\mathrm{PdSe}_{2} / \mathrm{SiNWA}_{\mathrm{N}}$ heterostructure based photovoltaic detectors for self-driven, broadband photodetection, infrared imaging and humidity sensing. J. Mater. Chem. A 8(7), 3632-3642 (2020). https://doi.org/10.1039/c9ta13611h 
26. L.H. Zeng, Q.M. Chen, Z.X. Zhang, D. Wu, H. Yuan et al., Multilayered $\mathrm{PdSe}_{2} /$ perovskite schottky junction for fast, self-powered, polarization-sensitive, broadband photodetectors, and image sensor application. Adv. Sci. 6(19), 1901134 (2019). https://doi.org/10.1002/advs.201901134

27. J. Sun, H. Shi, T. Siegrist, D.J. Singh, Electronic, transport, and optical properties of bulk and mono-layer $\mathrm{PdSe}_{2}$. Appl. Phys. Lett. 107(15), 153902 (2015). https://doi.org/10. 1063/1.4933302

28. F. Grønvold, E. Røst, The crystal structure of $\mathrm{PdSe}_{2}$ and $\mathrm{PdS}_{2}$. Acta Crystallogr. 10(4), 329-331 (1957). https://doi.org/10. 1107/s0365110x57000948

29. J. Zhong, J. Yu, L. Cao, C. Zeng, J. Ding et al., High-performance polarization-sensitive photodetector based on a few-layered $\mathrm{PdSe}_{2}$ nanosheet. Nano Res. 13(6), 1780-1786 (2020). https://doi.org/10.1007/s12274-020-2804-y

30. Y. Zhao, J. Qiao, P. Yu, Z. Hu, Z. Lin et al., Extraordinarily strong interlayer interaction in $2 \mathrm{D}$ layered $\mathrm{PtS}_{2}$. Adv. Mater. 28(12), 2399-2407 (2016). https://doi.org/10.1002/adma. 201504572

31. A.V. Kuklin, H. Ågren, Quasiparticle electronic structure and optical spectra of single-layer and bilayer $\mathrm{PdSe}_{2}$ : Proximity and defect-induced band gap renormalization. Phys. Rev. B 99(24), 2469-9950 (2019). https://doi.org/10.1103/PhysR evB.99.245114

32. X. Zhao, Q. Zhao, B. Zhao, X. Dai, S. Wei et al., Electronic and optical properties of $\mathrm{PdSe}_{2}$ from monolayer to trilayer. Superlattices Microstr. 142(4), 106514 (2020). https://doi. org/10.1016/j.spmi.2020.106514

33. W. Lei, B. Cai, H. Zhou, G. Heymann, X. Tang et al., Ferroelastic lattice rotation and band-gap engineering in quasi 2D layered-structure $\mathrm{PdSe}_{2}$ under uniaxial stress. Nanoscale 11(25), 12317-12325 (2019). https://doi.org/10.1039/c9nr0 $3101 \mathrm{~d}$

34. X. Zhao, B. Qiu, G. Hu, W. Yue, J. Ren et al., Spin polarization properties of pentagonal $\mathrm{PdSe}(2)$ induced by $3 \mathrm{D}$ transition-metal doping: first-principles calculations. Materials 11(11), 2339 (2018). https://doi.org/10.3390/ma11112339

35. S.-H. Zhang, B.-G. Liu, Hole-doping-induced half-metallic ferromagnetism in a highly-air-stable PdSe 2 monolayer under uniaxial stress. J. Mater. Chem. C 6(25), 6792-6798 (2018). https://doi.org/10.1039/c8tc01450g

36. S. Deng, L. Li, Y. Zhang, Strain modulated electronic, mechanical, and optical properties of the monolayer $\mathrm{PdS}_{2}$, $\mathrm{PdSe}_{2}$, and $\mathrm{PtSe}_{2}$ for tunable devices. ACS Appl. Nano Mater. 1(4), 1932-1939 (2018). https://doi.org/10.1021/acsanm. $8 \mathrm{~b} 00363$

37. G. Liu, Q.M. Zeng, P.F. Zhu, R.G. Quhe, P.F. Lu, Negative Poisson's ratio in monolayer $\mathrm{PdSe}_{2}$. Comput. Mater. Sci. 160(1), 309-314 (2019). https://doi.org/10.1016/j.comma tsci.2019.01.024

38. M.A. ElGhazali, P.G. Naumov, H. Mirhosseini, V. Suss, L. Muchler et al., Pressure-induced superconductivity up to 13.1 $\mathrm{K}$ in the pyrite phase of palladium diselenide PdSe2. Phys. Rev. B 96(6), 060509 (2017). https://doi.org/10.1103/PhysR evB.96.060509
39. J. Yu, X. Kuang, Y. Gao, Y. Wang, K. Chen et al., Direct observation of the linear dichroism transition in two-dimensional palladium diselenide. Nano Lett. 20(2), 1172-1182 (2020). https://doi.org/10.1021/acs.nanolett.9b04598

40. W. Lei, S. Zhang, G. Heymann, X. Tang, J. Wen et al., A new $2 \mathrm{D}$ high-pressure phase of $\mathrm{PdSe}_{2}$ with high-mobility transport anisotropy for photovoltaic applications. J. Mater. Chem. C 7(7), 2096-2105 (2019). https://doi.org/10.1039/c8tc06050a

41. T.S. Walmsley, K. Andrews, T. Wang, A. Haglund, U. Rijal et al., Near-infrared optical transitions in $\mathrm{PdSe}_{2}$ phototransistors. Nanoscale 11(30), 14410-14416 (2019). https://doi.org/ $10.1039 / \mathrm{c} 9 \mathrm{nr} 03505 \mathrm{~b}$

42. M. Sun, J.P. Chou, L. Shi, J. Gao, A. Hu et al., Few-Layer $\mathrm{PdSe}_{2}$ sheets: promising thermoelectric materials driven by high valley convergence. ACS Omega 3(6), 5971-5979 (2018). https://doi.org/10.1021/acsomega.8b00485

43. Y. Cai, G. Zhang, Y.W. Zhang, Polarity-reversed robust carrier mobility in monolayer $\operatorname{MoS}(2)$ nanoribbons. J. Am. Chem. Soc. 136(17), 6269-6275 (2014). https://doi.org/10. 1021/ja4109787

44. X.-J. Ge, D. Qin, K.-L. Yao, J.-T. Lü, First-principles study of thermoelectric transport properties of monolayer gallium chalcogenides. J. Phys. D-Appl. Phys. 50(40), 405301 (2017). https://doi.org/10.1088/1361-6463/aa85b4

45. G.D. Nguyen, L. Liang, Q. Zou, M. Fu, A.D. Oyedele et al., 3D imaging and manipulation of subsurface selenium vacancies in $\mathrm{PdSe}_{2}$. Phys. Rev. Lett. 121(8), 086101 (2018). https:// doi.org/10.1103/PhysRevLett.121.086101

46. J. Lin, S. Zuluaga, P. Yu, Z. Liu, S.T. Pantelides et al., Novel $\mathrm{Pd}_{2} \mathrm{Se}_{3}$ two-dimensional phase driven by interlayer fusion in layered $\mathrm{PdSe}_{2}$. Phys. Rev. Lett. 119(1), 016101 (2017). https://doi.org/10.1103/PhysRevLett.119.016101

47. J. Chen, G.H. Ryu, S. Sinha, J.H. Warner, Atomic structure and dynamics of defects and grain boundaries in $2 \mathrm{D} \mathrm{Pd}_{2} \mathrm{Se}_{3}$ Monolayers. ACS Nano 13(7), 8256-8264 (2019). https://doi. org/10.1021/acsnano.9b03645

48. S. Zuluaga, J. Lin, K. Suenaga, S.T. Pantelides, Two-dimensional $\mathrm{PdSe}_{2}-\mathrm{Pd}_{2} \mathrm{Se}_{3}$ junctions can serve as nanowires. 2D Mater. 5(3), 035025 (2018). https://doi.org/10.1088/2053$1583 / \mathrm{aac} 34 \mathrm{c}$

49. G.H. Ryu, T. Zhu, J. Chen, S. Sinha, V. Shautsova, Striated 2D lattice with sub-nm $1 \mathrm{D}$ etch channels by controlled thermally induced phase transformations of $\mathrm{PdSe}_{2}$. Adv. Mater. 31(46), 1904251 (2019). https://doi.org/10.1002/adma.20190 4251

50. V. Shautsova, S. Sinha, L. Hou, Q. Zhang, M. Tweedie et al., Direct laser patterning and phase transformation of $2 \mathrm{D} \mathrm{PdSe} \mathrm{P}_{2}$ films for on-demand device fabrication. ACS Nano 13(12), 14162-14171 (2019). https://doi.org/10.1021/acsnano.9b068 92

51. T. Takabatake, M. Ishikawa, J.L. Jorda, Superconductivity and phase relations in the Pd-Se system. J. Less Common Met. 134(1), 79-89 (1987). https://doi.org/10.1016/00225088(87)90444-9

52. A.D. Oyedele, S. Yang, T. Feng, A.V. Haglund, Y. Gu et al., Defect-mediated phase transformation in anisotropic 
two-dimensional $\mathrm{PdSe}_{2}$ crystals for seamless electrical contacts. J. Am. Chem. Soc. 141(22), 8928-8936 (2019). https:// doi.org/10.1021/jacs.9b02593

53. D. Wang, F. Luo, M. Lu, X. Xie, L. Huang et al., Chemical vapor transport reactions for synthesizing layered materials and their 2D counterparts. Small 15(40), 1804404 (2019). https://doi.org/10.1002/smll.201804404

54. M. Long, Y. Wang, P. Wang, X. Zhou, H. Xia et al., Palladium diselenide long-wavelength infrared photodetector with high sensitivity and stability. ACS Nano 13(2), 2511-2519 (2019). https://doi.org/10.1021/acsnano.8b09476

55. M. Velicky, G.E. Donnelly, W.R. Hendren, S. McFarland, D. Scullion et al., Mechanism of gold-assisted exfoliation of centimeter-sized transition-metal dichalcogenide monolayers. ACS Nano 12(10), 10463-10472 (2018). https://doi.org/10. 1021/acsnano.8b06101

56. M. Heyl, D. Burmeister, T. Schultz, S. Pallasch, G. Ligorio et al., Thermally activated gold-mediated transition metal dichalcogenide exfoliation and a unique gold-mediated transfer. Phys. Status Solidi (RRL) 14(11), 2000408 (2020). https://doi.org/10.1002/pssr.202000408

57. S.B. Desai, S.R. Madhvapathy, M. Amani, D. Kiriya, M. Hettick et al., Gold-mediated exfoliation of ultralarge optoelectronically-perfect monolayers. Adv. Mater. 28(21), 40534058 (2016). https://doi.org/10.1002/adma.201506171

58. Y. Huang, Y.H. Pan, R. Yang, L.H. Bao, L. Meng et al., Universal mechanical exfoliation of large-area 2D crystals. Nat. Commun. 11(1), 2453 (2020). https://doi.org/10.1038/ s41467-020-16266-w

59. D. Zhao, S. Xie, Y. Wang, H. Zhu, L. Chen et al., Synthesis of large-scale few-layer $\mathrm{PtS}_{2}$ films by chemical vapor deposition. AIP Adv. 9(2), 025225 (2019). https://doi.org/10.1063/1. 5086447

60. L. Jia, J. Wu, T. Yang, B. Jia, D.J. Moss, Large third-order optical kerr nonlinearity in nanometer-thick $\mathrm{PdSe}_{2} 2 \mathrm{D}$ dichalcogenide films: implications for nonlinear photonic devices. ACS Appl. Nano Mater. 3(7), 6876-6883 (2020). https://doi. org/10.1021/acsanm.0c01239

61. J. Zhou, J. Lin, X. Huang, Y. Zhou, Y. Chen et al., A library of atomically thin metal chalcogenides. Nature 556(7701), 355-359 (2018). https://doi.org/10.1038/s41586-018-0008-3

62. L.H. Zeng, D. Wu, S.H. Lin, C. Xie, H.Y. Yuan et al., Controlled synthesis of $2 \mathrm{D}$ palladium diselenide for sensitive photodetector applications. Adv. Funct. Mater. 29(1), 1806878 (2019). https://doi.org/10.1002/adfm.201806878

63. L.S. Lu, G.H. Chen, H.Y. Cheng, C.P. Chuu, K.C. Lu et al., Layer-dependent and in-plane anisotropic properties of lowtemperature synthesized few-layer $\mathrm{PdSe}_{2}$ single crystals. ACS Nano 14(4), 4963-4972 (2020). https://doi.org/10.1021/acsna no.0c01139

64. G.D. Nguyen, A.D. Oyedele, A. Haglund, W. Ko, L. Liang et al., Atomically precise $\mathrm{PdSe} \mathrm{S}_{2}$ pentagonal nanoribbons. ACS Nano 14(2), 1951-1957 (2020). https://doi.org/10.1021/ acsnano.9b08390

65. L.H. Zeng, S.H. Lin, Z.J. Li, Z.X. Zhang, T.F. Zhang et al., Fast, self-driven, air-stable, and broadband photodetector based on vertically aligned $\mathrm{PtSe}_{2} / \mathrm{GaAs}$ heterojunction. Adv. Funct. Mater. 28(16), 1705970 (2018). https://doi.org/10. 1002/adfm.201705970

66. A.N. Hoffman, Y. Gu, J. Tokash, J. Woodward, K. Xiao et al., Layer-by-layer thinning of pdse2 flakes via plasma induced oxidation and sublimation. ACS Appl. Mater. Interfaces 12(6), 7345-7350 (2020). https://doi.org/10.1021/acsami. $9 b 21287$

67. Q. Liang, Q. Zhang, J. Gou, T. Song, Arramel et al., Performance improvement by ozone treatment of $2 \mathrm{D} \mathrm{PdSe}{ }_{2}$. ACS Nano 14(5), 5668-5677 (2020). https://doi.org/10.1021/acsna no.0c00180

68. A. Di. Bartolomeo, F. Urban, A. Pelella, A. Grillo, M. Passacantando et al., Electron irradiation of multilayer $\mathrm{PdSe}_{2}$ field effect transistors. Nanotechnology 31(37), 375204 (2020). https://doi.org/10.1088/1361-6528/ab9472

69. A. Hassan, Y. Guo, Q. Wang, Performance of the pentagonal $\mathrm{PdSe}_{2}$ sheet as a channel material in contact with metal surfaces and graphene. ACS Appl. Electron. Mater. 2(8), 2535-2542 (2020). https://doi.org/10.1021/acsaelm.0c00438

70. A. Di. Bartolomeo, A. Pelella, X. Liu, F. Miao, M. Passacantando et al., Pressure-tunable ambipolar conduction and hysteresis in thin palladium diselenide field effect transistors. Adv. Funct. Mater. 29(29), 1902483 (2019). https://doi.org/ 10.1002/adfm.201902483

71. J. Gao, Y. Gao, Y. Han, J. Pang, C. Wang et al., Ultrasensitive label-free MiRNA sensing based on a flexible graphene field-effect transistor without functionalization. ACS Appl. Electron. Mater. 2(4), 1090-1098 (2020). https://doi.org/10. 1021/acsaelm.0c00095

72. A. Tankut, M. Karaman, I. Yildiz, S. Canli, R. Turan, Effect of Al vacuum annealing prior to a-Si deposition on aluminum-induced crystallization. Phys. Status Solidi A Appl. Mater. Sci. 212(12), 2702-2707 (2015). https://doi.org/10. 1002/pssa.201532857

73. T. Takenobu, T. Kanbara, N. Akima, T. Takahashi, M. Shiraishi et al., Control of carrier density by a solution method in carbon-nanotube devices. Adv. Mater. 17(20), 2430-2434 (2005). https://doi.org/10.1002/adma.200500759

74. F. Giubileo, A. Grillo, L. Iemmo, G. Luongo, F. Urban et al., Environmental effects on transport properties of $\mathrm{PdSe}_{2}$ field effect transistors. Mater. Today Proc. 20(1), 50-53 (2020). https://doi.org/10.1016/j.matpr.2019.08.226

75. G.T. Xia, Y.N. Huang, F.J. Li, L.C. Wang, J.B. Pang et al., A thermally flexible and multi-site tactile sensor for remote 3D dynamic sensing imaging. Front. Chem. Sci. Eng. 14(6), 1039-1051 (2020). https://doi.org/10.1007/ s11705-019-1901-5

76. D. Chen, Z. Liu, Y. Li, D. Sun, X. Liu et al., Unsymmetrical alveolate PMMA/MWCNT film as a piezoresistive E-skin with four-dimensional resolution and application for detecting motion direction and airflow rate. ACS Appl. Mater. Interfaces 12(27), 30896-30904 (2020). https://doi.org/10. 1021/acsami.0c02640

77. Y. Zhou, Y. Wang, K. Wang, L. Kang, F. Peng et al., Hybrid genetic algorithm method for efficient and robust evaluation 
of remaining useful life of supercapacitors. Appl. Energy 260(1), 114169 (2020). https://doi.org/10.1016/j.apenergy. 2019.114169

78. X. Shang, S. Li, K. Wang, X. Teng, X. Wang et al., $\mathrm{MnSe}_{2} /$ Se composite nanobelts as an improved performance anode for lithium storage. Int. J. Electrochem. Sci. 14(1), 60006008 (2019). https://doi.org/10.20964/2019.07.37

79. C. Bu, F. Li, K. Yin, J. Pang, L. Wang et al., Research progress and prospect of triboelectric nanogenerators as self-powered human body sensors. ACS Appl. Electron. Mater. 2(4), 863-878 (2020). https://doi.org/10.1021/acsae $\operatorname{lm} .0 \mathrm{c} 00022$

80. S.C. Dhanabalan, J.S. Ponraj, H. Zhang, Q. Bao, Present perspectives of broadband photodetectors based on nanobelts, nanoribbons, nanosheets and the emerging 2D materials. Nanoscale 8(12), 6410-6434 (2016). https://doi.org/ 10.1039/c5nr09111j

81. A. Di. Bartolomeo, A. Pelella, F. Urban, A. Grillo, L. Iemmo et al., Field emission in ultrathin $\mathrm{PdSe}_{2}$ back-gated transistors. Adv. Electron. Mater. 6(7), 2000094 (2020). https://doi.org/10.1002/aelm.202000094

82. R. Zhuo, L. Zeng, H. Yuan, D. Wu, Y. Wang et al., In-situ fabrication of $\mathrm{PtSe}_{2} / \mathrm{GaN}$ heterojunction for self-powered deep ultraviolet photodetector with ultrahigh current on/ off ratio and detectivity. Nano Res. 12(1), 183-189 (2018). https://doi.org/10.1007/s12274-018-2200-z

83. M. Buscema, D.J. Groenendijk, S.I. Blanter, G.A. Steele, H.S. van der Zant et al., Fast and broadband photoresponse of few-layer black phosphorus field-effect transistors. Nano Lett. 14(6), 3347-3352 (2014). https://doi.org/10.1021/ nl5008085

84. X. Wan, Y. Xu, H. Guo, K. Shehzad, A. Ali et al., A selfpowered high-performance graphene/silicon ultraviolet photodetector with ultra-shallow junction: breaking the limit of silicon? NPJ 2D Mater. Appl. 1(1), 2397-7132 (2017). https://doi.org/10.1038/s41699-017-0008-4

85. R. Zhuo, Y. Wang, D. Wu, Z. Lou, Z. Shi et al., Highperformance self-powered deep ultraviolet photodetector based on $\mathrm{MoS}_{2} / \mathrm{GaN}$ p-n heterojunction. J. Mater. Chem. C 6(2), 299-303 (2018). https://doi.org/10.1039/c7tc04754a

86. E.P. Mukhokosi, S.B. Krupanidhi, K.K. Nanda, Band gap engineering of hexagonal $\mathrm{SnSe}_{2}$ nanostructured thin films for infra-red photodetection. Sci. Rep. 7(1), 15215 (2017). https://doi.org/10.1038/s41598-017-15519-x

87. L.B. Luo, D. Wang, C. Xie, J.G. Hu, X.Y. Zhao et al., PdSe ${ }_{2}$ multilayer on germanium nanocones array with light trapping effect for sensitive infrared photodetector and image sensing application. Adv. Funct. Mater. 29(22), 1900849 (2019). https://doi.org/10.1002/adfm.201900849

88. Y. Yang, S.C. Liu, X. Wang, Z. Li, Y. Zhang et al., Polarization-sensitive ultraviolet photodetection of anisotropic 2D $\mathrm{GeS}_{2}$. Adv. Funct. Mater. 29(16), 1900411 (2019). https://doi. org/10.1002/adfm.201900411

89. F. Chu, M. Chen, Y. Wang, Y. Xie, B. Liu et al., A highly polarization sensitive antimonene photodetector with a broadband photoresponse and strong anisotropy. J. Mater. Chem. C 6(10), 2509-2514 (2018). https://doi.org/10.1039/ $\mathrm{c} 7 \mathrm{tc} 05488 \mathrm{~b}$

90. P.K. Venuthurumilli, P.D. Ye, X. Xu, Plasmonic Resonance enhanced polarization-sensitive photodetection by black phosphorus in near infrared. ACS Nano 12(5), 4861-4867 (2018). https://doi.org/10.1021/acsnano.8b01660

91. Y. Yang, S.C. Liu, W. Yang, Z. Li, Y. Wang et al., Air-stable in-plane anisotropic $\mathrm{GeSe}_{2}$ for highly polarization-sensitive photodetection in short wave region. J. Am. Chem. Soc. 140(11), 4150-4156 (2018). https://doi.org/10.1021/jacs. $8 \mathrm{~b} 01234$

92. J. Bullock, M. Amani, J. Cho, Y.-Z. Chen, G.H. Ahn et al., Polarization-resolved black phosphorus/molybdenum disulfide mid-wave infrared photodiodes with high detectivity at room temperature. Nat. Photon. 12(10), 601-607 (2018). https://doi.org/10.1038/s41566-018-0239-8

93. J. Du, M. Zhang, Z. Guo, J. Chen, X. Zhu et al., Phosphorene quantum dot saturable absorbers for ultrafast fiber lasers. Sci. Rep. 7(1), 42357 (2017). https://doi.org/10.1038/srep42357

94. Y.F. Ma, S.C. Zhang, S.J. Din, X.X. Liu, X. Yu et al., Passively Q-switched Nd:GdLaNbO 4 laser based on 2D PdSe nanosheet. Opt. Laser Technol. 124(1), 105959 (2020). https://doi.org/10.1016/j.optlastec.2019.105959

95. Y.F. Ma, Z.F. Peng, S.J. Ding, H.Y. Sun, F. Peng et al., Twodimensional $\mathrm{WS}_{2}$ nanosheet based passively Q-switched Nd:GdLaNbO 4 laser. Opt. Laser Technnol. 115(1), 104-108 (2019). https://doi.org/10.1016/j.optlastec.2019.02.015

96. P.K. Cheng, C.Y. Tang, S. Ahmed, J. Qiao, L.H. Zeng et al., Utilization of group 10 2D TMDs-PdSe2 as a nonlinear optical material for obtaining switchable laser pulse generation modes. Nanotechnology 32(5), 055201 (2021). https://doi. org/10.1088/1361-6528/abc1a2

97. J. Pang, A. Bachmatiuk, Y. Yin, B. Trzebicka, L. Zhao et al., Applications of phosphorene and black phosphorus in energy conversion and storage devices. Adv. Energy Mater. 8(8), 1702093 (2018). https://doi.org/10.1002/aenm.201702093

98. J. Pang, R.G. Mendes, A. Bachmatiuk, L. Zhao, H.Q. Ta et al., Applications of 2D MXenes in energy conversion and storage systems. Chem. Soc. Rev. 48(1), 72-133 (2019). https://doi.org/10.1039/c8cs00324f

99. K. Olszowska, J. Pang, P.S. Wrobel, L. Zhao, H.Q. Ta et al., Three-dimensional nanostructured graphene: synthesis and energy, environmental and biomedical applications. Synth. Met. 234(1), 53-85 (2017). https://doi.org/10.1016/j.synth met.2017.10.014

100. J. Zhou, H. Chen, X. Zhang, K. Chi, Y. Cai et al., Substrate dependence on $\left(\mathrm{Sb}_{4} \mathrm{Se}_{6}\right)$ n ribbon orientations of antimony selenide thin films: morphology, carrier transport and photovoltaic performance. J. Alloys Compd. 862(1), 158703 (2021). https://doi.org/10.1016/j.jallcom.2021.158703

101. F. Shu, M. Wang, J. Pang, P. Yu, A free-standing superhydrophobic film for highly efficient removal of water from turbine oil. Front. Chem. Sci. Eng. 13(2), 393-399 (2019). https:// doi.org/10.1007/s11705-018-1754-3 
102. K. Wang, J. Pang, L. Li, S. Zhou, Y. Li et al., Synthesis of hydrophobic carbon nanotubes/reduced graphene oxide composite films by flash light irradiation. Front. Chem. Sci. Eng. 12(3), 376-382 (2018). https://doi.org/10.1007/ s11705-018-1705-z

103. Y. Yin, J. Pang, J. Wang, X. Lu, Q. Hao et al., Grapheneactivated optoplasmonic nanomembrane cavities for photodegradation detection. ACS Appl. Mater. Interfaces 11(17), 15891-15897 (2019). https://doi.org/10.1021/acsami.9b007 33

104. F.-X. Liang, J.-Z. Wang, Z.-X. Zhang, Y.-Y. Wang, Y. Gao et al., Broadband, ultrafast, self-driven photodetector based on Cs-doped $\mathrm{FAPbI}_{3}$ perovskite thin film. Adv. Opt. Mater. 5(22), 1700654 (2017). https://doi.org/10.1002/adom.20170 0654

105. M. Long, A. Gao, P. Wang, H. Xia, C. Ott et al., Room temperature high-detectivity mid-infrared photodetectors based on black arsenic phosphorus. Sci. Adv. 3(6), e1700589 (2017). https://doi.org/10.1126/sciadv.1700589

106. X. Yu, P. Yu, D. Wu, B. Singh, Q. Zeng et al., Atomically thin noble metal dichalcogenide: a broadband mid-infrared semiconductor. Nat. Commun. 9(1), 1545 (2018). https://doi. org/10.1038/s41467-018-03935-0

107. A.L. Hsu, P.K. Herring, N.M. Gabor, S. Ha, Y.C. Shin et al., Graphene-based thermopile for thermal imaging applications. Nano Lett. 15(11), 7211-7216 (2015). https://doi.org/ 10.1021/acs.nanolett.5b01755

108. J. Piotrowski, A. Rogalski, Uncooled long wavelength infrared photon detectors. Infrared Phys. Technol. 46(1-2), 115131 (2004). https://doi.org/10.1016/j.infrared.2004.03.016

109. Y. Cao, X. Zhu, H. Chen, X. Zhang, J. Zhouc et al., Towards high efficiency inverted $\mathrm{Sb}_{2} \mathrm{Se}_{3}$ thin film solar cells. Sol. Energy Mater. Sol. Cells 200(1), 109945 (2019). https://doi. org/10.1016/j.solmat.2019.109945

110. Y. Cao, X. Zhu, J. Jiang, C. Liu, J. Zhou et al., Rotational design of charge carrier transport layers for optimal antimony trisulfide solar cells and its integration in tandem devices. Sol. Energy Mater. Sol. Cells 206(1), 110279 (2020). https:// doi.org/10.1016/j.solmat.2019.110279

111. J. Jiang, F. Meng, Q. Cheng, A. Wang, Y. Chen et al., Low lattice mismatch InSe-Se vertical van der Waals heterostructure for high-performance transistors via strong fermi-level depinning. Small Methods 4(8), 2000238 (2020). https://doi. org/10.1002/smtd.202000238

112. J. Jiang, F. Meng, Q. Cheng, A. Wang, Y. Chen et al., Low lattice mismatch InSe-Se vertical van der Waals heterostructure for high-performance transistors via strong fermi-level depinning (Small Methods 8/2020). Small Methods 4(8), 2070032 (2020). https://doi.org/10.1002/smtd.202070032

113. C.-C. Wu, D. Jariwala, V.K. Sangwan, T.J. Marks, M.C. Hersam et al., Elucidating the photoresponse of ultrathin $\mathrm{MoS}_{2}$ field-effect transistors by scanning photocurrent microscopy. J. Phys. Chem. Lett. 4(15), 2508-2513 (2013). https://doi.org/ 10.1021/jz401199x

114. F. Xue, L. Chen, J. Chen, J. Liu, L. Wang et al., p-Type $\mathrm{MoS}_{2}$ and n-type $\mathrm{ZnO}$ diode and its performance enhancement by the piezophototronic effect. Adv. Mater. 28(17), 3391-3398 (2016). https://doi.org/10.1002/adma.201506472

115. D. Li, M. Chen, Z. Sun, P. Yu, Z. Liu et al., Two-dimensional non-volatile programmable $\mathrm{p}-\mathrm{n}$ junctions. Nat. Nanotechnol. 12(9), 901-906 (2017). https://doi.org/10.1038/nnano.2017. 104

116. X. Zhang, J. Grajal, J.L. Vazquez-Roy, U. Radhakrishna, $\mathrm{X}$. Wang et al., Two-dimensional $\mathrm{MoS}_{2}$-enabled flexible rectenna for Wi-Fi-band wireless energy harvesting. Nature 566(7744), 368-372 (2019). https://doi.org/10.1038/ s41586-019-0892-1

117. A.M. Afzal, G. Dastgeer, M.Z. Iqbal, P. Gautam, M.M. Faisal, High-performance p-BP/n-PdSe ${ }_{2}$ near-infrared photodiodes with a fast and gate-tunable photoresponse. ACS Appl. Mater. Interfaces 12(17), 19625-19634 (2020). https://doi.org/10. 1021/acsami.9b22898

118. J.A. Leñero-Bardallo, R. Carmona-Galán, A. RodríguezVázquez, Applications of event-based image sensors-review and analysis. Int. J. Circ. Theor. Appl. 46(9), 1620-1630 (2018). https://doi.org/10.1002/cta.2546

119. F.X. Liang, X.Y. Zhao, J.J. Jiang, J.G. Hu, W.Q. Xie et al., Light confinement effect induced highly sensitive, self-driven near-infrared photodetector and image sensor based on multilayer $\mathrm{PdSe}_{2}$ /pyramid Si heterojunction. Small 15(44), 1903831 (2019). https://doi.org/10.1002/smll.201903831

120. I. Ibrahim, J. Kalbacova, V. Engemaier, J.B. Pang, R.D. Rodriguez et al., Confirming the dual role of etchants during the enrichment of semiconducting single wall carbon nanotubes by chemical vapor deposition. Chem. Mater. 27(17), 59645973 (2015). https://doi.org/10.1021/acs.chemmater.5b02037

121. J. Pang, R.G. Mendes, P.S. Wrobel, M.D. Wlodarski, H.Q. Ta et al., Self-terminating confinement approach for large-area uniform monolayer graphene directly over $\mathrm{Si} / \mathrm{SiOx}$ by chemical vapor deposition. ACS Nano 11(2), 1946-1956 (2017). https://doi.org/10.1021/acsnano.6b08069

122. J. Pang, A. Bachmatiuk, I. Ibrahim, L. Fu, D. Placha et al., CVD growth of $1 \mathrm{D}$ and $2 \mathrm{D} \mathrm{sp}^{2}$ carbon nanomaterials. J. Mater. Sci. 51(2), 640-667 (2015). https://doi.org/10.1007/ s10853-015-9440-Z

123. A. Soni, L. Zhao, H.Q. Ta, Q. Shi, J. Pang et al., Facile graphitization of silicon nano-particles with ethanol based chemical vapor deposition. Nano-Struct. Nano-Objects 16(1), 38-44 (2018). https://doi.org/10.1016/j.nanoso.2018.04.001

124. B. Sun, J. Pang, Q. Cheng, S. Zhang, C. Zhang et al., Synthesis of wafer-scale graphene with chemical vapor deposition for electronic device applications. Adv. Mater. Technol. 1, 2000744 (2021). https://doi.org/10.1002/admt.202000744

125. G.S. Martynkova, F. Becerik, D. Placha, J. Pang, H. Akbulut et al., Effect of milling and annealing on carbon-silver system. J. Nanosci. Nanotechnol. 19(5), 2770-2774 (2019). https://doi.org/10.1166/jnn.2019.15869

126. M.H. Rummeli, S. Gorantla, A. Bachmatiuk, J. Phieler, N. Geissler et al., On the role of vapor trapping for chemical vapor deposition (CVD) grown graphene over copper. Chem. Mater. 25(24), 4861-4866 (2013). https://doi.org/ $10.1021 / \mathrm{cm} 401669 \mathrm{k}$ 
127. J.B. Pang, A. Bachmatiuk, L. Fu, C.L. Yan, M.Q. Zeng et al., Oxidation as a means to remove surface contaminants on $\mathrm{Cu}$ foil prior to graphene growth by chemical vapor deposition. J. Phys. Chem. C 119(23), 13363-13368 (2015). https://doi.org/10.1021/acs.jpcc.5b03911

128. J.B. Pang, A. Bachmatiuk, L. Fu, R.G. Mendes, M. Libera et al., Direct synthesis of graphene from adsorbed organic solvent molecules over copper. RSC Adv. 5(75), 6088460891 (2015). https://doi.org/10.1039/c5ra09405d

129. N.M. Santhosh, G. Filipič, E. Kovacevic, A. Jagodar, J. Berndt et al., $\mathrm{N}$-graphene nanowalls via plasma nitrogen incorporation and substitution: the experimental evidence. Nano-Micro Lett. 12(1), 53 (2020). https://doi.org/10.1007/ s40820-020-0395-5

130. R.G. Mendes, J. Pang, A. Bachmatiuk, H.Q. Ta, L. Zhao et al., Electron-driven in situ transmission electron microscopy of 2D transition metal dichalcogenides and their 2D heterostructures. ACS Nano 13(2), 978-995 (2019). https:// doi.org/10.1021/acsnano.8b08079

131. D. Zhang, T. Liu, J. Cheng, Q. Cao, G. Zheng et al., Lightweight and high-performance microwave absorber based on 2D WS - RGO heterostructures. Nano-Micro Lett. 11(1), 38 (2019). https://doi.org/10.1007/s40820-019-0270-4

132. K. Persson, Materials Data on $\mathrm{PdSe}_{2}$ (SG:61) by Materials Project. https://doi.org/10.17188/1199960

133. L.-Y. Feng, R.A.B. Villaos, Z.-Q. Huang, C.-H. Hsu, F.-C. Chuang, Layer-dependent band engineering of Pd dichalcogenides: a first-principles study. New J. Phys. 22(5), 053010 (2020). https://doi.org/10.1088/1367-2630/ab7d7a

134. K. Persson, Materials Data on $\mathrm{PdS}_{2}$ (SG:61) by Materials Project. https://doi.org/10.17188/1189716

135. K. Persson. Materials Data on $\mathrm{Te}_{2} \mathrm{Pd}$ (SG:164) by Materials Project. https://doi.org/10.17188/1307608

136. G. Anemone, P. Casado Aguilar, M. Garnica, F. Calleja, A. Al Taleb et al., Electron-phonon coupling in superconduct-

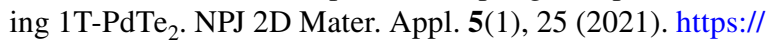
doi.org/10.1038/s41699-021-00204-5

137. R.N. Madhu, Singh, Palladium selenides as active methanol tolerant cathode materials for direct methanol fuel cell. Int. J. Hydrogen Energy 36(16), 10006-10012 (2011). https:// doi.org/10.1016/j.ijhydene.2011.05.069

138. D. Qin, P. Yan, G. Ding, X. Ge, H. Song et al., Monolayer $\mathrm{PdSe}_{2}$ : a promising two-dimensional thermoelectric material. Sci. Rep. 8(1), 2764 (2018). https://doi.org/10.1038/ s41598-018-20918-9

139. G. Zhang, M. Amani, A. Chaturvedi, C. Tan, J. Bullock et al., Optical and electrical properties of two-dimensional palladium diselenide. Appl. Phys. Lett. 114(25), 253102 (2019). https://doi.org/10.1063/1.5097825

140. A.N. Hoffman, Y. Gu, L. Liang, J.D. Fowlkes, K. Xiao et al., Exploring the air stability of $\mathrm{PdSe}_{2}$ via electrical transport measurements and defect calculations. NPJ 2D Mater. Appl. 3(1), 50 (2019). https://doi.org/10.1038/s41699-019-0132-4
141. H. Fang, W. Hu, Photogating in low dimensional photodetectors. Adv. Sci. 4(12), 1700323 (2017). https://doi.org/10. 1002/advs.201700323

142. P. Miro, M. Ghorbani-Asl, T. Heine, Two dimensional materials beyond $\mathrm{MoS}_{2}$ : noble-transition-metal dichalcogenides. Angew. Chem. Int. Ed. 53(11), 3015-3018 (2014). https:// doi.org/10.1002/anie.201309280

143. L. Li, W. Wang, Y. Chai, H. Li, M. Tian et al., Few-layered $\mathrm{PtS}_{2}$ phototransistor on h-BN with high gain. Adv. Funct. Mater. 27(27), 1701011 (2017). https://doi.org/10.1002/adfm. 201701011

144. H. Xu, H.P. Huang, H. Fei, J. Feng, H.R. Fuh et al., Strategy for fabricating wafer-scale platinum disulfide. ACS Appl. Mater. Interfaces 11(8), 8202-8209 (2019). https://doi.org/ 10.1021/acsami.8b19218

145. E. Zhang, Y. Jin, X. Yuan, W. Wang, C. Zhang et al., $\mathrm{ReS}_{2}$-based field-effect transistors and photodetectors. Adv. Funct. Mater. 25(26), 4076-4082 (2015). https://doi.org/10. 1002/adfm.201500969

146. J. Shim, A. Oh, D.H. Kang, S. Oh, S.K. Jang et al., High-performance $2 \mathrm{D}$ rhenium disulfide (ReS2) transistors and photodetectors by oxygen plasma treatment. Adv. Mater. 28(32), 6985-6992 (2016). https://doi.org/10.1002/adma.201601002

147. E. Zhang, P. Wang, Z. Li, H. Wang, C. Song et al., Tunable ambipolar polarization-sensitive photodetectors based on high-anisotropy $\mathrm{ReSe}_{2}$ nanosheets. ACS Nano 10(8), 80678077 (2016). https://doi.org/10.1021/acsnano.6b04165

148. M. Hafeez, L. Gan, H. Li, Y. Ma, T. Zhai, Chemical vapor deposition synthesis of ultrathin hexagonal $\mathrm{ReSe}_{2}$ flakes for anisotropic raman property and optoelectronic application. Adv. Mater. 28(37), 8296-8301 (2016). https://doi.org/10. 1002/adma.201601977

149. W. Feng, J.-B. Wu, X. Li, W. Zheng, X. Zhou et al., Ultrahigh photo-responsivity and detectivity in multilayer InSe nanosheets phototransistors with broadband response. J. Mater. Chem. C 3(27), 7022-7028 (2015). https://doi.org/ $10.1039 / \mathrm{c} 5 \mathrm{tc} 01208 \mathrm{~b}$

150. M. Dai, H. Chen, R. Feng, W. Feng, Y. Hu et al., A dual-band multilayer InSe self-powered photodetector with high performance induced by surface plasmon resonance and asymmetric Schottky junction. ACS Nano 12(8), 8739-8747 (2018). https://doi.org/10.1021/acsnano.8b04931

151. J. Ye, S. Soeda, Y. Nakamura, O. Nittono, Crystal structures and phase transformation in $\mathrm{In}_{2} \mathrm{Se}_{3}$ compound semiconductor. Jpn. J. Appl. Phys. 37, 4264-4271 (1998). https://doi.org/10. 1143/jjap.37.4264

152. W. Feng, F. Gao, Y. Hu, M. Dai, H. Liu et al., Phase-engineering-driven enhanced electronic and optoelectronic performance of multilayer In2Se3 nanosheets. ACS Appl. Mater. Interfaces 10(33), 27584-27588 (2018). https://doi.org/10. 1021/acsami.8b10194

153. R.B. Jacobs-Gedrim, M. Shanmugam, N. Jain, C.A. Durcan, M.T. Murphy et al., Extraordinary photoresponse in twodimensional $\operatorname{In}(2) \operatorname{Se}(3)$ nanosheets. ACS Nano 8(1), 514-521 (2014). https://doi.org/10.1021/nn405037s 
154. M. Amani, E. Regan, J. Bullock, G.H. Ahn, A. Javey, Midwave infrared photoconductors based on black phosphorusarsenic alloys. ACS Nano 11(11), 11724-11731 (2017). https://doi.org/10.1021/acsnano.7b07028

155. D. Zheng, H. Fang, M. Long, F. Wu, P. Wang et al., High-performance near-infrared photodetectors based on p-type SnX $(\mathrm{X}=\mathrm{S}, \mathrm{Se}$ ) nanowires grown via chemical vapor deposition. ACS Nano 12(7), 7239-7245 (2018). https://doi.org/10.1021/ acsnano.8b03291

156. G. Su, V.G. Hadjiev, P.E. Loya, J. Zhang, S. Lei et al., Chemical vapor deposition of thin crystals of layered semiconductor $\mathrm{SnS}_{2}$ for fast photodetection application. Nano Lett. 15(1), 506-513 (2015). https://doi.org/10.1021/nl503857r

157. F. Xia, T. Mueller, Y.M. Lin, A. Valdes-Garcia, P. Avouris, Ultrafast graphene photodetector. Nat. Nanotechnol. 4(12), 839-843 (2009). https://doi.org/10.1038/nnano.2009.292

158. B.J. Kim, H. Jang, S.K. Lee, B.H. Hong, J.H. Ahn et al., High-performance flexible graphene field effect transistors with ion gel gate dielectrics. Nano Lett. 10(9), 3464-3466 (2010). https://doi.org/10.1021/nl101559n

159. E.O. Polat, G. Mercier, I. Nikitskiy, E. Puma, T. Galan et al., Flexible graphene photodetectors for wearable fitness monitoring. Sci. Adv. 5(9), eaaw7846 (2019). https://doi.org/10. 1126/sciadv.aaw7846

160. X. Yu, Z. Dong, Y. Liu, T. Liu, J. Tao et al., A high performance, visible to mid-infrared photodetector based on graphene nanoribbons passivated with $\mathrm{HfO}_{2}$. Nanoscale 8(1), 327-332 (2016). https://doi.org/10.1039/c5nr06869j

161. L. Zeng, L. Tao, C. Tang, B. Zhou, H. Long et al., Highresponsivity UV-Vis photodetector based on transferable $\mathrm{WS}_{2}$ film deposited by magnetron sputtering. Sci. Rep. 6(1), 20343 (2016). https://doi.org/10.1038/srep20343

162. J. Jiang, Q. Zhang, A. Wang, Y. Zhang, F. Meng, C. Zhang, X. Feng, Y. Feng, L. Gu, H. Liu, L. Han, A facile and effective method for patching sulfur vacancies of WS2 via nitrogen plasma treatment. Small 15(36), 1901791 (2019). https://doi. org/10.1002/smll.201901791

163. Q. Wang, Q. Zhang, X. Zhao, Y.J. Zheng, J. Wang et al., High-energy gain upconversion in monolayer tungsten disulfide photodetectors. Nano Lett. 19(8), 5595-5603 (2019). https://doi.org/10.1021/acs.nanolett.9b02136

164. W. Zhang, M.H. Chiu, C.H. Chen, W. Chen, L.J. Li et al., Role of metal contacts in high-performance phototransistors based on $\mathrm{WSe}_{2}$ monolayers. ACS Nano 8(8), 8653-8661 (2014). https://doi.org/10.1021/nn503521c

165. H. Zhou, C. Wang, J.C. Shaw, R. Cheng, Y. Chen et al., Large area growth and electrical properties of p-type $\mathrm{WSe}_{2}$ atomic layers. Nano Lett. 15(1), 709-713 (2015). https://doi.org/10. 1021/n1504256y

166. J. Chen, Q. Wang, Y. Sheng, G. Cao, P. Yang et al., High-performance $\mathrm{WSe}_{2}$ photodetector based on a laser-induced $\mathrm{p}-\mathrm{n}$ junction. ACS Appl. Mater. Interfaces 11(46), 43330-43336 (2019). https://doi.org/10.1021/acsami.9b13948

167. H.S. Lee, S.W. Min, Y.G. Chang, M.K. Park, T. Nam et al., $\mathrm{MoS}(2)$ nanosheet phototransistors with thickness-modulated optical energy gap. Nano Lett. 12(7), 3695-3700 (2012). https://doi.org/10.1021/nl301485q

168. Y.H. Zhou, H.N. An, C. Gao, Z.Q. Zheng, B. Wang, UV-VisNIR photodetector based on monolayer $\mathrm{MoS}_{2}$. Mater. Lett. 237(1), 298-302 (2019). https://doi.org/10.1016/j.matlet. 2018.11.112

169. W. Wang, A. Klots, D. Prasai, Y. Yang, K.I. Bolotin et al., Hot electron-based near-infrared photodetection using bilayer $\mathrm{MoS}_{2}$. Nano Lett. 15(11), 7440-7444 (2015). https://doi.org/ 10.1021/acs.nanolett.5b02866

170. C. Jung, S.M. Kim, H. Moon, G. Han, J. Kwon et al., Highly crystalline CVD-grown multilayer $\mathrm{MoSe}_{2}$ thin film transistor for fast photodetector. Sci. Rep. 5(1), 15313 (2015). https:// doi.org/10.1038/srep15313

171. R. Coehoorn, C. Haas, R.A. de Groot, Electronic structure of $\mathrm{MoSe}_{2}, \mathrm{MoS}_{2}$, and $\mathrm{WSe}_{2}$. II. The nature of the optical band gaps. Phys. Rev. B 35(12), 6203-6206 (1987). https://doi.org/ 10.1103/physrevb.35.6203

172. P.J. Ko, A. Abderrahmane, N.H. Kim, A. Sandhu, Highperformance near-infrared photodetector based on nano-layered $\mathrm{MoSe}_{2}$. Semicond. Sci. Technol. 32(6), 065015 (2017). https://doi.org/10.1088/1361-6641/aa6819

173. V. Tran, R. Soklaski, Y. Liang, L. Yang, Layer-controlled band gap and anisotropic excitons in few-layer black phosphorus. Phys. Rev. B 89(23), 235319 (2014). https://doi.org/ 10.1103/PhysRevB.89.235319

174. Q. Guo, A. Pospischil, M. Bhuiyan, H. Jiang, H. Tian et al., Black phosphorus mid-infrared photodetectors with high gain. Nano Lett. 16(7), 4648-4655 (2016). https://doi.org/ 10.1021/acs.nanolett.6b01977

175. J. Qiao, X. Kong, Z.X. Hu, F. Yang, W. Ji, High-mobility transport anisotropy and linear dichroism in few-layer black phosphorus. Nat. Commun. 5(1), 4475 (2014). https://doi. org/10.1038/ncomms5475

176. J. Wang, A. Rousseau, E. Eizner, A.-L. Phaneuf-L'Heureux, L. Schue et al., Spectral responsivity and photoconductive gain in thin film black phosphorus photodetectors. ACS Photon. 6(12), 3092-3099 (2019). https://doi.org/10.1021/acsph otonics.9b00951

177. X. Zhou, X. Hu, B. Jin, J. Yu, K. Liu et al., Highly anisotropic GeSe nanosheets for phototransistors with ultrahigh photoresponsivity. Adv. Sci. 5(8), 1800478 (2018). https://doi.org/10. 1002/advs.201800478

178. C. Jia, D. Wu, E.P. Wu, J.W. Guo, Z.H. Zhao et al., A selfpowered high-performance photodetector based on a $\mathrm{MoS}_{2} /$ GaAs heterojunction with high polarization sensitivity. J. Mater. Chem. C. 7(13), 3817-3821 (2019). https://doi.org/ $10.1039 / \mathrm{c} 8 \mathrm{tc} 06398 \mathrm{~b}$

179. R. Chai, Y. Chen, M. Zhong, H. Yang, F. Yan et al., Nonlayered $\mathrm{ZnSb}$ nanoplates for room temperature infrared polarized photodetectors. J. Mater. Chem. C 8(19), 63886395 (2020). https://doi.org/10.1039/d0tc00755b

180. S. Deng, M.L. Tao, J. Mei, M. Li, Y. Zhang et al., Optical and piezoelectric properties of strained orthorhombic $\mathrm{PdS}_{2}$. IEEE Trans. Nanotechnol. 18(1), 358-364 (2019). https:// doi.org/10.1109/Tnano.2019.2908221 
181. Y. Deng, Z. Luo, N.J. Conrad, H. Liu, Y. Gong et al., Black phosphorus-monolayer $\mathrm{MoS}_{2}$ van der Waals heterojunction p-n diode. ACS Nano 8(8), 8292-8299 (2014). https://doi. org/10.1021/nn5027388

182. F. Yan, L. Zhao, A. Patane, P. Hu, X. Wei et al., Fast, multicolor photodetection with graphene-contacted $\mathrm{p}-\mathrm{GaSe} / \mathrm{n}$ InSe van der Waals heterostructures. Nanotechnology 28(27), 27LT01 (2017). https://doi.org/10.1088/1361-6528/ aa749e

183. X. Chen, H. Chen, Z. Wang, Y. Shan, D.W. Zhang et al., Analysis of the relationship between the contact barrier and rectification ratio in a two-dimensional $\mathrm{P}-\mathrm{N}$ heterojunction. Semicond. Sci. Technol. 33(11), 114012 (2018). https://doi. org/10.1088/1361-6641/aae3aa

184. K. Murali, M. Dandu, S. Das, K. Majumdar, Gate-tunable $\mathrm{WSe}_{2} / \mathrm{SnSe}_{2}$ backward diode with ultrahigh-reverse rectification ratio. ACS Appl. Mater. Interfaces 10(6), 5657-5664 (2018). https://doi.org/10.1021/acsami.7b18242

185. M.A. Khan, S. Rathi, D. Lim, S.J. Yun, D.-H. Youn et al., Gate tunable self-biased diode based on few layered $\mathrm{MoS}_{2}$ and $\mathrm{WSe}_{2}$. Chem. Mater. 30(3), 1011-1016 (2018). https:// doi.org/10.1021/acs.chemmater.7b04865

186. Z. Yang, L. Liao, F. Gong, F. Wang, Z. Wang et al., WSe $\mathrm{W}_{2} /$ GeSe heterojunction photodiode with giant gate tunability. Nano Energy 49(1), 103-108 (2018). https://doi.org/10. 1016/j.nanoen.2018.04.034

187. C. Lan, C. Li, S. Wang, T. He, T. Jiao et al., Zener tunneling and photoresponse of a $\mathrm{WS}_{2} / \mathrm{Si}$ van der Waals heterojunction. ACS Appl. Mater. Interfaces 8(28), 18375-18382 (2016). https://doi.org/10.1021/acsami.6b05109

188. J. Chu, F. Wang, L. Yin, L. Lei, C. Yan et al., High-performance ultraviolet photodetector based on a few-layered 2D $\mathrm{NiPS}_{3}$ nanosheet. Adv. Funct. Mater. 27(32), 1701342 (2017). https://doi.org/10.1002/adfm.201701342

189. L. Ye, H. Li, Z. Chen, J. Xu, Near-infrared photodetector based on $\mathrm{MoS}_{2}$ /black phosphorus heterojunction. ACS Photon. 3(4), 692-699 (2016). https://doi.org/10.1021/acsphotoni cs.6b00079

190. Y. Zhang, Y. Yu, L. Mi, H. Wang, Z. Zhu et al., In situ fabrication of vertical multilayered $\mathrm{MoS}_{2} / \mathrm{Si}$ homotype heterojunction for high-speed visible-near-infrared photodetectors.
Small 12(8), 1062-1071 (2016). https://doi.org/10.1002/smll. 201502923

191. Q. Liu, B. Cook, M. Gong, Y. Gong, D. Ewing et al., Printable transfer-free and wafer-size $\mathrm{MoS}_{2}$ /graphene van der Waals heterostructures for high-performance photodetection. ACS Appl. Mater. Interfaces 9(14), 12728-12733 (2017). https:// doi.org/10.1021/acsami.7b00912

192. A. Gundimeda, S. Krishna, N. Aggarwal, A. Sharma, N.D. Sharma et al., Fabrication of non-polar GaN based highly responsive and fast UV photodetector. Appl. Phys. Lett. 110(10), 103507 (2017). https://doi.org/10.1063/1.4978427

193. P. Wang, S. Liu, W. Luo, H. Fang, F. Gong et al., Arrayed van der Waals broadband detectors for dual-band detection. Adv. Mater. 29(16), 1521-4095 (2017). https://doi.org/10.1002/ adma.201604439

194. D.S. Um, Y. Lee, S. Lim, J. Park, W.C. Yen et al., InGaAs nanomembrane/si van der waals heterojunction photodiodes with broadband and high photoresponsivity. ACS Appl. Mater. Interfaces 8(39), 26105-26111 (2016). https://doi. org/10.1021/acsami.6b06580

195. W. Zheng, R. Lin, Y. Zhu, Z. Zhang, X. Ji et al., Vacuum ultraviolet photodetection in two-dimensional oxides. ACS Appl. Mater. Interfaces 10(24), 20696-20702 (2018). https:// doi.org/10.1021/acsami.8b04866

196. L.H. Zeng, M.Z. Wang, H. Hu, B. Nie, Y.Q. Yu et al., Monolayer graphene/germanium Schottky junction as high-performance self-driven infrared light photodetector. ACS Appl. Mater. Interfaces 5(19), 9362-9366 (2013). https://doi.org/ 10.1021/am4026505

197. X. Li, M. Zhu, M. Du, Z. Lv, L. Zhang et al., High detectivity graphene-silicon heterojunction photodetector. Small 12(5), 595-601 (2016). https://doi.org/10.1002/smll.201502336

198. K. Zhang, X. Fang, Y. Wang, Y. Wan, Q. Song et al., Ultrasensitive near-infrared photodetectors based on a graphene$\mathrm{MoTe}_{2}$-graphene vertical van der Waals heterostructure. ACS Appl. Mater. Interfaces 9(6), 5392-5398 (2017). https://doi. org/10.1021/acsami.6b14483

199. Y.-S. Lan, X.-R. Chen, C.-E. Hu, Y. Cheng, Q.-F. Chen, Penta-PdX $\mathrm{X}_{2}(\mathrm{X}=\mathrm{S}, \mathrm{Se}, \mathrm{Te})$ monolayers: promising anisotropic thermoelectric materials. J. Mater. Chem. A 7(18), 11134-11142 (2019). https://doi.org/10.1039/c9ta02138h 